\title{
Ramond-Ramond Fields, Fractional Branes and Orbifold Differential K-Theory
}

\author{
Richard J. Szabo ${ }^{1}$, Alessandro Valentino ${ }^{2}$ \\ 1 Department of Mathematics and Maxwell Institute for Mathematical Sciences, \\ Heriot-Watt University, Colin Maclaurin Building, Riccarton, \\ Edinburgh EH14 4AS, U.K. E-mail: R.J.Szabo@ma.hw.ac.uk \\ 2 Courant Research Center, "Higher Order Structures" and Mathematisches Institut, \\ Georg-August-Universität Göttingen, Bunsenstr. 3-5, \\ D-37073 Göttingen, Germany. E-mail: sandro@uni-math.gwdg.de
}

Received: 28 January 2008 / Accepted: 10 November 2009

Published online: 25 December 2009 - C Springer-Verlag 2009

\begin{abstract}
We study D-branes and Ramond-Ramond fields on global orbifolds of Type II string theory with vanishing $H$-flux using methods of equivariant K-theory and K-homology. We illustrate how Bredon equivariant cohomology naturally realizes stringy orbifold cohomology. We emphasize its role as the correct cohomological tool which captures known features of the low-energy effective field theory, and which provides new consistency conditions for fractional D-branes and Ramond-Ramond fields on orbifolds. We use an equivariant Chern character from equivariant K-theory to Bredon cohomology to define new Ramond-Ramond couplings of D-branes which generalize previous examples. We propose a definition for groups of differential characters associated to equivariant K-theory. We derive a Dirac quantization rule for Ramond-Ramond fluxes, and study flat Ramond-Ramond potentials on orbifolds.
\end{abstract}

\section{Introduction}

The study of fluxes and D-branes has been of fundamental importance in understanding the nonperturbative structures of string theory and M-theory. It has also established a common ground on which a fruitful interaction between physics and mathematics takes place. For example, the seminal papers $[49,62]$ demonstrated that D-brane charges in Type II superstring theory are classified by the K-theory of the spacetime manifold, and that ordinary cohomology alone cannot account for certain physical features induced by the dynamics of D-branes. As emphasized by refs. [2,36,55,60], and analyzed in great detail in refs. [56,57], another description of D-branes is provided by K-homology which sheds light on their geometrical nature and suggests that the standard picture of a D-brane as a submanifold of spacetime equipped with a vector bundle (and connection) should be modified.

Ramond-Ramond fields are dual objects to D-branes and have also been extensively investigated, but until recently their geometric nature has remained somewhat obscure. In ref. [52] it was proposed that Ramond-Ramond fields are also classified by K-theory, 
and that their total field strengths lie in the image of the Chern character homomorphism from K-theory to ordinary cohomology. This result led to the understanding that the Ramond-Ramond field is correctly understood as a self-dual field quantized by K-theory, and it explains various subtle issues surrounding the partition functions of these fields. In refs. [28,29] it was proposed that these properties are most naturally formulated by regarding Ramond-Ramond fields as cocycles for the differential K-theory of spacetime, an elegant description that allows one to study the gauge theory of Ramond-Ramond fields in topologically non-trivial backgrounds which naturally incorporates consistency conditions such as anomaly cancellation on branes in string theory and M-theory. These issues were among the motivations that led to the foundational paper [37], in which a detailed, elaborate construction for generalized differential cohomology theories is given. The importance of these mathematical theories has been greatly emphasized in refs. [32,33], where they are used to define and understand certain novel properties of quantum Hilbert spaces of abelian gauge field fluxes. A twisted version of differential K-theory has been proposed in refs. $[11,33]$ and applied to the quantization of Ramond-Ramond fields in an $H$-flux background, while a rigorous geometrical definition of this theory has been developed recently in ref. [18].

The goal of this paper is to extend these lines of developments to study properties of Ramond-Ramond fields and D-branes in orbifolds of Type II superstring theory with vanishing $H$-flux. We limit our study to the cases of good (or global) orbifolds $[X / G]$, where $X$ is a manifold and $G$ is a finite group acting via diffeomorphisms of $X$. It is possible to resolve singularities in the orbifold where it fails to be a manifold, and replace the quotient space by a non-compact manifold with appropriate asymptotic behaviour. However, orbifold singularities do not pose a problem and one can still have consistent superstrings propagating on orbifolds [24,25]. It was proposed in ref. [62] that D-branes on the orbifold spacetime $[X / G]$ are classified by the $G$-equivariant K-theory of the covering space $X$, as defined in ref. [58]. A recent overview of related developments in the case of abelian orbifolds can be found in ref. [41].

One of the main new ingredients that we introduce into the description of D-branes and fluxes on orbifolds is the use of Bredon cohomology [16,23]. This is a powerful equivariant cohomology theory that has both advantages and pitfalls. In contrast to the more commonly used Borel equivariant cohomology, Bredon cohomology is a good "approximation" to the classification of D-brane charges. We will support this statement by showing that it correctly captures the properties of Ramond-Ramond fields on an orbifold, in particular it naturally takes into account the twisted sectors of the string theory. It thereby gives a precise, rigorous realization of stringy orbifold cohomology. We will also see that it naturally arises in the Atiyah-Hirzebruch spectral sequence for equivariant K-theory, a fact that we shall exploit to describe new consistency conditions for D-branes and fluxes on orbifolds in terms of classes in the Bredon cohomology of the covering space $X$. Related to this feature is the fact that this equivariant cohomology theory is the target for a Chern character homomorphism on equivariant K-theory, defined in ref. [46], which induces an isomorphism when tensored over $\mathbb{R}$. By means of this technology, we present new compact and elegant expressions for the Wess-Zumino couplings of Ramond-Ramond fields to D-branes on $[X / G]$. This generalizes the usual Ramond-Ramond couplings [49] to orbifolds, and yields appropriate correction terms to previous flat space formulas. The major drawback of Bredon cohomology is that it is a rather difficult, abstract theory to define, and is even more difficult to explicitly calculate than other equivariant cohomology theories. 
Another main achievement of this paper is a proposed definition of differential K-theory suitable for orbifolds. Though extremely powerful and general, the machinery developed in ref. [37] cannot be immediately applied to an equivariant cohomology functor on the category of $G$-manifolds. By using Bredon cohomology and the equivariant Chern character, we define abelian groups that behave as natural generalizations of the ordinary differential K-theory groups, in the sense that they agree in the case of a trivial group and they satisfy analogous exact sequences. Although far from having the generality of the work of ref. [37], our construction gives a systematic framework in which to study Ramond-Ramond fields on orbifolds with a Dirac quantization condition, including non-trivial contributions from flat potentials, and it represents a first step in the development of generalized differential cohomology theories in the equivariant setting. It is here that the use of Bredon cohomology is particularly important, both because of the equivariant Chern character isomorphism and because the framework requires explicit use of differential forms, neither of which can be accommodated directly by the Borel construction.

The outline of the remainder of this paper is as follows. In Sect. 1 we summarize some basic notions about the cohomology theories of spaces with group actions. In Sect. 2 we present a detailed definition of Bredon cohomology and the construction of the equivariant Chern character of ref. [46], as these have not made appearances before in the physics literature. These first two sections give the main mathematical background for the rest of the paper. In Sect. 3 we make a brief excursion into the description of D-branes using geometric equivariant K-homology, showing that the use of K-cycles is very well-suited to the description of fractional D-branes and their topological charges computed using equivariant Dirac operator theory. In Sect. 4 we use Bredon cohomology and the equivariant Chern character to define Ramond-Ramond couplings to D-branes on orbifolds and compare it with previous examples in the literature. Our formulas include the appropriate gravitational contributions which are derived from an equivariant version of the Riemann-Roch theorem and equivariant index theory. In Sect. 5 we give a detailed mathematical construction of the orbifold differential $\mathrm{K}$-theory groups, and prove that they fit into appropriate exact sequences which are useful in applications. In Sect. 6 we use the orbifold differential K-theory to describe the flux quantization of Ramond-Ramond fields on orbifolds by writing an equivariant version of the Ramond-Ramond current in terms of the equivariant Chern character. We also study the group of flat potentials in detail, and illustrate how the spectral sequence for equivariant K-theory can be used to determine obstruction classes in Bredon cohomology which yield stability conditions for D-branes and fluxes on orbifolds. Appendix A contains some background material on functor categories used in the main text, Appendix $\mathrm{B}$ records the definitions of equivariant K-homology, while Appendix C demonstrates the use of geometric equivariant K-cycles in the classification of D-brane charges on orbifolds.

\section{Cohomology of Spaces with Symmetries}

In this section we will recall some basic notions about (generalized) equivariant cohomology theories that we will need throughout this paper. In the following, $X$ denotes a topological space and $G$ a finite group, unless otherwise stated. Throughout a (left) action $G \times X \rightarrow X$ of $G$ on $X$ will be denoted $(g, x) \mapsto g \cdot x$. The stabilizer or isotropy group of a point $x \in X$ is denoted $G_{x}=\{g \in G \mid g \cdot x=x\}$. Recall that a continuous map $f: X \rightarrow Y$ of $G$-spaces is a $G$-map if $f(g \cdot x)=g \cdot f(x)$ for all $g \in G$ and $x \in X$. 
1.1. $G$-complexes. A $G$-equivariant $C W$-decomposition of a $G$-space $X$ consists of a filtration $X_{n}, n \in \mathbb{N}_{0}$ such that

$$
X=\bigcup_{n \in \mathbb{N}_{0}} X_{n}
$$

and $X_{n}$ is obtained from $X_{n-1}$ by "attaching" equivariant cells by the following procedure. Define

$$
X_{0}=\coprod_{j \in J_{0}} G / K_{j},
$$

with $K_{j}$ a collection of subgroups of $G$ and the standard (left) $G$-action on any coset space $G / K_{j}$. For $n \geq 1$ set

$$
X_{n}=\left(X_{n-1} \amalg \coprod_{j \in J_{n}}\left(\mathbb{B}_{j}^{n} \times G / K_{j}\right)\right) / \sim,
$$

where the equivalence relation $\sim$ is generated by $G$-equivariant "attaching maps"

$$
\phi_{j}^{n}: \mathbb{S}_{j}^{n-1} \times G / K_{j} \longrightarrow X_{n-1} .
$$

One requires that $X$ carries the colimit topology with respect to $\left(X_{n}\right)$, i.e., $B \subset X$ is closed if and only if $B \cap X_{n}$ is closed in $X_{n}$ for all $n \in \mathbb{N}_{0}$. We call the image of $\mathbb{B}_{j}^{n} \times G / K_{j}$ (resp. $\stackrel{B}{B}_{j}^{n} \times G / K_{j}$ ) a closed (resp. open) $n$-cell of orbit type $G / K_{j}$. As usual, we call the subspace $X_{n}$ the $n$-skeleton of $X$. If $X=X_{n}$ and $X \neq X_{n-1}$, then $n$ is called the (cellular) dimension of $X$ and $X$ is said to be of finite type. A $G$-space with a $G$-equivariant $\mathrm{CW}$-decomposition is called a $G$-complex.

When $G=e$ is the trivial group, a $G$-complex is just an ordinary CW-complex. In general, if $X$ is a $G$-complex then the orbit space $X / G$ is an ordinary CW-complex. Conversely, there is an intimate relation between $G$-complexes and ordinary $\mathrm{CW}$ complexes whenever $G$ is a discrete group. Let $X$ be a $G$-space which is an ordinary CW-complex. We say that $G$ acts cellularly on $X$ if

1) For each $g \in G$ and each open cell $E$ of $X$, the left translation $g \cdot E$ is again an open cell of $X$; and

2) If $g \cdot E=E$, then the induced map $E \rightarrow E, x \mapsto g \cdot x$ is the identity.

Then we have the following

Proposition 1.1. Let $X$ be a $C W$-complex with a cellular action of a discrete group $G$. Then $X$ is a $G$-complex with $n$-skeleton $X_{n}$.

In the case that $X$ is a smooth manifold, we require the $G$-action on $X$ to be smooth and there is an analogous result. Recall that the applicability of algebraic topology to manifolds relies on the fact that any manifold comes equipped with a canonical $\mathrm{CW}$-decomposition. In the case in which a group acts on the manifold one has the following result due to Illman [38,39].

Theorem 1.2. If $G$ is a compact Lie group or a finite group acting on a smooth compact manifold $X$, then $X$ is triangulable as a finite $G$-complex.

The collection of $G$-complexes with $G$-maps as morphisms form a category. We are interested in equivariant cohomology theories defined on this category (or on subcategories thereof). 
1.2. Equivariant cohomology theories. We will now briefly spell out the main ingredients involved in building an equivariant cohomology theory on the category of finite $G$-complexes, leaving the details to the comprehensive treatments of refs. [23 and 45], and focusing instead on some explicit examples. Fix a group $G$ and a commutative ring $R$. A $G$-cohomology theory $\mathrm{E}_{G}^{\bullet}$ with values in $R$-modules is a collection of contravariant functors $\mathrm{E}_{G}^{n}$ from the category of $G$-CW pairs to the category of $R$-modules indexed by $n \in \mathbb{Z}$ together with natural transformations

$$
\delta_{G}^{n}(X, A): \mathrm{E}_{G}^{n}(X, A) \longrightarrow \mathrm{E}_{G}^{n+1}(X):=\mathrm{E}_{G}^{n+1}(X, \emptyset)
$$

for all $n \in \mathbb{Z}$ satisfying the axioms of $G$-homotopy invariance, long exact sequence of a pair, excision, and disjoint union. The theory is called ordinary if for any orbit $G / H$ one has $\mathrm{E}_{G}^{q}(G / H)=0$ for all $q \neq 0$. These axioms are formulated in an analogous way to that of ordinary cohomology. The new ingredient in an equivariant cohomology theory (which we have not yet defined) are the induction structures, which we shall now describe.

Let $\alpha: H \rightarrow G$ be a group homomorphism, and let $X$ be an $H$-space. Define the induction of $X$ with respect to $\alpha$ to be the $G$-space $\operatorname{ind}_{\alpha} X$ given by

$$
\operatorname{ind}_{\alpha} X:=G \times_{\alpha} X \text {. }
$$

This is the quotient of the product $G \times X$ by the $H$-action $h \cdot(g, x):=\left(g \alpha\left(h^{-1}\right), h \cdot x\right)$, with the $G$-action on $\operatorname{ind}_{\alpha} X$ given by $g^{\prime} \cdot[g, x]=\left[g^{\prime} g, x\right]$. If $H<G$ and $\alpha$ is the subgroup inclusion, the induced $G$-space is denoted $G \times{ }_{H} X$.

An equivariant cohomology theory $\mathrm{E}_{(-)}^{\bullet}$ with values in $R$-modules consists of a collection of $G$-cohomology theories $\mathrm{E}_{G}^{\bullet}$ with values in $R$-modules for each group $G$ such that for any group homomorphism $\alpha: H \rightarrow G$ and any $H$-CW pair $(X, A)$ with $\operatorname{ker}(\alpha)$ acting freely on $X$, there are for each $n \in \mathbb{Z}$ natural isomorphisms

$$
\operatorname{ind}_{\alpha}: \mathrm{E}_{G}^{n}\left(\operatorname{ind}_{\alpha}(X, A)\right) \stackrel{\approx}{\rightarrow} \mathrm{E}_{H}^{n}(X, A)
$$

satisfying

(a) Compatibility with the coboundary homomorphisms:

$$
\delta_{H}^{n} \circ \operatorname{ind}_{\alpha}=\operatorname{ind}_{\alpha} \circ \delta_{G}^{n}
$$

(b) Functoriality: If $\beta: G \rightarrow K$ is another group homomorphism such that $\operatorname{ker}(\beta \circ \alpha)$ acts freely on $X$, then for every $n \in \mathbb{Z}$ one has

$$
\operatorname{ind}_{\beta \circ \alpha}=\operatorname{ind}_{\alpha} \circ \operatorname{ind}_{\beta} \circ \mathrm{E}_{K}^{n}\left(f_{1}\right) \text {, }
$$

where

$$
\begin{aligned}
\left.f_{1}: \operatorname{ind}_{\beta} \operatorname{ind}_{\alpha}(X, A)\right) & \stackrel{\approx}{\rightarrow} \operatorname{ind}_{\beta \circ \alpha}(X, A) \\
(k, g, x) & \longmapsto(k \beta(g), x)
\end{aligned}
$$

is a $K$-homeomorphism and $\mathrm{E}_{K}^{n}\left(f_{1}\right)$ is the morphism on $K$-cohomology induced by $f_{1}$; and 
(c) Compatibility with conjugation: For $g, g^{\prime} \in G$ define $\operatorname{Ad}_{g}\left(g^{\prime}\right)=g g^{\prime} g^{-1}$. Then the homomorphism ind $\operatorname{Ad}_{g}$ coincides with $\mathrm{E}_{G}^{n}\left(f_{2}\right)$, where

$$
\begin{aligned}
f_{2}:(X, A) & \stackrel{\approx}{\rightarrow} \operatorname{ind}_{\mathrm{Ad}_{g}}(X, A) \\
x & \longmapsto\left(e, g^{-1} \cdot x\right)
\end{aligned}
$$

is a $G$-homeomorphism, where throughout $e$ denotes the identity element in the group $G$.

Thus the induction structures connect the various $G$-cohomologies and keep track of the equivariance. They will be very important in the construction of the equivariant Chern character for equivariant K-theory in the next section, even if we are only interested in a fixed group $G$.

Example 1.3 (Borel cohomology). Let $\mathrm{H}^{\bullet}$ be a cohomology theory for CW-pairs (for example, singular cohomology). Define

$$
\mathrm{H}_{G}^{n}(X, A):=\mathrm{H}^{n}\left(E G \times_{G}(X, A)\right),
$$

where $E G$ is the total space of the classifying principal $G$-bundle $E G \rightarrow B G$ which is contractible and carries a free $G$-action. This is called (equivariant) Borel cohomol$o g y$, and it is the most commonly used form of equivariant cohomology in the physics literature. Note that $\mathrm{H}_{G}^{\bullet}$ is well-defined because the quotient $E G \times_{G} X$ is unique up to the homotopy type of $X / G$. The ordinary $G$-cohomology structures on $\mathrm{H}_{G}^{\bullet}$ are inherited from the cohomology structures on $\mathrm{H}^{\bullet}$. The induction structures for $\mathrm{H}_{G}^{\bullet}$ are constructed as follows. Let $\alpha: H \rightarrow G$ be a group homomorphism and $X$ an $H$-space. Define

$$
\begin{aligned}
b: E H \times{ }_{H} X & \longrightarrow E G \times_{G} G \times_{\alpha} X, \\
(\varepsilon, x) & \longmapsto(E \alpha(\varepsilon), e, x),
\end{aligned}
$$

where $\varepsilon \in E H, x \in X$ and $E \alpha: E H \rightarrow E G$ is the $\alpha$-equivariant map induced by $\alpha$. The induction map ind ${ }_{\alpha}$ is then given by pullback

$$
\operatorname{ind}_{\alpha}:=b^{*}: \mathrm{H}_{G}^{n}\left(\operatorname{ind}_{\alpha} X\right)=\mathrm{H}^{n}\left(E G \times_{G} G \times_{\alpha} X\right) \longrightarrow \mathrm{H}^{n}\left(E H \times_{H} X\right)=\mathrm{H}_{H}^{n}(X) .
$$

If $\operatorname{ker}(\alpha)$ acts freely on $X$, then the map $b$ is a homotopy equivalence and hence the map ind $\mathrm{i}_{\alpha}$ is an isomorphism.

Example 1.4 (Equivariant $K$-theory). In ref. [58], equivariant topological K-theory is defined for any $G$-complex $X$ as the abelian group completion of the semigroup $\operatorname{Vect}_{G}^{\mathbb{C}}(X)$ of complex $G$-vector bundles over $X$, i.e., bundles $E \rightarrow X$ together with a lift of the $G$-action on $X$ to the fibres. The higher groups are defined via iterated suspension. To define the induction structures, recall that if $X$ is an $H$-space and $\alpha: H \rightarrow G$ is a group homomorphism, then the map

$$
\begin{aligned}
\varphi: X & \longrightarrow G \times_{\alpha} X \\
x & \longmapsto(e, x)
\end{aligned}
$$

is an $\alpha$-equivariant map which embeds $X$ as the subspace $H \times{ }_{\alpha} X$ of $G \times_{\alpha} X$, and which induces via pullback of vector bundles the homomorphism

$$
\varphi^{*}: \mathrm{K}_{G}^{\bullet}\left(G \times_{\alpha} X\right) \longrightarrow \mathrm{K}_{H}^{\bullet}(X) .
$$


This map defines the induction structure. It is invertible when $\operatorname{ker}(\alpha)$ acts freely on $X$, with inverse the "extension" map $E \mapsto G \times_{H} E$ for any $H$-vector bundle $E$ over $X$. The induction structure can be used to prove the well-known equivariant excision theorem

$$
\mathrm{K}_{G / N}^{\bullet}(X / N) \cong \mathrm{K}_{G}^{\bullet}(X),
$$

where $N$ is a normal subgroup of $G$ acting freely on $X$. Indeed, one has

$$
X / N \cong(G / N) \times_{G} X
$$

and if we define $\alpha: G \rightarrow G / N$ to be the quotient map, then

$$
\mathrm{K}_{G / N}^{\bullet}\left((G / N) \times_{\alpha} X\right) \cong \mathrm{K}_{G}^{\bullet}(X) .
$$

since $\operatorname{ker}(\alpha)=N$ acts freely on $X$.

\section{The Equivariant Chern Character}

In this section we will describe the equivariant Chern character for the $\mathrm{K}_{G}^{\bullet}$ functor and its target cohomology theory, Bredon cohomology. To this end, we will introduce some technology related to modules over functor categories, giving the necessary definitions and directing the reader to the relevant literature for further details. Some pertinent aspects of functor categories are summarized in Appendix A.

2.1. Chern character in topological K-theory. Let us begin by recalling some basic notions about the ordinary Chern character. Define $\pi_{-} \cdot \mathrm{K}$ to be the complex K-theory ring of the point. It is the $\mathbb{Z}$-graded ring $\mathbb{Z}\left[\left[u, u^{-1}\right]\right]$ of Laurent polynomials freely generated by an element $u$ of degree $\operatorname{deg}(u)=2$, where $u^{-1} \in \mathrm{K}^{-2}(\mathrm{pt})$ is called the Bott element and is represented by the Hopf bundle over $\mathbb{S}^{2}$. One then has a homomorphism

$$
\text { ch }: \mathrm{K}^{\bullet}(X) \longrightarrow \mathrm{H}\left(X ; \mathbb{R} \otimes \pi_{-\bullet} \mathrm{K}\right)^{\bullet}
$$

which induces the natural $\mathbb{Z}$-graded ring isomorphism

$$
\mathrm{K}^{\bullet}(X) \otimes \mathbb{R} \stackrel{\approx}{\rightarrow} \mathrm{H}\left(X ; \mathbb{R} \otimes \pi_{-\bullet} \mathrm{K}\right)^{\bullet}
$$

for any finite CW-complex $X$. This statement is true even if we tensor over $\mathbb{Q}$. The use here of the K-theory of the point as the coefficient ring serves just as a re-grading of the cohomology ring $\mathrm{H}^{\bullet}(X ; \mathbb{R})$. For example, it is easy to check that

$$
\mathrm{H}\left(X ; \mathbb{R} \otimes \pi_{-\bullet} \mathrm{K}\right)^{0} \cong \mathrm{H}^{\mathrm{even}}(X ; \mathbb{R}) .
$$

In particular, the Chern character tells us that K-theory and cohomology are the same thing up to torsion.

It is natural now to ask if there exists such a morphism for equivariant K-theory. One might naively think that the correct target theory for the equivariant Chern character would naturally be Borel cohomology. But the problem is much more subtle than it first may seem. The crucial point is that while in the ordinary cohomology of (finite) $\mathrm{CW}$-complexes the building blocks are the cohomology groups of a point, in the equivariant case they are the cohomology groups of the orbits $G / H$ for all subgroups $H$ 
of $G$, as we saw in Sect. 1.1. Any equivariant cohomology theory $\mathrm{E}_{G}^{\bullet}$ on the category of finite $G$-complexes is completely specified by its value on the orbit spaces $G / H$. A localization theorem due to Atiyah and Segal [4] tells us that the Borel cohomology of a $G$-space $X$ is isomorphic to its equivariant K-theory localized at the augmentation ideal in the representation ring $R(G)$ consisting of all elements whose characters vanish at the identity $e$ in $G$ (regarding $\mathrm{K}_{G}^{\bullet}(X)$ as a module over $R(G)$ ). Localizing at a prime ideal of $R(G)$ corresponds to restricting $X$ to the set of fixed points of an associated conjugacy class of cyclic subgroups of $G$. In this sense, Borel cohomology does not take into account the "contributions" of the non-trivial elements in $G$, and hence of the fixed points of the $G$-action.

There are several approaches to the equivariant Chern character (see refs. $[1,5,15$, $31,59]$, for example) which strongly depend on the types of groups involved (discrete, continuous, etc.) and on the ring one tensors with $(\mathbb{R}, \mathbb{C}$, etc.). As we are interested in finite groups and real coefficients for our physical applications later on, we will use the Chern character constructed in refs. [45 and 46]. Thus we proceed to the more abstract, but powerful and compact, definition of Bredon cohomology, which will turn out to be the best suited equivariant cohomology theory for all of our purposes.

2.2. Bredon cohomology. Let $G$ be a discrete group. The orbit category $\operatorname{Or}(G)$ of $G$ is defined as the category whose objects are homogeneous spaces $G / H$, with $H<G$, and whose morphisms are $G$-maps between them. From general considerations [23] it follows that a $G$-map between two homogeneous spaces $G / H$ and $G / K$ exists if and only if $H$ is conjugate to a subgroup of $K$, and hence any such map is of the form

$$
(g H \longmapsto g a K)
$$

for some $a \in G$ such that $a^{-1} H a<K$. If $\mathfrak{F}$ is any family of subgroups of $G$ then there is a subcategory $\operatorname{Or}(G, \mathfrak{F})$ with objects $G / H$ for $H \in \mathfrak{F}$. A simple example is provided by the cyclic groups $G=\mathbb{Z}_{p}$ with $p$ prime, for which the orbit category has just two objects, $G / e=G$ and $G / G=\mathrm{pt}$.

If $\mathrm{Ab}$ denotes the category of abelian groups, then a coefficient system is a functor

$$
\underline{F}: \operatorname{Or}(G)^{\mathrm{op}} \longrightarrow \mathrm{Ab}
$$

where $\operatorname{Or}(G)^{\text {op }}$ denotes the dual category to $\operatorname{Or}(G)$. With such a functor and any $G$-complex $X,{ }^{1}$ one can define for each $n \in \mathbb{Z}$ the group

$$
C_{G}^{n}(X, \underline{F}):=\operatorname{Hom}_{\mathrm{Or}(G)}\left(\underline{C}_{n}(X), \underline{F}\right),
$$

where $\underline{C}_{n}(X): \operatorname{Or}(G)^{\mathrm{op}} \rightarrow$ Ab is the projective functor defined by

$$
\underline{C}_{n}(X)(G / H):=C_{n}\left(X^{H}\right),
$$

the cellular homology of the fixed point complex

$$
X^{H}:=\{x \in X \mid h \cdot x=x \quad \forall h \in H\} .
$$

\footnotetext{
1 When $G$ is an infinite discrete group, one should restrict to proper $G$-complexes, i.e., with finite stabilizer for any point of $X$. Some further minor assumptions are needed when $G$ is a Lie group.
} 
In Eq. (2.2), $\operatorname{Hom}_{\mathrm{Or}(G)}(-,-)$ denotes the group of natural transformations between two contravariant functors, with the group structure inherited by the images of the functors in $A b$. The functoriality property of $\underline{C}_{n}(X)(G / H)$ is the natural one induced by the identification $X^{H} \cong \operatorname{Map}_{G}(G / H, X)$. Indeed, the two maps

$$
\begin{array}{ll}
X^{H} \longrightarrow \operatorname{Map}_{G}(G / H, X), & x \longmapsto f_{x}([g H])=g \cdot x, \\
\operatorname{Map}_{G}(G / H, X) \longrightarrow X^{H}, & f \longmapsto f(H)
\end{array}
$$

are easily seen to be inverse to each other, and the desired homeomorphism is obtained by giving the space $\operatorname{Map}_{G}(G / H, X)$ the compact-open topology. In particular, a $G$-map (2.1) induces a cellular map $X^{K} \rightarrow X^{H}, x \mapsto a \cdot x$.

These groups can be expressed in terms of the $G$-complex structure of $X$. If the $n$-skeleton $X_{n}$ is obtained by attaching equivariant cells as in Eq. (1.1) with $K_{j}$ the stabilizer of an $n$-cell of $X$, then the cellular chain complex $C_{\bullet}(X)$ consists of $G$-modules $C_{n}(X)=\bigoplus_{j \in J_{n}} \mathbb{Z}\left[G / K_{j}\right]$, and hence

$$
\underline{C}_{n}(X)(G / H) \cong \bigoplus_{j \in J_{n}} \mathbb{Z}\left[\operatorname{Mor} \operatorname{Or}_{(G)}\left(G / H, G / K_{j}\right)\right] .
$$

For each $n \geq 0$, the group $C_{G}^{n}(X, \underline{F})$ is the direct limit functor over all $n$-cells of orbit type $G / K_{j}$ in $X$ of the groups $\underline{F}\left(G / K_{j}\right)$. This follows by restricting Eq. (2.2) to the full subcategory $\operatorname{Or}(G, \mathfrak{F}(X))$, with $\mathfrak{F}(X)$ the family of subgroups of $G$ which occur as stabilizers of the $G$-action on $X[50]$.

The $\mathbb{Z}$-graded group $C_{G}^{\bullet}(X, \underline{F})=\bigoplus_{n \in \mathbb{Z}} C_{G}^{n}(X, \underline{F})$ inherits a coboundary operator $\delta$, and hence the structure of a cochain complex, from the boundary operator on cellular chains. To a natural transformation $f: \underline{C_{n}}(X) \rightarrow \underline{F}$, one associates the natural transformation $\delta f$ defined by

$$
\begin{aligned}
\delta f(G / H): C_{n}\left(X^{H}\right) & \longrightarrow \underline{F}(G / H) \\
\sigma & \longmapsto f(G / H)(\partial \sigma)
\end{aligned}
$$

for $\sigma \in C_{n-1}\left(X^{H}\right)$, with naturality induced from that of the cellular boundary operator $\partial$. Then the Bredon cohomology of $X$ with coefficient system $\underline{F}$ is defined as

$$
\mathrm{H}_{G}^{\bullet}(X ; \underline{F}):=\mathrm{H}\left(C_{G}^{\bullet}(X, \underline{F}), \delta\right) .
$$

This defines a $G$-cohomology theory. See ref. [44] for the proof that $\mathrm{H}_{G}^{\bullet}(X ; \underline{F})$ is an equivariant cohomology theory, i.e., for the definition of the induction structure. One can also define cohomology groups by restricting the functors in Eq. (2.2) to a subcategory $\operatorname{Or}(G, \mathfrak{F})$. The definition of Bredon cohomology is independent of $\mathfrak{F}$ as long as $\mathfrak{F}$ contains the family $\mathfrak{F}(X)$ of stabilizers [50]. This fact is useful in explicit calculations. In particular, by taking $\mathfrak{F}=H$ to consist of a single subgroup, one shows that the Bredon cohomology of $G$-homogeneous spaces is given by

$$
\mathrm{H}_{G}^{\bullet}(G / H ; \underline{F})==\mathrm{H}_{G}^{0}(G / H ; \underline{F})==\underline{F}(G / H) .
$$

Example 2.1 (Trivial group). When $G=e$ is the trivial group, i.e., in the non-equivariant case, the functors $\underline{C}_{n}(X)$ and $\underline{F}$ can be identified with the abelian groups $C_{n}(X)=$ $\underline{C}_{n}(X)(e)$ and $F=\underline{F}(e)$. Then

$$
C_{e}^{n}(X, F)=C^{n}(X, F)
$$


and one has $\mathrm{H}_{e}^{n}(X ; \underline{F})=\mathrm{H}\left(C^{n}(X, F), \delta\right)$, i.e., the ordinary $n^{\text {th }}$ cohomology group of $X$ with coefficients in $F$.

Example 2.2 (Free action). If the $G$-action on $X$ is free, then all stabilizers $K_{j}$ are trivial and $X^{H}=\emptyset$ for every $H \leq G, H \neq e$. In this case one may take $\mathfrak{F}=e$ to compute the cochain complex

$$
C_{G}^{\bullet}(X, \underline{F}) \cong \operatorname{Hom}_{G}\left(C_{\bullet}(X), \underline{F}(G / e)\right)
$$

and so the Bredon cohomology $\mathrm{H}_{G}^{\bullet}(X ; \underline{F})$ coincides with the equivariant cohomology

$$
\mathrm{H}_{G}^{\bullet}(X ; \underline{F}(G / e))
$$

of $X$ with coefficients in the $G$-module $\underline{F}(G / e)=\underline{F}(G)$. In the case of the constant functor $\underline{F}=\underline{\mathbb{Z}}$, with $\underline{\mathbb{Z}}(G / H)=\mathbb{Z}$ for every $H \leq \bar{G}$ and the value on morphisms in $\operatorname{Or}(G)^{\text {op }}$ given by the identity homomorphism of $\mathbb{Z}$, this group reduces to the ordinary cohomology $\mathrm{H}^{\bullet}(X / G ; \mathbb{Z})$.

Example 2.3 (Trivial action). If the $G$-action on $X$ is trivial, then the collection of isotropy groups $K_{j}$ for the $G$-action is the set of all subgroups of $G$ and $X^{H}=X$ for all $H \leq G$. In this case the functor $\underline{C}_{n}(X)$ can be decomposed into a sum over $n$-cells of projective functors $\underline{P}_{K_{j}}$ with $K_{j}=G[50]$, and so one has

$$
\operatorname{Hom} \operatorname{Or}(G)\left(\underline{C}_{n}(X), \underline{F}\right) \cong \operatorname{Hom}(C_{n}(X), \underbrace{}_{\lim } \operatorname{Or}(G)^{\text {op }} \underline{F}(G / H)),
$$

where the inverse limit functor is taken over the opposite category $\operatorname{Or}(G)^{\mathrm{op}}$. It follows that the Bredon cohomology

$$
\mathrm{H}_{G}^{\bullet}(X ; \underline{F})=\mathrm{H}^{\bullet}(X ; \underline{F}(G / G))
$$

is the ordinary cohomology of $X$ with coefficients in the abelian group $\underline{F}(G / G)=$ $\underline{F}(\mathrm{pt})$.

2.3. Representation ring functors. In what follows we will specialize the coefficient system for Bredon cohomology to the representation ring functor $\underline{F}=\underline{R}(-)$ defined on the orbit category $\operatorname{Or}(G)$ by sending the left coset $G / H$ to $R(H)$, the complex representation ring of the group $H$. A morphism (2.1) is sent to the homomorphism $R(K) \rightarrow R(H)$ given by first restricting the representation from $K$ to the subgroup conjugate to $H$, and then conjugating by $a$. Since $\underline{R}(-)$ is a functor to rings, the Bredon cohomology $\mathrm{H}_{G}^{\bullet}(X ; \underline{R}(-))$ naturally has a ring structure. Note that

$$
R(H) \cong \mathrm{K}_{G}^{0}(G / H)=\mathrm{K}_{G}^{\bullet}(G / H),
$$

which follows from the induction structure of Example 1.4 with $X=$ pt and $\alpha$ the subgroup inclusion $H \hookrightarrow G$. By Eq. (2.4) the group (2.5) also coincides with the Bredon cohomology group $\mathrm{H}_{G}^{\bullet}(G / H ; \underline{R}(-))$, which is already an indication that Bredon cohomology is a better relative of equivariant K-theory than Borel cohomology. Indeed, using the induction structure of Example 1.3 one shows that the Borel cohomology

$$
\mathrm{H}_{G}^{\bullet}(G / H)=\mathrm{H}^{\bullet}(B H)
$$


coincides with the cohomology of the classifying space $B H=E H / H$, which computes the group cohomology of $H$ and is typically infinite-dimensional (even for finite groups $H)$. In this paper we will show that the Bredon cohomology $\mathrm{H}_{G}^{\bullet}(X ; \underline{R}(-))$ gives a more precise realization of the stringy orbifold cohomology of $X$ in the context of open string theory.

In the construction of the equivariant Chern character in Sect. 2.4 below, it will be important to represent the rational Bredon cohomology $\mathrm{H}_{G}^{\bullet}(X ; \mathbb{Q} \otimes \underline{R}(-))$ as a certain group of homomorphisms of functors, similarly to the cochain groups (2.2). For this, we introduce another category $\operatorname{Sub}(G)$. The objects of $\operatorname{Sub}(G)$ are the subgroups of $G{ }^{2}$ and the morphisms are given by

$$
\operatorname{Mor}_{S u b}(H, K):=\left\{f: H \rightarrow K \mid \exists g \in G, g H g^{-1} \leq K, f=\operatorname{Ad}_{g}\right\} / \operatorname{Inn}(K)
$$

In particular, there is a functor $\operatorname{Or}(G) \rightarrow \operatorname{Sub}(G)$ which sends the object $G / H$ to $H$ and the morphism (2.1) in $\operatorname{Or}(G)$ to the homomorphism $\left(g \mapsto a^{-1} g a\right)$ in $\operatorname{Sub}(G)$. If $a$ lies in the centralizer

$$
Z_{G}(H):=\left\{g \in G \mid g^{-1} H g=H\right\}
$$

of $H$ in $G$, then the morphism (2.1) is sent to the identity map. Any functor $\underline{F}$ : $\operatorname{Sub}(G)^{\mathrm{op}} \rightarrow$ Ab can be naturally regarded as a functor on $\operatorname{Or}(G)^{\mathrm{op}}$.

Define the quotient functors $\underline{C} \cdot \bullet^{\mathrm{qt}}(X), \underline{\mathrm{H}}^{\mathrm{qt}}(X): \operatorname{Sub}(G)^{\mathrm{op}} \rightarrow$ Ab by

$$
\underline{C} \bullet{ }^{\mathrm{qt}}(X)(H):=C \bullet\left(X^{H} / Z_{G}(H)\right) \quad \text { and } \quad \underline{\mathrm{H}}^{\mathrm{qt}}(X)(H):=\mathrm{H}_{\bullet}\left(X^{H} / Z_{G}(H)\right) .
$$

For any functor $\underline{F}: \operatorname{Sub}(G)^{\mathrm{op}} \rightarrow \mathrm{Ab}$ one has

$$
\operatorname{Hom}\left(C_{\bullet}\left(X^{H} / Z_{G}(H)\right), \underline{F}(H)\right) \cong \operatorname{Hom}_{Z_{G}(H)}\left(C_{\bullet}\left(X^{H}\right), \underline{F}(H)\right) .
$$

By observing that the centralizer (2.6) is precisely the group of automorphisms of $G / H$ in the orbit category $\operatorname{Or}(G)$ sent to the identity map in the subgroup category $\operatorname{Sub}(G)$, we finally have

$$
C_{G}^{\bullet}(X, \underline{F})=\operatorname{Hom}_{\mathrm{Or}(G)}(\underline{C} \bullet(X), \underline{F}) \cong \operatorname{Hom}_{\operatorname{Sub}(G)}\left(\underline{C} \cdot \underline{\mathrm{qt}}^{\mathrm{t}}(X), \underline{F}\right) .
$$

At this point one can apply Eq. (2.7) to the rational representation ring functor $\underline{F}=$ $\mathbb{Q} \otimes \underline{R}(-)$, which by construction can be regarded as an injective functor $\operatorname{Sub}(G)^{\mathrm{o}} \rightarrow$ $\mathrm{Ab}$, to prove the

Lemma 2.4 ([46]). For any finite group $G$ and any $G$-complex $X$, there exists an isomorphism of rings

$$
\Phi_{X}: \mathrm{H}_{G}^{\bullet}(X ; \mathbb{Q} \otimes \underline{R}(-)) \stackrel{\approx}{\rightarrow} \operatorname{Hom}_{\mathrm{Sub}(G)}\left(\underline{\mathrm{H}}^{\mathrm{qt}}(X), \mathbb{Q} \otimes \underline{R}(-)\right)
$$

\footnotetext{
${ }^{2}$ If $G$ is infinite then one should restrict to finite subgroups of $G$.
} 
2.4. Chern character in equivariant K-theory. Before spelling out the definition of the equivariant Chern character, we recall some basic properties of the equivariant K-theory of a $G$-complex $X$. Let $H$ be a subgroup of $G$, and consider the fixed point subspace of $X$ defined by Eq. (2.3). The action of $G$ does not preserve $X^{H}$, but the action of the normalizer $N_{G}(H)$ of $H$ in $G$ does. If we denote with $i: X^{H} \hookrightarrow X$ the inclusion of $X^{H}$ as a subspace of $X$, and with $\alpha: N_{G}(H) \hookrightarrow G$ the inclusion of $N_{G}(H)$ as a subgroup of $G$, then we naturally have the equality

$$
i(n \cdot x)=\alpha(n) \cdot i(x)
$$

for all $n \in N_{G}(H)$ and $x \in X^{H}$. It follows that the induced homomorphism on equivariant K-theory is a map [58]

$$
i^{*}: \mathrm{K}_{G}^{\bullet}(X) \longrightarrow \mathrm{K}_{N_{G}(H)}^{\bullet}\left(X^{H}\right)
$$

which is called a restriction morphism.

We also need a somewhat less known property [46]. Let $N \triangleleft G$ be a finite normal subgroup, and let $\operatorname{Rep}(N)$ be the category of (isomorphism classes of) irreducible complex representations of $N$. Let $X$ be a (proper) $G / N$-complex, and let $G$ act on $X$ via the projection map $G \rightarrow G / N$. Then for any complex $G$-vector bundle $E \rightarrow X$ and any representation $V \in \operatorname{Rep}(N)$, define $\operatorname{Hom}_{N}(V, E)$ as the vector bundle over $X$ with total space

$$
\operatorname{Hom}_{N}(V, E):=\bigcup_{x \in X} \operatorname{Hom}_{N}\left(V, E_{x}\right),
$$

where $N$ acts on the fibres of $E$ because of the action of $G$ via the projection map. Now if $H \leq G$ is a subgroup which commutes with $N,[H, N]=e$, then one can induce an $H$-vector bundle from $\operatorname{Hom}_{N}(V, E)$ by defining $(h \cdot f)(v)=h \cdot f(v), v \in V$ for any $h \in H$ and any $f \in \operatorname{Hom}_{N}(V, E)$ (remembering that $G$ acts on $E$ ). Hence there is a homomorphism of rings

$$
\Psi: \mathrm{K}_{G}^{\bullet}(X) \longrightarrow \mathrm{K}_{H}^{\bullet}(X) \otimes R(N)
$$

defined on $G$-vector bundles by

$$
\Psi([E]):=\sum_{V \in \operatorname{Rep}(N)}\left[\operatorname{Hom}_{N}(V, E)\right] \otimes[V] .
$$

This homomorphism satisfies some naturality properties which are described in detail in ref. [46]. Note that the sum (2.8) is finite, since $N$ is a finite subgroup.

We are now ready to construct the equivariant Chern character as a homomorphism

$$
\operatorname{ch}_{X}: \mathrm{K}_{G}^{0,1}(X) \longrightarrow \mathrm{H}_{G}^{\text {even,odd }}(X ; \mathbb{Q} \otimes \underline{R}(-))
$$

for any finite proper $G$-complex $X$. The strategy used in ref. [46] is to construct $\mathbb{Z}_{2}$-graded homomorphisms

$$
\operatorname{ch}_{X}^{H}: \mathrm{K}_{G}^{\bullet}(X) \longrightarrow \operatorname{Hom}\left(\mathrm{H}_{\bullet}\left(X^{H} / Z_{G}(H)\right), \mathbb{Q} \otimes R(H)\right)
$$


for any finite subgroup $H$, and then glue them together as $H$ varies through the finite subgroups of $G$. To define the homomorphism (2.9), we first compose the ring homomorphisms

$$
\begin{aligned}
& \mathrm{K}_{G}^{\bullet}(X) \stackrel{i^{*}}{\rightarrow} \mathrm{K}_{N_{G}(H)}^{\bullet}\left(X^{H}\right) \stackrel{\Psi}{\rightarrow} \mathrm{K}_{Z_{G}(H)}\left(X^{H}\right) \otimes R(H) \\
& \stackrel{\pi_{2}^{*} \otimes \mathrm{id}}{\longrightarrow} \mathrm{K}_{Z_{G}(H)}^{\bullet}\left(E G \times X^{H}\right) \otimes R(H),
\end{aligned}
$$

where $\pi_{2}: E G \times X^{H} \rightarrow X^{H}$ is the projection onto the second factor. By using the induction structure of Example 1.4, one then has

$$
\begin{aligned}
& \mathrm{K}_{Z_{G}(H)}\left(E G \times X^{H}\right) \otimes R(H) \stackrel{\approx}{\rightarrow} \mathrm{K}^{\bullet}\left(E G \times Z_{G}(H) X^{H}\right) \otimes R(H) \\
& \stackrel{\text { ch } \otimes \text { id }}{\longrightarrow} \mathrm{H}\left(E G \times{ }_{Z_{G}(H)} X^{H} ; \mathbb{Q} \otimes \pi_{-\bullet} \mathrm{K}\right)^{\bullet} \otimes R(H),
\end{aligned}
$$

where ch is the ordinary Chern character. One finally has

$$
\begin{aligned}
\mathrm{H}^{\bullet}\left(E G \times{ }_{Z_{G}(H)} X^{H} ; \mathbb{Q}\right) \otimes R(H) & \stackrel{\approx}{\rightarrow} \mathrm{H}^{\bullet}\left(X^{H} / Z_{G}(H) ; \mathbb{Q}\right) \otimes R(H) \\
& \cong \operatorname{Hom}\left(\mathrm{H}_{\bullet}\left(X^{H} / Z_{G}(H)\right), \mathbb{Q} \otimes R(H)\right),
\end{aligned}
$$

where the first isomorphism follows from the Leray spectral sequence by observing that the fibres of the projection

$$
E G \times{ }_{Z_{G}(H)} X^{H} \longrightarrow X^{H} / Z_{G}(H)
$$

are all classifying spaces of finite groups, having trival reduced cohomology with $\mathbb{Q}$-coefficients and are therefore $\mathbb{Q}$-acyclic.

The equivariant Chern character is now defined as ${ }^{3}$

$$
\operatorname{ch}_{X}=\bigoplus_{H \leq G} \operatorname{ch}_{X}^{H} .
$$

By using the various naturality properties of the homomorphism (2.8) [46], one sees that $\operatorname{ch}_{X}$ takes values in $\operatorname{Hom}_{\operatorname{Sub}(G)}\left(\underline{\mathrm{H}}^{\mathrm{qt}}(X), \mathbb{Q} \otimes \underline{R}(-)\right)$, and by Lemma 2.4 it is thus a $\mathbb{Z}_{2 \text {-graded map }}$

$$
\operatorname{ch}_{X}: \mathrm{K}_{G}^{\bullet}(X) \longrightarrow \operatorname{Hom}_{\mathrm{Sub}(G)}\left(\underline{\mathrm{H}}^{\mathrm{qt}}(X), \mathbb{Q} \otimes \underline{R}(-)\right) \cong \mathrm{H}_{G}^{\bullet}(X ; \mathbb{Q} \otimes \underline{R}(-)) .
$$

This map is well-defined as a ring homomorphism because all maps involved above are homomorphisms of rings. As with the definition of Bredon cohomology, the sum (2.10) may be restricted to any family of subgroups of $G$ containing the set of stabilizers $\mathfrak{F}(X)$.

To conclude, we have to prove that this map becomes an isomorphism upon tensoring over $\mathbb{Q}$. For this, one proves that the morphism $\mathrm{ch}_{X}$ in Eq. (2.10) is an isomorphism on homogeneous spaces $G / H$, with $H$ a finite subgroup of $G$, and then uses induction on the number of orbit types of cells in $X$ along with the Mayer-Vietoris sequences for the

\footnotetext{
${ }^{3}$ If $G$ is infinite then the direct sum in Eq. (2.10) is understood as the inverse limit functor over the dual subgroup category $\operatorname{Sub}(G)^{\mathrm{op}}$.
} 
pushout squares induced by the attaching $G$-maps (1.2). The isomorphism on $G / H$ is a consequence of the isomorphisms (2.4) and (2.5). The details may be found in ref. [46]. Let $\pi_{-} \bullet \mathrm{K}_{G}(-)$ be the functor on $\operatorname{Or}(G)$ defined by $G / H \mapsto \mathrm{K}_{G}^{\bullet}(G / H)$. Then one has the following

Theorem 2.5. For any finite proper $G$-complex $X$, the Chern character $\mathrm{ch}_{X}$ extends to a natural $\mathbb{Z}$-graded isomorphism of rings

$$
\operatorname{ch}_{X} \otimes \mathbb{Q}: \mathrm{K}_{G}^{\bullet}(X) \otimes \mathbb{Q} \stackrel{\approx}{\rightarrow} \mathrm{H}_{G}\left(X ; \mathbb{Q} \otimes \underline{\pi_{-} \cdot \mathrm{K}_{G}}(-)\right)^{\bullet}
$$

\section{D-Branes and Equivariant K-Cycles}

In this section we will make some remarks concerning the topological classification of D-branes and their charges on global orbifolds of Type II superstring theory with vanishing $H$-flux. Let $X$ be a smooth manifold and $G$ a (finite) group acting by diffeomorphisms of $X$. Ramond-Ramond charges on the global orbifold $[X / G]$ are classified by the equivariant $\mathrm{K}$-theory $\mathrm{K}_{G}^{\bullet}(X)$ as defined in Example 1.4 [34,54,62]. Dually, the equivariant $\mathrm{K}$-homology $\mathrm{K}_{\bullet}^{G}(X)$ leads to an elegant description of fractional D-branes pinned at the orbifold singularities in terms of equivariant K-cycles. In the following we will frequently refer to Appendix B for detailed definitions and technical aspects of equivariant K-homology, focusing instead here on some of the more qualitative aspects of D-branes on orbifolds in this language. In the remainder of this paper we will assume for definiteness that $G$ is a finite group.

3.1. Fractional D-branes. As in the non-equivariant case $G=e[56,57,60]$, a very natural description of D-branes in the orbifold space, which captures the inherent geometrical picture of D-brane states involving wrapped cycles in spacetime, is provided by the topological realization of the equivariant K-homology groups $\mathrm{K}_{\bullet}^{G}(X)$. The cycles for this homology theory, called $G$-equivariant K-cycles, live in an additive category $\mathrm{D}^{G}(X)$ whose objects are triples $(W, E, f)$, where $W$ is a $G$-spin ${ }^{c}$ manifold without boundary, $E$ is a $G$-vector bundle over $W$, and

$$
f: W \longrightarrow X
$$

is a $G$-map. The group $\mathrm{K}_{\bullet}^{G}(X)$ is the quotient of this category by the equivalence relation generated by bordism, direct sum, and vector bundle modification, as detailed in Appendix B. Note that $W$ need not be a submanifold of spacetime. However, since $X$ is a manifold, we can restrict the bordism equivalence relation to differential bordism [56] and assume that the map (3.1) is a differentiable $G$-map in equivariant K-cycles $(W, E, f) \in \mathrm{D}^{G}(X)$. In this way the category $\mathrm{D}^{G}(X)$ extends the standard $\mathrm{K}$-theory classification to include branes supported on non-representable cycles in spacetime. This definition of equivariant K-homology thus gives a concrete geometric model for the topological classification of D-branes $(W, E, f)$ in a global orbifold $[X / G]$ which captures the physical constructions of orbifold D-branes as $G$-invariant states of branes on the covering space $X$. In the subsequent sections we will study the pairing of Ramond-Ramond fields with these D-branes. 
Consider a D-brane localized at a generic point in the orbifold $[X / G]$ with the action of the regular representation of $G$ on the fibres of its Chan-Paton gauge bundle, i.e., the natural action of the group algebra $\mathbb{C}[G]$ as bounded linear operators $\ell^{2}(G) \rightarrow \ell^{2}(G)$. It corresponds to a $G$-orbit of such branes on the leaves $X^{g}=\{x \in X \mid g \cdot x=x\}, g \in G$ of the covering space $X$. At a $G$-fixed point, this brane splits up into a set of fractional branes according to the decomposition of the representation of $G$ on the fibres of the Chan-Paton bundle into irreducible $G$-modules. Stable fractional D-branes correspond to bound states of branes wrapping various collapsed cycles at the fixed points. They are thus stuck at the orbifold points and provide the open string analogs of "twisted sectors".

To formulate this physical construction in the language of equivariant K-cycles ( $W, E, f$ ), let $G^{\vee}$ denote the set of conjugacy classes $[g]$ of elements $g \in G$. It can be regarded as a set of representatives for the isomorphism classes $\pi_{0} \operatorname{Rep}(G)$, where $\operatorname{Rep}(G)$ is the additive category of irreducible complex representations of $G$ which coincides with the category of D-brane boundary conditions at the orbifold points. There is a natural subcategory $\mathrm{D}_{\text {frac }}^{G}(X)$ of $\mathrm{D}^{G}(X)$ consisting of triples $(W, E, f)$ for which $W$ is a $G$-fixed space, i.e., for which

$$
W^{g}=W
$$

for all $g \in G$. By $G$-equivariance this implies $f(W)^{g}=f(W)$ for all $g \in G$, and so the image of the brane worldvolume lies in the subspace

$$
f(W) \subset \bigcap_{g \in G} X^{g} .
$$

This is precisely the set of $G$-fixed points of $X$, and so the objects ( $W, E, f)$ of the category $\mathrm{D}_{\text {frac }}^{G}(X)$ are naturally pinned to the orbifold points. We call $\mathrm{D}_{\text {frac }}^{G}(X)$ the category of "maximally fractional D-branes".

In this case, an application of Schur's lemma shows that the Chan-Paton bundle admits an isotopical decomposition and there is a canonical isomorphism of $G$-bundles

$$
E \cong \bigoplus_{[g] \in G^{\vee}} E_{[g]} \otimes \mathbb{1}_{[g]} \quad \text { with } \quad E_{[g]}==\operatorname{Hom}_{G}\left(\mathbb{1}_{[g]}, E\right),
$$

where $E_{[g]}$ is a complex vector bundle with trivial $G$-action and $\mathbb{1}_{[g]}$ is the $G$-bundle $W \times V_{[g]}$ with $\gamma: G \rightarrow \operatorname{End}\left(V_{[g]}\right)$ the irreducible representation corresponding to the conjugacy class $[g] \in G^{\vee}$. This isotopical decomposition defines the action of $G$ on the Chan-Paton factors of the D-brane, and it implies the well-known isomorphism

$$
\mathrm{K}_{G}^{\bullet}(W) \cong R(G) \otimes \mathrm{K}^{\bullet}(W)
$$

for $G$-fixed spaces $W$ [58]. This is a special case of the homomorphism $\Psi$ defined in Eq. (2.8). From the direct sum relation in equivariant K-homology it follows that a D-brane, represented by a K-cycle $(W, E, f)$, splits at an orbifold point into a sum over irreducible fractional branes represented by the K-cycles $\left(W, E_{[g]} \otimes \mathbb{1}_{[g]}, f\right),[g] \in G^{\vee}$.

It is important to realize that the full category $\mathrm{D}^{G}(X)$ contains much more information, and in particular the fractional D-branes will not generically form a spanning set of $\mathrm{K}$-cycles for the group $\mathrm{K}_{\bullet}^{G}(X)$ (except in some specific examples). However, it follows from the bordism relation in equivariant K-homology that any two $G$-equivariant K-cycles $\left(W_{i}, E_{i}, f_{i}\right), i=0,1$ which are bordant along the same $G$-orbit determine the same element in $\mathrm{K}_{\bullet}^{G}(X)$. This is expected since a purely topological classification 
such as equivariant K-homology cannot capture the positional moduli associated with the regular D-branes in $X / G$.

A related way to understand the role of fractional branes is through the connection between geometric K-homology and bordism theory [56]. Let $\operatorname{MSpin}_{\bullet}^{c}(X)$ be the ordinary $\operatorname{spin}^{c}$ bordism group of $X$, which forgets about the $G$-action and consists of $\operatorname{spin}^{c}$ bordism classes of pairs $(W, f)$. Then there is a map

$$
\begin{aligned}
\operatorname{MSpin}_{\bullet}^{c}(X) \otimes_{\operatorname{MSpin}_{\bullet}^{c}(\mathrm{pt})} \operatorname{Rep}(G) & \longrightarrow \mathrm{D}_{\text {frac }}^{G}(X), \\
(W, f) \otimes V & \longrightarrow(W, W \times V, f),
\end{aligned}
$$

which descends to give a homomorphism

$$
\operatorname{MSpin}_{\bullet}^{c}(X) \otimes_{\operatorname{MSpin}_{\bullet}^{c}(\mathrm{pt})} \mathrm{K}_{\bullet}^{G}(\mathrm{pt}) \longrightarrow \mathrm{K}_{\bullet}^{G}(X) .
$$

When $G=e$ this is the isomorphism of $\mathrm{K}_{\bullet}(\mathrm{pt})$-modules induced by the AtiyahBott-Shapiro orientation, and the map (3.5) determines K-cycle generators in terms of $\operatorname{spin}^{c}$ bordism generators [56]. The equivariant extension of the Atiyah-Bott-Shapiro construction is given in ref. [42] in terms of finite-dimensional $\mathbb{Z}_{2}$-graded $G$-Clifford modules. Since any $G$-Clifford module can be built as a direct sum of tensor products of $G$-modules and ordinary Clifford modules (see Appendix B), there is an isomorphism of $R(G)$-modules $\mathrm{K}_{G}^{\bullet}(\mathrm{pt}) \cong R(G) \otimes \mathrm{K}^{\bullet}(\mathrm{pt})$ and so these representation modules contain no new information about the orbifold group. This seems to suggest that, at least in certain cases, spanning sets of equivariant K-cycles can be taken to lie in the subcategory $\mathrm{D}_{\text {frac }}^{G}(X)$.

3.2. Topological charges. The topological charge of a fractional D-brane, in a given closed string twisted sector of the orbifold string theory on a $G$-spin ${ }^{c}$ manifold $X$, can be computed by using the equivariant Dirac operator theory developed in Appendix B. The equivariant index of the $G$-invariant $\operatorname{spin}^{c}$ Dirac operator $D_{E}^{X}$ coupled to a $G$-vector bundle $E \rightarrow X$ takes values in $\mathrm{K}_{G}^{\bullet}(\mathrm{pt}) \cong R(G)$. We can turn this into a homomorphism on $\mathrm{K}_{\bullet}^{G}(X)$ with values in $\mathbb{Z}$ by composing with the projection $R(G) \rightarrow \mathbb{Z}$ defined by taking the multiplicity of a given representation

$$
\gamma: G \longrightarrow \operatorname{End}\left(V_{\gamma}\right)
$$

of $G$ on a finite-dimensional complex vector space $V_{\gamma}$. There is a corresponding class in the KK-theory group

$$
[\gamma] \in \mathrm{KK}_{\bullet}\left(\mathbb{C}[G], \operatorname{End}\left(V_{\gamma}\right)\right)
$$

which is represented by the Kasparov module $\left(V_{\gamma}, \gamma, 0\right)$ associated with the extension of the representation (3.6) to a complex representation of group ring $\mathbb{C}[G]$. By Morita equivalence, the Kasparov product with $[\gamma]$ is the homomorphism on K-theory

$$
\mathrm{K}_{0}(\mathbb{C}[G]) \longrightarrow \mathrm{K}_{0}\left(\operatorname{End}\left(V_{\gamma}\right)\right) \cong \mathrm{K}_{0}(\mathbb{C}) \cong \mathbb{Z}
$$

induced by $\gamma: \mathbb{C}[G] \rightarrow \operatorname{End}\left(V_{\gamma}\right)$. We may then define a homomorphism

$$
\mu_{\gamma}: \mathrm{K}_{0}^{G}(X) \longrightarrow \mathbb{Z}
$$


of abelian groups by

$$
\mu_{\gamma}([W, E, f])=\operatorname{Index}_{\gamma}\left(f_{*}\left[\not D_{E}^{W}\right]\right):=\operatorname{ass}\left(f_{*}\left[\not D_{E}^{W}\right]\right) \otimes_{\mathbb{C}[G]}[\gamma]
$$

on equivariant K-cycles $(W, E, f) \in \mathrm{D}^{G}(X)$ (and extended linearly), where

$$
\text { ass }: \mathrm{K}_{\bullet}^{G}(X) \longrightarrow \mathrm{K}_{\bullet}(\mathbb{C}[G])
$$

is the analytic assembly map constructed in Appendix B.

3.3. Linear orbifolds. Let us now consider a simple class of examples wherein everything can be made very explicit. Let $V$ be a complex vector space of dimension $\operatorname{dim}_{\mathbb{C}}(V)=d \geq 1$, and let $G$ be a finite subgroup of $\operatorname{SL}(V)$. Our spacetime $X$ is the $G$-space identified with the product

$$
X=\mathbb{R}^{p, 1} \times V,
$$

where $G$ acts trivially on the Minkowski space $\mathbb{R}^{p, 1}$. Fractional D-branes carrying themselves a complex linear representation of $G$, which is a submodule of $\mathbb{R}^{p, 1} \times V$, have worldvolumes $W$ linearly embedded in the subspace $\mathbb{R}^{p, 1}$ and have transverse space given by the orthogonal complement $f(W)^{\perp} \cong V$ with respect to a chosen inner product. Since the space of hermitian metrics is contractible, all topological quantities below are independent of this choice.

As a $G$-space, $V$ is equivariantly contractible to a point and hence its compactly supported equivariant K-theory is given by [4]

$$
\mathrm{K}_{G, \mathrm{cpt}}^{\bullet}(V) \cong \mathrm{K}_{G}^{\bullet}(\mathrm{pt}) \cong R(G)=\mathbb{Z}^{\left|G^{\vee}\right|} .
$$

This group coincides with the Bredon cohomology $\mathrm{H}_{G, \text { cpt }}^{\bullet}(V ; \underline{R}(-))$, owing to the fact that the equivariant Chern character $\mathrm{ch}_{G / H}$ of Sect. 2.4 is the identity map (since the non-equivariant Chern character $\mathrm{ch}=c_{0}: \mathrm{K}^{0}(\mathrm{pt}) \rightarrow \mathrm{H}^{0}(\mathrm{pt} ; \mathbb{Z})$ is the identity map). It follows that the fractional D-branes, as defined by elements of equivariant K-theory, can be identified with representations of the orbifold group ${ }^{4}$

$$
\gamma=\bigoplus_{a=1}^{\left|G^{\vee}\right|} N_{a} \gamma_{a}
$$

consisting of $N_{a} \geq 0$ copies of the $a^{\text {th }}$ irreducible representation

$$
\gamma_{a}: G \longrightarrow \operatorname{End}\left(V_{a}\right), \quad a==1, \ldots,\left|G^{\vee}\right|,
$$

which defines the action of $G$ on the fibres of the Chan-Paton bundle. More precisely, each irreducible fractional brane is associated to the $G$-bundle $V \times V_{a}$ over $V$.

By Poincaré duality, it follows from Proposition 2.1 of ref. [56] that a basis for the equivariant K-homology group $\mathrm{K}_{\bullet}^{G}(V)$ is provided by the geometric equivariant K-cycles $\left(V, V \times V_{a}, \mathrm{id}_{V}\right), a=1, \ldots,\left|G^{\vee}\right|$. By $G$-homotopy invariance [56, Lemma 1.4]

\footnotetext{
4 If the transverse space $V$ is instead a real linear $G$-module, then throughout one should restrict to the subring of $R(G)$ consisting of representations associated to conjugacy classes $[g] \in G^{\vee}$ for which the centralizer $Z_{G}(g)$ acts on the fixed point subspace $V^{g}$ by oriented automorphisms [40]. This will follow immediately from the isomorphism (4.7) below with $X=V$.
} 
these cycles can be contracted to [pt, $\left.V_{a}, i\right]$, where $i$ is the inclusion of a point pt $\subset V$ whose induced homomorphism

$$
i_{*}: \mathrm{K}_{\bullet}^{G}(\mathrm{pt}) \longrightarrow \mathrm{K}_{\bullet}^{G}(V)
$$

can be taken to be the identity map $R(G) \rightarrow R(G)$. This is simply the physical statement that the stable fractional branes in this case are D0-branes in Type IIA string theory (the Type IIB theory containing no such states). The $G$-invariant Dirac operator $\not_{V_{a}}^{\mathrm{pt}}$ is just Clifford multiplication twisted by the $G$-module $V_{a}$, and thus the topological charges (3.7) of the corresponding fractional branes in the twisted sector labelled by $b$ are given by

$$
\mu_{b}\left(\left[\mathrm{pt}, V_{a}, i\right]\right)==\operatorname{Index}_{\gamma_{b}}\left(\left[\not D_{V_{a}}^{\mathrm{pt}}\right]\right)==\left[V_{a} \otimes\left(\Delta^{+}-\Delta^{-}\right)\right] \otimes_{\mathbb{C}[G]}\left[\gamma_{b}\right],
$$

where $\Delta^{ \pm}$are the half-spin representations of $\mathrm{SO}(2 d)$ on $V \cong \mathbb{C}^{d}$ regarded as $\mathbb{C}[G]$ modules. Acting on the character ring the projection gives $[W] \otimes_{\mathbb{C}[G]}\left[\gamma_{b}\right]=\chi_{W}\left(g_{b}\right)$, where $\chi_{W}: G \rightarrow \mathbb{C}$ is the character of the $G$-module $W$ and $\left[g_{b}\right] \in G^{\vee}$ is the conjugacy class corresponding to the irreducible representation $\gamma_{b}$.

\section{Delocalization and Ramond-Ramond Couplings}

The purpose of this section is to describe the delocalization of Bredon cohomology and the equivariant Chern character, introduced in Sect. 2, and to apply it to the study of the coupling between Ramond-Ramond potentials and the D-branes of the previous section. In the following we will require some standard facts concerning string theory on global orbifolds, particularly its low-energy field theory content. For more details see refs. [24,25].

4.1. Closed string spectrum. The boundary states corresponding to the fractional branes constructed in Sect. 3 have components in the twisted sectors of the closed string Hilbert space $\mathcal{H}$ of orbifold string theory on $X$. The closed string is an embedding $x$ : $\mathbb{S}^{1} \times \mathbb{R} \rightarrow X$ of the worldsheet cylinder, with local coordinates $(\sigma, \tau) \in \mathbb{S}^{1} \times \mathbb{R}$, into the $G$-spin ${ }^{c}$ spacetime manifold $X$. The Hilbert space $\mathcal{H}$ of physical string states decomposes into a direct sum over twisted sectors, each characterized by a conjugacy class, as

$$
\mathcal{H}=\bigoplus_{[g] \in G^{\vee}} \mathcal{H}_{[g]}
$$

with only $G$-invariant states surviving in each superselection sector $\mathcal{H}_{[g]}$. Actually, the Hilbert space factorizes into one sector for each element of the group $G$, but the action of $G$ mixes the sectors within a given conjugacy class. The subspaces in Eq. (4.1) are thus defined as

$$
\mathcal{H}_{[g]}:=\bigoplus_{h \in[g]} \mathcal{H}_{h},
$$

where $\mathcal{H}_{h}$ is the subspace of states induced by the twisted string field boundary condition

$$
x(\sigma+2 \pi, \tau)=h \cdot x(\sigma, \tau)
$$


with an analogous condition on the worldsheet fermion fields (using a lift $\hat{G}$ of the orbifold group). Then $G$ acts on the subspace $\mathcal{H}_{[g]}$, and projecting onto $G$-invariant states in $\mathcal{H}_{[g]}$ is equivalent to projecting onto $Z_{G}(h)$-invariant states in $\mathcal{H}_{h}$ for any $h$ in $[g]$.

The low-energy limit of Type II orbifold superstring theory on $X$ contains RamondRamond fields $C_{[g]}$ coming from the various twisted sectors. The twisted boundary conditions (4.2) on the string embedding map imposes constraints on the low-energy spectrum. For example, the untwisted sector given by $g=e$ contains Ramond-Ramond fields defined on the entire spacetime manifold $X$, while the twisted sector represented by $g \neq e$ gives rise to fields defined only on the fixed point submanifold $X^{g}$. The GSO projection then enforces the properties that the Ramond-Ramond form potentials $C_{[g]}$ determine self-dual fields in each twisted sector, and that they be of odd degree in Type IIA theory and of even degree in Type IIB theory.

The Ramond-Ramond fields can thus be "organised" into the differential complex

$$
\Omega_{G}^{\bullet}(X ; \mathbb{R}):=\bigoplus_{[g] \in G^{\vee}} \Omega^{\bullet}\left(X^{g} ; \mathbb{R}\right)^{Z_{G}(g)} .
$$

Here we consider only fields coming from inequivalent twisted sectors and make a choice of submanifold $X^{g}$, since for any conjugate element $h \in[g]$ there is a diffeomorphism $X^{g} \cong X^{h}$. (No choice is needed in the case in which $G$ is an abelian group.) As $\mathrm{d} \circ g^{*}=g^{*} \circ \mathrm{d}$ for all $g \in G$, the derivation is given by

$$
\mathrm{d}_{G}:=\bigoplus_{[g] \in G^{\vee}} \mathrm{d}_{g},
$$

where $\mathrm{d}_{g}=\mathrm{d}: \Omega^{\bullet}\left(X^{g} ; \mathbb{R}\right) \rightarrow \Omega^{\bullet}\left(X^{g} ; \mathbb{R}\right)$ is the usual de Rham exterior derivative. Note that only the centralizer subgroup of $g$ in $G$ acts (properly) on $X^{g}$.

4.2. Delocalization of Bredon cohomology. We will now show how Bredon cohomology can be used to compute the cohomology of the complex (4.3) of orbifold RamondRamond fields by giving a delocalized description of Bredon cohomology with real coefficients, following refs. [50 and 46] where further details can be found. This is the stringy orbifold cohomology of $X$, defined as the ordinary (real) cohomology of the orbifold resolution $\widetilde{X}=\bigsqcup_{[g] \in G^{\vee}} X^{g} / Z_{G}(g)$. Note that there is a natural surjective map $\pi: \widetilde{X} \rightarrow X$ defined by $(x,[g]) \mapsto x$, and a natural injection $X \hookrightarrow \widetilde{X}$ into the connected component of $\widetilde{X}$ corresponding to the untwisted sector $[g]=[e]$.

Denote with $\underline{\mathbb{R}}(-)$ the real representation ring functor $\mathbb{R} \otimes \underline{R}(-)$ on the orbit category $\operatorname{Or}(G)$. Let $\langle G\rangle$ denote the set of conjugacy classes $[C]$ of cyclic subgroups $C$ of $G$. Let $\underline{\mathbb{R}}_{C}(-)$ be the contravariant functor on $\operatorname{Or}(G)$ defined by $\underline{\mathbb{R}}_{C}(G / H)=0$ if $[C]$ contains no representative $g C g^{-1}<H$, and otherwise $\mathbb{R}_{C}(G / \bar{H})$ is isomorphic to the cyclotomic field $\mathbb{R}\left(\zeta_{|C|}\right)$ over $\mathbb{R}$ generated by the primitive root of unity $\zeta_{|C|}$ of order $|C|$. A standard result from the representation theory of finite groups then gives a natural splitting

$$
\underline{\mathbb{R}}(-)=\bigoplus_{[C] \in\langle G\rangle} \underline{\mathbb{R}}_{C}(-) .
$$

By definition, for any module $\underline{M}(-)$ over the orbit category one has

$$
\begin{aligned}
\operatorname{Hom}_{\operatorname{Or}(G)}\left(\underline{M}(-), \underline{\mathbb{R}}_{C}(-)\right) & \cong \operatorname{Hom}_{N_{G}(C)}\left(\underline{M}(G / C), \underline{\mathbb{R}}_{C}(G / C)\right) \\
& \cong \underline{M}(G / C) \otimes_{N_{G}}(C) \underline{\mathbb{R}} C(G / C),
\end{aligned}
$$


where the normalizer subgroup $N_{G}(C)$ acts on $\underline{\mathbb{R}}_{C}(G / C) \cong \mathbb{R}\left(\zeta_{|C|}\right)$ via identification of a generator of $C$ with $\zeta_{|C|}$.

These facts together imply that the cochain groups (2.2) with $\underline{F}=\underline{\mathbb{R}}(-)$ admit a splitting given by

$$
C_{G}^{\bullet}(X, \underline{\mathbb{R}}(-)) \cong \bigoplus_{[C] \in\langle G\rangle} C^{\bullet}\left(X^{C}\right) \otimes_{N_{G}(C)} \underline{\mathbb{R}}_{C}(G / C) .
$$

As the centralizer $Z_{G}(C)$ acts properly on $X^{C}$, the natural map

$$
\begin{aligned}
& \bigoplus_{[C] \in\langle G\rangle} C^{\bullet}\left(X^{C}\right) \otimes_{N_{G}(C)} \underline{\mathbb{R}}_{C}(G / C) \longrightarrow \bigoplus_{[C] \in\langle G\rangle} C^{\bullet}\left(X^{C} / Z_{G}(C)\right) \\
& \otimes_{W_{G}(C)} \underline{\mathbb{R}}_{C}(G / C)
\end{aligned}
$$

is a cohomology isomorphism, where $W_{G}(C):=N_{G}(C) / Z_{G}(C)$ is the Weyl group of $C<G$ which acts by translation on $X^{C} / Z_{G}(C)$. Since $\mathbb{R}_{C}(G / C)$ is a projective $\mathbb{R}\left[W_{G}(C)\right]$-module, it follows that for any proper $G$-complex $\bar{X}$ the Bredon cohomology of $X$ with coefficient system $\mathbb{R} \otimes \underline{R}(-)$ has a splitting

$$
\mathrm{H}_{G}^{\bullet}(X ; \mathbb{R} \otimes \underline{R}(-)) \cong \bigoplus_{[C] \in\langle G\rangle} \mathrm{H}^{\bullet}\left(X^{C} / Z_{G}(C) ; \mathbb{R}\right) \otimes_{W_{G}(C)} \underline{\mathbb{R}}_{C}(G / C) .
$$

At this point, we note that the dimension of the $\mathbb{R}$-vector space

$$
\underline{\mathbb{R}}_{C}(G / C)^{W_{G}(C)} \cong \mathbb{R} \otimes_{W_{G}(C)} \underline{\mathbb{R}}_{C}(G / C)
$$

is equal to the number of $G$-conjugacy classes of generators for $C$. We also use the fact that for a finite group $G$ a sum over conjugacy classes of cyclic subgroups is equivalent to a sum over conjugacy classes of elements in $G$, and that $X^{\langle g\rangle}=X^{g}$ and $Z_{G}(\langle g\rangle)=Z_{G}(g)$. One finally obtains a splitting of real Bredon cohomology groups ${ }^{5}$

$$
\mathrm{H}_{G}^{\bullet}(X ; \mathbb{R} \otimes \underline{R}(-)) \cong \bigoplus_{[g] \in G^{\vee}} \mathrm{H}^{\bullet}\left(X^{g} ; \mathbb{R}\right)^{Z_{G}(g)}
$$

into ordinary cohomology groups

$$
\mathrm{H}^{\bullet}\left(X^{g} ; \mathbb{R}\right)^{Z_{G}(g)} \cong \mathrm{H}^{\bullet}\left(X^{g} / Z_{G}(g) ; \mathbb{R}\right) \cong \mathrm{H}\left(\Omega^{\bullet}\left(X^{g} ; \mathbb{R}\right)^{Z_{G}(g)}, \mathrm{d}\right)
$$

with constant coefficients $\mathbb{R}$. The group on the right-hand side of Eq. (4.5) corresponds to the (real) "delocalized equivariant cohomology" $\mathrm{H}^{\bullet}\left(\coprod_{g \in G} X^{g}\right)^{G} \otimes \mathbb{R}$ defined by Baum and Connes [6,9]. Note that this group is (non-canonically) isomorphic to $R(G) \otimes$ $\mathrm{H}^{\bullet}(X ; \mathbb{R})$ when the $G$-action on $X$ is trivial. Furthermore, by using Theorem 2.5 one also has a decomposition for equivariant K-theory with real coefficients given by

$$
\mathrm{K}_{G}^{\bullet}(X) \otimes \mathbb{R} \cong \bigoplus_{[g] \in G^{\vee}}\left(\mathrm{K}^{\bullet}\left(X^{g}\right) \otimes \mathbb{R}\right)^{Z_{G}(g)}
$$

However, this decomposition captures only the torsion-free part of the group $\mathrm{K}_{G}^{\bullet}(X)$.

\footnotetext{
5 This splitting in fact holds over $\mathbb{Q}[50]$.
} 
4.3. Delocalization of the equivariant Chern character. The complex (4.3) of orbifold Ramond-Ramond fields can also be used to provide an explicit geometric description of the (complex) equivariant Chern character defined in Sect. 2.4. We will now explain this construction, referring the reader to ref. [17] for the technical details. Consider a complex $G$-bundle $E$ over $X$ equipped with a $G$-invariant hermitian metric and a $G$-invariant metric connection $\nabla^{E}$. One can then define a closed $G$-invariant differential form

$$
\operatorname{ch}(E) \in \Omega^{\bullet}(X ; \mathbb{C})^{G}
$$

in the usual way by the Chern-Weil construction

$$
\operatorname{ch}(E):=\operatorname{Tr}\left(\exp \left(-F^{E} / 2 \pi \mathrm{i}\right)\right)
$$

where $F^{E}$ is the curvature of the connection $\nabla^{E}$. It represents a cohomology class

$$
[\operatorname{ch}(E)] \in \mathrm{H}^{\bullet}(X ; \mathbb{C})^{G}
$$

in the fixed point subring of the action of $G$ as automorphisms of $\mathrm{H}^{\bullet}(X ; \mathbb{C})$. By using the definition of the homomorphisms (2.9), with $\mathbb{Q}$ substituted by $\mathbb{C}$ and $H=e$, one can establish the equality

$$
[\operatorname{ch}(E)]=\operatorname{ch}_{X}^{e}([E]) .
$$

Let $C<G$ be a cyclic subgroup, and define the cohomology class

$$
[\operatorname{ch}(g, E)] \in \mathrm{H}^{\bullet}\left(X^{C} ; \mathbb{C}\right)^{Z_{G}(C)} \cong \mathrm{H}^{\bullet}\left(X^{C} / Z_{G}(C) ; \mathbb{C}\right) \cong \mathrm{H}\left(\Omega^{\bullet}\left(X^{C} ; \mathbb{C}\right)^{Z_{G}(C)}, \mathrm{d}\right)
$$

represented by

$$
\operatorname{ch}(g, E):=\operatorname{Tr}\left(\gamma(g) \exp \left(-F_{C}^{E} / 2 \pi \mathrm{i}\right)\right)
$$

where $g$ is a generator of $C, F_{C}^{E}$ is the restriction of the invariant curvature two-form $F^{E}$ to the fixed point subspace $X^{C}$, and $\gamma$ is a representation of $C$ on the fibres of the restriction bundle $\left.E\right|_{X^{C}}$ which is an $N_{G}(C)$-bundle over $X^{C}$. The character $\chi_{C}$ naturally identifies $R(C) \otimes \mathbb{C}$ with the $\mathbb{C}$-vector space of class functions $C \rightarrow \mathbb{C}$. By using the splitting (4.4) for complex Bredon cohomology, one can then show that

$$
\operatorname{ch}_{X}^{C}([E])(g)=[\operatorname{ch}(g, E)]
$$

up to the restriction homomorphism $R(C) \otimes \mathbb{C} \rightarrow \mathbb{C}_{C}(G / C)$ of rings with kernel the ideal of elements whose characters vanish on all generators of $C$.

Using Eq. (2.10) we can then define the map

$$
\operatorname{ch}^{\mathbb{C}}: \operatorname{Vect}_{G}^{\mathbb{C}}(X) \longrightarrow \bigoplus_{[g] \in G^{\vee}} \Omega^{\text {even }}\left(X^{g} ; \mathbb{C}\right)^{Z_{G}(g)}
$$

from complex $G$-bundles $E \rightarrow X$ given by

$$
\operatorname{ch}^{\mathbb{C}}(E)=\bigoplus_{[g] \in G^{\vee}} \operatorname{Tr}\left(\gamma(g) \exp \left(-F_{g}^{E} / 2 \pi \mathrm{i}\right)\right) .
$$


At the level of equivariant K-theory, from Theorem 2.5 it follows that this map induces an isomorphism

$$
\operatorname{ch}^{\mathbb{C}}: \mathrm{K}_{G}^{\bullet}(X) \otimes \mathbb{C} \stackrel{\approx}{\rightarrow} \mathrm{H}_{G}\left(X ; \mathbb{C} \otimes \underline{\pi_{-} \bullet \mathrm{K}_{G}}(-)\right)^{\bullet},
$$

where we have used the splitting (4.5). The map (4.6) coincides with the equivariant Chern character defined in ref. [5].

4.4. Wess-Zumino pairings. We now have all the necessary ingredients to define a coupling of the Ramond-Ramond fields to a D-brane in the orbifold $[X / G]$. In this section we will only consider Ramond-Ramond fields which are topologically trivial, i.e., elements of the differential complex (4.3), and use the delocalized cohomology theory above by working throughout with complex coefficients. Under these conditions we can straightforwardly make contact with existing examples in the physics literature and write down their appropriate generalizations.

To this aim, we introduce the bilinear product

$$
\wedge_{G}: \Omega_{G}^{\bullet}(X ; \mathbb{R}) \otimes \Omega_{G}^{\bullet}(X ; \mathbb{R}) \longrightarrow \Omega_{G}^{\bullet}(X ; \mathbb{R})
$$

defined on $\omega=\bigoplus_{[g] \in G^{\vee}} \omega_{[g]}$ and $\eta=\bigoplus_{[g] \in G^{\vee}} \eta_{[g]}$ by

$$
\omega \wedge_{G} \eta:=\bigoplus_{[g] \in G^{\vee}} \omega_{[g]} \wedge_{g} \eta_{[g]},
$$

where $\wedge_{g}=\wedge$ is the usual exterior product on $\Omega^{\bullet}\left(X^{g} ; \mathbb{R}\right)$. There is also an integration

$$
\int_{X}^{G}: \Omega_{G}^{\bullet}(X ; \mathbb{R}) \longrightarrow \mathbb{R}
$$

If $\omega \in \Omega_{G}^{\bullet}(X ; \mathbb{R})$ then we set

$$
\int_{X}^{G} \omega:=\frac{1}{\left|G^{\vee}\right|} \sum_{[g] \in G^{\vee}} \int_{X^{g} / Z_{G}(g)} \omega_{[g]} .
$$

The normalization ensures that $\int_{X}^{G} \omega=\int_{X} \omega$ when $G$ acts trivially on $X$ and $\omega \in \Omega^{\bullet}(X ; \mathbb{R})$ is "diagonal" in $R(G) \otimes \Omega^{\bullet}(X ; \mathbb{R})$.

Suppose now that $f: W \rightarrow X$ is the smooth immersed worldvolume cycle of a wrapped D-brane state $(W, E, f) \in \mathrm{D}^{G}(X)$ in the orbifold $[X / G]$, i.e., $W$ is a $G$ $\operatorname{spin}^{c}$ manifold equipped with a $G$-bundle $E \rightarrow W$ and an invariant connection $\nabla^{E}$ on $E$. We define the Wess-Zumino pairing

$$
\mathrm{WZ}: \mathrm{D}^{G}(X) \times \Omega_{G}^{\bullet}(X ; \mathbb{C}) \longrightarrow \mathbb{C}
$$

between such D-branes and Ramond-Ramond fields as

$$
\mathrm{WZ}((W, E, f), C)=\int_{W}^{G} \tilde{C} \wedge_{G} \operatorname{ch}^{\mathbb{C}}(E) \wedge_{G} \mathcal{R}(W, f),
$$


where $\tilde{C}=f^{*} C$ is the pullback along $f: W \rightarrow X$ of the total Ramond-Ramond field

$$
C=\bigoplus_{[g] \in G^{\vee}} C_{[g]}
$$

and the equivariant Chern character is given by Eq. (4.6) with $\gamma$ giving the action of $G$ on the Chan-Paton factors of the D-brane. The closed worldvolume form $\mathcal{R}(W, f) \in$ $\Omega_{G, \mathrm{cl}}^{\text {even }}(W ; \mathbb{C})$ represents a complex Bredon cohomology class which accounts for gravitational corrections due to curvature in the spacetime $X$ and depends only on the bordism class of $(W, f)$. It will be constructed explicitly in Sect. 4.7 below in terms of the geometry of the immersed cycle $f: W \rightarrow X$ and of the $G$-bundle $v \rightarrow W$ given by

$$
v==v(W ; f)==f^{*}\left(T_{X}\right) \oplus T_{W} .
$$

It is easily seen that, modulo the curvature contribution $\mathcal{R}(W, f)$, the very natural expression (4.9) reduces to the usual Wess-Zumino coupling of topologically trivial Ramond-Ramond fields to D-branes in the case $G=e$. But even if a group $G \neq e$ acts trivially on the brane worldvolume $W$ (or on the spacetime $X$ ), there can still be additional contributions to the usual Ramond-Ramond coupling if $E$ is a non-trivial $G$-bundle. This is the situation, for instance, for fractional D-branes

$$
(W, E, f) \in \mathrm{D}_{\text {frac }}^{G}(X)
$$

placed at orbifold singularities. In this case, we may use the isotopical decomposition (3.3) of the Chan-Paton bundle along with Eq. (3.2). Then the Wess-Zumino pairing (4.9) descends to a pairing

$$
\mathrm{WZ}_{\text {frac }}: \mathrm{D}_{\text {frac }}^{G}(X) \times \Omega_{G}^{\bullet}(X ; \mathbb{C}) \longrightarrow \mathbb{C}
$$

with the additive subcategory of fractional branes at orbifold singularities given by

$\mathrm{WZ}_{\mathrm{frac}}((W, E, f), C)=\int_{W}\left(\frac{1}{\left|G^{\vee}\right|} \sum_{[g] \in G^{\vee}} \tilde{C}_{[g]} \wedge \operatorname{ch}\left(E_{[g]}\right) \chi_{\gamma}(g)\right) \wedge \mathcal{R}(W, f)$,

where $\chi_{\gamma}: G \rightarrow \mathbb{C}$ is the character of the representation $\gamma$ and $\mathcal{R}(W, f) \in \Omega_{\mathrm{cl}}^{\mathrm{even}}(W ; \mathbb{C})$. One can immediately read off from the Wess-Zumino action (4.11) the Ramond-Ramond charges of D0-branes, and the state corresponding to the representation $\gamma$ has (fractional) charge

$$
Q_{\gamma}^{[g]}=\frac{\chi_{\gamma}(g)}{\left|G^{\vee}\right|}
$$

with respect to the twisted Ramond-Ramond one-form field $C_{[g]}^{(1)}$. These charges agree with both those of an open string disk amplitude computation and a boundary state analysis for fractional D0-branes [21]. Our general formula (4.9) includes also the natural extension to the Ramond-Ramond couplings of regular D-branes which move freely in the bulk of $X$ under the action of $G$, as well as to other non-BPS D-brane states such as truncated branes. 
4.5. Linear orbifolds. We will now "test" our definition (4.9) on the class of examples considered in Sect. 3.3. These are flat orbifolds for which there are no non-trivial curvature contributions, i.e., $\mathcal{R}(W, f)=1$. Let us specialize to the case of cyclic orbifolds having twist group $G=\mathbb{Z}_{n}$ with $n \geq d$. In this case, as $\mathbb{Z}_{n}$ is an abelian group, one has $\mathbb{Z}_{n}^{\vee}=\mathbb{Z}_{n}$ (setwise) and we can label the non-trivial twisted sectors of the orbifold string theory on $X$ by $k=1, \ldots, n-1$. The untwisted sector is labelled by $k=0$. We take a generator $g$ of $\mathbb{Z}_{n}$ whose action on $V \cong \mathbb{C}^{d}$ is given by

$$
g \cdot\left(z^{1}, \ldots, z^{d}\right):=\left(\zeta^{a_{1}} z^{1}, \ldots, \zeta^{a_{d}} z^{d}\right)
$$

where $\zeta=\exp (2 \pi \mathrm{i} / n)$ and $a_{1}, \ldots, a_{d}$ are integers satisfying $a_{1}+\cdots+a_{d} \equiv 0 \bmod n{ }^{6}$ In this case the action of any element in $\mathbb{Z}_{n}$ has only one fixed point, an orbifold singularity at the origin $(0, \ldots, 0)$. Hence for any $g \neq e$ one has

$$
X^{g} \cong \mathbb{R}^{p, 1}
$$

and the differential complex (4.3) of orbifold Ramond-Ramond fields is given by

$$
\Omega_{\mathbb{Z}_{n}}^{\bullet}(X ; \mathbb{R})=\Omega^{\bullet}(X ; \mathbb{R}) \oplus\left(\bigoplus_{k=1}^{n-1} \Omega^{\bullet}\left(\mathbb{R}^{p, 1} ; \mathbb{R}\right)\right)
$$

Consider now a D-brane $(W, E, f) \in \mathrm{D}_{\text {frac }}^{\mathbb{Z}_{n}}(X)$ with worldvolume cycle $f(W) \subset$ $\mathbb{R}^{p, 1}$ placed at the orbifold singularity, i.e., $f: W \rightarrow \mathbb{R}^{p, 1} \times(0, \ldots, 0) \subset X$. Let the generator $g$ act on the fibres of the Chan-Paton bundle $E \rightarrow W$ in the $n$-dimensional regular representation $\gamma(g)_{i j}=\zeta^{i} \delta_{i j}$. The action on worldvolume fermion fields is determined by a lift $\hat{\mathbb{Z}}_{n}$ acting on the spinor bundle $S \rightarrow W$. Then the pairing (4.9) contains the following terms. First of all, we have the coupling of the untwisted Ramond-Ramond fields to $W$ given by

$$
\int_{W} \tilde{C} \wedge \operatorname{Tr}\left(\exp \left(-F^{E} / 2 \pi \mathrm{i}\right)\right),
$$

which is just the usual Wess-Zumino coupling and hence the Ramond-Ramond charge of a regular (bulk) brane is 1 as expected. Then there are the contributions from the twisted sectors, which by recalling Eq. (3.2) are given by the expression

$$
\int_{W} \frac{1}{n} \sum_{k=1}^{n-1} \tilde{C}_{k} \wedge \operatorname{Tr}\left(\gamma\left(g^{k}\right) \exp \left(-F^{E} / 2 \pi \mathrm{i}\right)\right),
$$

where $g^{k}$ is an element of $\mathbb{Z}_{n}$ of order $k$. Since $\gamma\left(g^{k}\right)_{i i}=\zeta^{i k}$, the coupling in this case is determined by a discrete Fourier transform of the fields $\tilde{C}_{k}$ over the group $\mathbb{Z}_{n}$. The brane associated with the $i^{\text {th }}$ irreducible representation of $\mathbb{Z}_{n}$ has charge $\zeta^{i k} / n$ with respect to the Ramond-Ramond field in the $k^{\text {th }}$ twisted sector. For $d=2$ and $d=3$ this pairing agrees with and uniformizes the gauge field couplings computed in refs. [26 and 27], respectively.

\footnotetext{
${ }^{6}$ Both the requirement that the representation $V$ be complex and the form of the $G$-action are physical inputs ensuring that the closed string background $X$ preserves a sufficient amount of supersymmetry after orbifolding.
} 
4.6. An equivariant Riemann-Roch formula. Let $X, W$ be smooth compact $G$-manifolds, and $f: W \rightarrow X$ a smooth proper $G$-map. If we want to make sense of the equations of motion for the Ramond-Ramond field $C$, which is a quantity defined on the spacetime $X$, then we need to pushforward classes defined on the brane worldvolume $W$ to classes defined on the spacetime. This will enable the construction of RamondRamond currents in Sect. 6 induced by the background and D-branes which appear as source terms in the Ramond-Ramond field equations. Some technical details of the constructions below are provided in Appendix $\mathrm{C}$.

Consider first the non-equivariant case $G=e$. Let $v \rightarrow W$ be the $\mathbb{Z}_{2}$-graded bundle (4.10), i.e., the KO-theory class of $v$ is the virtual bundle $[v]=f^{*}\left[T_{X}\right]-\left[T_{W}\right] \in$ $\mathrm{KO}^{0}(W)$. We assume that $v$ is even-dimensional and endowed with a $\operatorname{spin}^{c}$ structure (this is automatic if both $X$ and $W$ are $\operatorname{spin}^{c}$ ). Then, as reviewed in Appendix C, one can define the Gysin homomorphism in ordinary K-theory

$$
f_{!}^{\mathrm{K}}: \mathrm{K}^{\bullet}(W) \longrightarrow \mathrm{K}^{\bullet}(X) \text {. }
$$

Using the orientations on $X$ and $W$ one has Poincaré duality in ordinary cohomology, inducing a Gysin homomorphism

$$
f_{!}^{\mathrm{H}}: \mathrm{H}^{\bullet}(W ; \mathbb{Q}) \longrightarrow \mathrm{H}^{\bullet}(X ; \mathbb{Q}),
$$

where here we consider the $\mathbb{Z}_{2}$-grading given by even and odd degree.

The pushforward homomorphisms in K-theory and in cohomology, under the conditions stated above, are related by the Riemann-Roch theorem which states that

$$
\operatorname{ch}\left(f_{!}^{\mathrm{K}}(\xi)\right)=f_{!}^{\mathrm{H}}\left(\operatorname{ch}(\xi) \smile \operatorname{Todd}(v)^{-1}\right)
$$

for any class $\xi$ in $\mathrm{K}^{\bullet}(W)$. Here $\operatorname{Todd}(E) \in \Omega_{\mathrm{cl}}^{\mathrm{even}}(W ; \mathbb{C})$ denotes the Todd genus characteristic class of a hermitian vector bundle $E$ over $W$, whose Chern-Weil representative is

$$
\operatorname{Todd}(E)=\sqrt{\operatorname{det}\left(\frac{F^{E} / 2 \pi \mathrm{i}}{\tanh \left(F^{E} / 2 \pi \mathrm{i}\right)}\right)},
$$

where $F^{E}$ is the curvature of a hermitian connection $\nabla^{E}$ on $E$. The Todd class of the $\mathbb{Z}_{2}$-graded bundle (4.10) can be computed by using multiplicativity, naturality and invertibility to get $\operatorname{Todd}(v)=f^{*} \operatorname{Todd}\left(T_{X}\right) / \operatorname{Todd}\left(T_{W}\right)$. Thus the Chern character does not commute with the Gysin pushforward maps, and the defect in the commutation relation is precisely the Todd genus of the bundle $v$. This "twisting" by the bundle $v$ over the D-brane contributes in a crucial way to the Ramond-Ramond current in the non-equivariant case $[19,49,54]$.

Let us now attempt to find an equivariant version of the Riemann-Roch theorem. As the morphism $f: W \rightarrow X$ is $G$-equivariant, the $\mathbb{Z}_{2}$-graded bundle $v$ is itself a $G$-bundle with even $G$-action. We assume that $v$ is $\mathrm{K}_{G}$-oriented. This requirement is just the Freed-Witten anomaly cancellation condition [30] in this case, generalized to global worldsheet anomalies for D-branes represented by generic $G$-equivariant K-cycles. It enables, analogously to the non-equivariant case, the construction of an equivariant Gysin homomorphism

$$
f_{!}^{\mathrm{K}_{G}}: \mathrm{K}_{G}^{\bullet}(W) \longrightarrow \mathrm{K}_{G}^{\bullet}(X) .
$$


We will demonstrate that, under some very special conditions, one can construct a complex Bredon cohomology class which is analogous to the Todd genus and which plays the role of the equivariant commutativity defect as above. Let us suppose that the $G$-action has the property that for any element $g \in G$, the $N_{G}(g)$-bundle $v^{g}=$ $v(W ; f)^{g} \rightarrow W^{g}$ is the $\mathbb{Z}_{2}$-graded bundle $v^{g}=\left.f^{*}\right|_{W^{g}}\left(T_{X^{g}}\right) \oplus T_{W^{g}}$ over the immersion $\left.f\right|_{W^{g}}: W^{g} \rightarrow X^{g}$ with a $Z_{G}(g)$-invariant $\operatorname{spin}^{c}$ structure. Note that these are highly non-trivial conditions, because for an arbitrary $G$-bundle $E \rightarrow X$ one is not even guaranteed in general that $E^{g} \rightarrow X^{g}$ is a vector bundle, as the dimension of the fibre may jump from point to point. As a simple example of what can happen, ${ }^{7}$ let $X=\mathbb{R}$ and $G=\mathbb{R}^{+}$be the group of positive reals under multiplication. Consider the $G$-bundle $X \times V \rightarrow X$ given by projection onto the first factor, where $V$ is a finite-dimensional real vector space and the $G$-action is

$$
g \cdot(x, v)=\left(x, g^{x} v\right)
$$

for all $g \in G$. For any $g \neq 1,(X \times V)^{g}$ is not a fibre bundle over $X^{g}=X$, as the $G$-invariant fibre space over $x=0$ is $V$ while it is the null vector over any other point.

When $G$ is a finite group, one can apply a construction due to Atiyah and Segal [5]. If $E$ is a complex $G$-vector bundle over $X$, its restriction to the fixed point subspace $X^{g}$ for any $g \in G$ carries a representation of the normalizer subgroup $N_{G}(g)$ fibrewise. We can thus decompose $\left.E\right|_{X^{g}}$ into a Whitney sum of sub-bundles $E_{\alpha}=\operatorname{Hom}_{g}\left(\mathbb{1}_{\alpha},\left.E\right|_{X^{g}}\right) \otimes \mathbb{1}_{\alpha}$ over the eigenvalues $\alpha \in \operatorname{spec}(g) \subset \mathbb{C}$ for the action of $g$ on the fibres of $\left.E\right|_{X^{g}}$, where $\operatorname{Hom}_{g}\left(\mathbb{1}_{\alpha},\left.E\right|_{X^{g}}\right)$ is a $Z_{G}(g)$-bundle over $X^{g}$ and $\mathbb{1}_{\alpha}$ is the $N_{G}(g)$-bundle $X^{g} \times V_{\alpha}$ with $V_{\alpha}$ the corresponding eigenspace. We define the class

$$
\phi_{g}(E)=\sum_{\alpha \in \operatorname{spec}(g)} \alpha\left[E_{\alpha}\right]
$$

in the ordinary K-theory of $X^{g}$ with complex coefficients. By Schur's lemma, every element $h \in Z_{G}(g)$ commuting with $g$ acts as a multiple of the identity on the total space of the bundle $E_{\alpha}$, and so the class obtained in this way is $Z_{G}(g)$-invariant. It follows that the map (4.14) on $\operatorname{Vect}_{G}^{\mathbb{C}}(X)$ induces a homomorphism

$$
\phi_{g}: \mathrm{K}_{G}^{\bullet}(X) \otimes \mathbb{C} \longrightarrow\left(\mathrm{K}^{\bullet}\left(X^{g}\right) \otimes \mathbb{C}\right)^{Z_{G}(g)} .
$$

By setting

$$
\phi=\bigoplus_{[g] \in G^{\vee}} \phi_{g}
$$

we obtain a natural isomorphism leading to the splitting [5]

$$
\mathrm{K}_{G}^{\bullet}(X) \otimes \mathbb{C} \cong \bigoplus_{[g] \in G^{\vee}}\left(\mathrm{K}^{\bullet}\left(X^{g}\right) \otimes \mathbb{C}\right)^{Z_{G}(g)}
$$

The equivariant Chern character (4.7) provides an isomorphism componentwise between the equivariant K-theory group (4.15) and the complex Bredon cohomology of $X$.

${ }^{7}$ We are grateful to J. Figueroa-O'Farrill for suggesting this example to us. 
Suppose now that the equivariant Thom class $\operatorname{Thom}_{G}(v) \in \mathrm{K}_{G \text {,cpt }}^{\bullet}(v)$ can be decomposed according to the splitting (4.15) in such a way that the component in any subgroup

$$
\text { Thom }\left(v^{g}\right) \in\left(\mathrm{K}_{\mathrm{cpt}}^{\bullet}\left(v^{g}\right) \otimes \mathbb{C}\right)^{Z_{G}(g)}
$$

coincides with the (ordinary) Thom class of the $\mathbb{Z}_{2}$-graded bundle $v^{g} \rightarrow W^{g}$. Under these conditions, the equivariant Gysin homomorphism (4.13) constructed in Appendix $\mathrm{C}$ decomposes according to the splitting

$$
f_{!}^{\mathrm{K}_{G}}=\bigoplus_{[g] \in G^{\vee}} f_{g}^{\mathrm{K}}
$$

where $f_{g}^{\mathrm{K}}$ is the $\mathrm{K}$-theoretic Gysin homomorphism associated to the smooth proper map

$$
\left.f\right|_{W^{g}}: W^{g} \longrightarrow X^{g} .
$$

Define the characteristic class $\operatorname{Todd}_{G}$ by

$$
\operatorname{Todd}_{G}(v):=\bigoplus_{[g] \in G^{\vee}} \operatorname{Todd}\left(v^{g}\right),
$$

where Todd is the ordinary Todd genus. This class defines an element of the even degree complex Bredon cohomology of the brane worldvolume $W$. Under the conditions spelled out above, we can now use the equivariant Chern character (4.7) and the usual RiemannRoch theorem for each pair $\left(W^{g}, X^{g}\right)$ to prove the identity

$$
f_{!}^{\mathrm{H}_{G}}\left(\operatorname{ch}^{\mathbb{C}}(\xi) \smile_{G} \operatorname{Todd}_{G}(v)^{-1}\right)=\operatorname{ch}^{\mathbb{C}}\left(f_{!}^{\mathrm{K}_{G}}(\xi)\right)
$$

for any class $\xi \in \mathrm{K}_{G}^{\bullet}(W) \otimes \mathbb{C}$, as all quantities involved in the formula (4.17) are compatible with the $G$-equivariant decompositions given above.

When the geometric conditions assumed above are not met, the equivariant Todd class in the formula (4.17) should be modified by multiplying it with another equivariant characteristic class $\Lambda_{G}(W)$ which reflects non-trivial geometry of the normal bundles $N_{W^{g}}$ to the embeddings $W^{g} \subset W$. This should come from applying a suitable fixed point theorem to the ordinary Riemann-Roch formula (4.12), but we have not found a version which is suitable to our particular equivariant Chern character in the general case on the category of $G$-spaces. When $f$ is the collapsing map $X \rightarrow \mathrm{pt}$, this is the content of the index theorem used in Sect. 4.7 below. The formula (4.17) is, however, directly applicable on the category $\mathrm{D}_{\text {frac }}^{G}(X)$ of fractional D-branes. When $G$ is the cyclic group $\mathbb{Z}_{n}$ as in Sect. 4.5 above, one can apply the Thomasson-Nori fixed point theorem $[53,61]$ to get

$$
\Lambda_{\mathbb{Z}_{n}}(W)=\bigoplus_{k=0}^{n-1} \zeta^{k} \operatorname{ch}\left(\bigwedge_{-1} N_{W^{g^{k}}}^{\vee}\right) \in \mathrm{H}_{\mathbb{Z}_{n}}^{\bullet}(W ; \mathbb{C} \otimes \underline{R}(-))
$$

where

$$
\bigwedge_{-1} N_{W^{g}}=\sum_{l=0}^{\operatorname{codim}\left(W^{g}\right)}(-1)^{l}\left[\bigwedge^{l} N_{W^{g}}\right] \in \mathrm{K}^{0}\left(W^{g}\right) .
$$


4.7. Gravitational pairings. We will now explain how to compute the curvature contributions $\mathcal{R}(W, f) \in \Omega_{G, \mathrm{cl}}^{\mathrm{even}}(W ; \mathbb{C})$ to the Wess-Zumino functional (4.9) for the brane geometries described in Sect. 4.6 above and for vanishing $B$-field. We derive the cancelling form for the Ramond-Ramond gauge anomaly inflow due to chiral fermions on the intersection worldvolume for families of branes using the usual descent procedure [35], which is due to curvature of the spacetime manifold $X$ itself. For this, we must explicitly use the $G$-spin ${ }^{c}$ structure on $X$. The standard mathematical intuition behind this correction is to modify the equivariant Chern character to an isometry with respect to the natural bilinear pairings on equivariant K-theory and Bredon cohomology with complex coefficients $[49,54]$.

The natural sesquilinear pairing between two classes of complementary degrees in complex Bredon cohomology, represented by closed differential forms $\omega, \eta \in \Omega_{G, \mathrm{cl}}^{\bullet}$ $(X ; \mathbb{C})$, is given by $([\omega],[\eta])_{\mathrm{H}_{G}}:=\int_{X}^{G} \bar{\omega} \wedge_{G} \eta$. On the other hand, the natural quadratic form on fractional branes defined by classes in equivariant K-theory, represented by complex $G$-vector bundles $E, F \rightarrow X$, is the topological charge

$$
([E],[F])_{\mathrm{K}_{G}}:=\mu_{1}\left(\left[X, E^{\vee} \otimes F, \mathrm{id}_{X}\right]\right)
$$

of Eq. (3.7) in the untwisted sector corresponding to the representation $\gamma=1: \mathbb{C}[G] \rightarrow$ $\mathbb{C}$ induced from the trivial representation of $G$. This quantity agrees with the natural intersection form on boundary states computed as the $G$-invariant Witten index over open string states suspended between D-branes [51], which counts the difference between the number of positive and negative chirality Ramond ground states and hence computes the required chiral fermion anomaly.

The two bilinear forms are related through the local index theorem which provides a formula for Index $1\left(\left[D_{E}^{X}\right]\right)$ in terms of integrals of characteristic forms over the various singular strata of the orbifold $[X / G]$. It reads [17]

$$
\begin{aligned}
\operatorname{Index}_{1}\left(\left[\not D_{E}^{X}\right]\right)= & \sum_{[g] \in G^{\vee}} \frac{2^{d_{g}}}{|g|} \int_{X^{g} / Z_{G}(g)} \operatorname{ch}(g, E) \wedge \operatorname{Todd}\left(T_{X^{g}}\right) \\
& \wedge \int_{\wedge^{\bullet} N_{X^{g}}} \frac{\operatorname{STr}_{S}(\Delta(g))}{\sqrt{\operatorname{det}(1-N(g)) \operatorname{det}\left(1-N(g) \exp \left(-F^{N_{X}} / 2 \pi \mathrm{i}\right)\right)}},
\end{aligned}
$$

where $d_{g}=\operatorname{dim}(X)-\operatorname{dim}\left(X^{g}\right),|g|$ is the order of the element $g \in G$, and $N(g)$ denotes the action of $g \in G$ on the fibres of the normal bundle $N_{X^{g}}$ to $X^{g}$ in $X$. The determinant is taken over the normal bundle $N_{X^{g}}$ for each $g \in G$, implemented by a BerezinGrassmann integration over the exterior algebra bundle $\bigwedge^{\bullet} N_{X^{g}}$. The form $\Delta(g)$, regarded as an element of $\bigwedge^{\bullet} N_{X^{g}}$ under the symbol map, is the action of $g$ on the fibres of the spinor bundle $\left.S\right|_{X^{g}}$, and $\mathrm{STr}_{S}$ is the supertrace over the endomorphism bundle of $S$.

This formula can be thought of as an equivariant localization of the usual AtiyahSinger index density onto the submanifolds $X^{g} \subset X$ of fixed points of the $G$-action on $X$, with the determinants reflecting the "Euler class" contributions from the non-trivial normal bundles to $X^{g}$. This is the anticipated physical result arising from the closed string twisted Witten index, computed as the partition function on the cylinder with the twisted boundary conditions (4.2). Supersymmetry localizes the computation onto zero modes of the string fields which are constant maps to the submanifolds $X^{h} \subset X$, while modular invariance requires a (weighted) sum over all twisted sectors. The index can be 
rewritten using the equivariant characteristic classes defined as in Eqs. (4.6) and (4.16) to get

$$
\operatorname{Index}_{1}\left(\left[\not D_{E}^{X}\right]\right)=\int_{X}^{G} \operatorname{ch}^{\mathbb{C}}(E) \wedge_{G} \operatorname{Todd}_{G}\left(T_{X}\right) \wedge_{G} \Lambda_{G}(X),
$$

where we have used $\left(T_{X}\right)^{g}=T_{X^{g}}$ and the element of $\Omega_{G, \mathrm{cl}}^{\bullet}(X ; \mathbb{C})$ given by

$$
\begin{aligned}
\Lambda_{G}(X):= & \bigoplus_{[g] \in G^{\vee}} \frac{|g|}{2^{d_{g}}\left|G^{\vee}\right|} \\
& \times \int_{\Lambda^{\bullet} N_{X^{g}} g} \frac{\operatorname{STr}_{S}(\Delta(g))}{\sqrt{\operatorname{det}(1-N(g)) \operatorname{det}\left(1-N(g) \exp \left(-F^{N_{X^{g}}} / 2 \pi \mathrm{i}\right)\right)}}
\end{aligned}
$$

defines a characteristic class in the complex Bredon cohomology of $X$. Note that the integrands of Eq. (4.20) are formally similar to the Chern characters of the virtual bundles (4.18) above.

By using multiplicativity of the equivariant Chern character (4.6) to write

$$
\operatorname{ch}^{\mathbb{C}}\left(E^{\vee} \otimes F\right)=\overline{\operatorname{ch}^{\mathbb{C}}(E)} \wedge_{G} \operatorname{ch}^{\mathbb{C}}(F),
$$

it follows from Eq. (4.19) that the map (4.7) can be turned into an isometry by "twisting" it with the closed differential form $\sqrt{\operatorname{Todd}_{G}\left(T_{X}\right) \wedge_{G} \Lambda_{G}(X)}$, which when pulled back along $f: W \rightarrow X$ gives the required anomaly cancelling form on the brane worldvolume. This should then be combined with the correction $\operatorname{Todd}_{G}(v)^{-1}$ contributed by the $\mathbb{Z}_{2}$-graded bundle (4.10) to the Riemann-Roch formula (4.17). Then under the various conditions spelled out in Sect. 4.6 above, the required map $\mathcal{R}$ in Eq. (4.9) from $G$-spin ${ }^{c}$ bordism classes $[(W, f)]$ to $\Omega_{G, \mathrm{cl}}^{\mathrm{even}}(W ; \mathbb{C})$ is given by

$$
\mathcal{R}(W, f)=\frac{f^{*} \sqrt{\operatorname{Todd}_{G}\left(T_{X}\right) \wedge_{G} \Lambda_{G}(X)}}{\operatorname{Todd}_{G}(\nu)}=\operatorname{Todd}_{G}\left(T_{W}\right) \wedge_{G} f^{*} \sqrt{\frac{\Lambda_{G}(X)}{\operatorname{Todd}_{G}\left(T_{X}\right)}} .
$$

The main new ingredient in this formula is the contribution from the fixed point submanifolds $X^{g} \subset X$, particularly their normal bundle characteristic classes (4.20). This corrects previous topologically trivial, flat space formulas, even for $G$-fixed worldvolumes $W$ (see ref. [34] for example). Note that when the $G$-action on $X$ is trivial, one has $\operatorname{Todd}_{G}\left(T_{X}\right)=\operatorname{Todd}\left(T_{X}\right)$ and $\Lambda_{G}(X)$ is constant.

\section{Orbifold Differential K-Theory}

The main drawback of the delocalized theory of the previous section is that it cannot incorporate the interesting effects of torsion, which have been one of the driving forces behind the K-theory description of D-branes and Ramond-Ramond fluxes, and as such it is desirable to have a description which utilizes the full $R(G)$-module $\mathrm{K}_{G}^{\bullet}(X)$. In this section we will develop an extension of differential K-theory as defined in ref. [37] to incorporate the case of a $G$-manifold. These are the groups needed to extend the analysis of the previous section to topologically non-trivial, real-valued Ramond-Ramond fields. While we do not have a formal proof that this is a proper definition of an equivariant 
differential cohomology theory, we will see that it matches exactly with expectations from string theory on orbifolds and also has the correct limiting properties. For this reason we dub the theory that we define 'orbifold' differential K-theory, deferring the terminology 'equivariant' to a more thorough treatment of our model (we discuss this in more detail in Sect. 5.4 below). In the following we will spell out the definition of differential K-theory groups. The crux of the extensions of these definitions to the equivariant setting will be explicit constructions of the exact sequences they are described by, which are important for physical considerations. We will determine concrete realizations of the various morphisms involved, which are given in a general but abstract framework in ref. [37]. ${ }^{8}$ See refs. [28,32] for an introduction to differential cohomology theories and their applications in physics.

5.1. Differential cohomology theories. Differential K-theory of a manifold is an enrichment of its K-theory, which encodes global topological information, with local geometric information contained in the de Rham complex. Consider a (generalized) cohomology theory $\mathrm{E}^{\bullet}$ defined on the category of smooth manifolds $X$ along with a canonical map

$$
\varphi: \mathrm{E}^{\bullet}(X) \longrightarrow \mathrm{H}\left(X ; \mathbb{R} \otimes \pi_{-\bullet} \mathrm{E}\right)^{\bullet}
$$

which induces an isomorphism

$$
\mathrm{E}^{\bullet}(X) \otimes \mathbb{R} \cong \mathrm{H}\left(X ; \mathbb{R} \otimes \pi_{-\bullet} \mathrm{E}\right)^{\bullet},
$$

i.e., the image of $\varphi$ is a full lattice and its kernel is the torsion subgroup of $\mathrm{E}^{\bullet}(X)$. Then one can define differential E-theory as the cohomology theory $\check{E}^{\bullet}$ which lifts $\mathrm{E}^{\bullet}$ via the pullback square

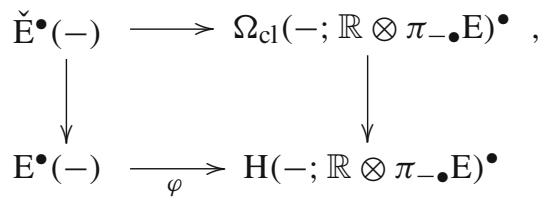

where $\Omega_{\mathrm{cl}}\left(X ; \mathbb{R} \otimes \pi_{-} \bullet \mathrm{E}\right)^{q}$ denotes the real vector space of closed $\mathrm{E}^{\bullet}(\mathrm{pt} ; \mathbb{R})$-valued differential forms $\omega$ on $X$ of total degree $q$, and the right vertical map in the commutative diagram (5.2) is given by sending $\omega$ to its de Rham cohomology class $[\omega]_{\mathrm{dR}}$. A class in $\check{\mathrm{E}}^{q}(X)$ is given by a pair $(\xi, \omega)$, with $\xi \in \mathrm{E}^{q}(X)$ such that

$$
\varphi(\xi)=[\omega]_{\mathrm{dR}},
$$

together with an isomorphism that realizes the equality (5.3) explicitly in $\mathrm{H}(X ; \mathbb{R} \otimes$ $\left.\pi_{-\bullet} \mathrm{E}\right)^{q}$.

In their foundational paper [37] Hopkins and Singer define the differential E-theory associated to any generalized cohomology theory $\mathrm{E}^{\bullet}$, and prove its naturality and homotopy properties. This is done by generalizing the concept of function space in algebraic topology, which can be used to define the cohomology of a space, to that of differential function space, where here the term "differential" typically means something different

\footnotetext{
8 An explicit proof of these exact sequences has been given recently in ref. [18] using the geometric description of differential K-cocyles in terms of bundles with connection. Our proof is more general, but less geometric, as it exploits the realization in terms of maps to classifying spaces.
} 
from differentiable or smooth. Because of this, the differential E-groups are defined in an abstract way and are difficult to realize explicitly. An explicit construction for differential K-theory is given in ref. [37]. In the following we will go through this construction in some detail. This will be our starting point to give a definition of the differential cohomology theory associated to equivariant $\mathrm{K}$-theory $\mathrm{K}_{G}^{\bullet}$, which will reduce to ordinary differential K-theory in the case where the group $G$ is the trivial group. The validity of our definition will be confirmed by explicit construction of the exact sequences, that will also be important in our later physical applications.

5.2. Differential K-theory. Throughout $X$ will denote a smooth manifold. Let Fred be the algebra of Fredholm operators on a separable Hilbert space. Recall that Fred is a classifying space for complex K-theory through the isomorphism

$$
[X, \text { Fred }] \stackrel{\operatorname{Index}(-)}{\longrightarrow} \mathrm{K}^{0}(X)
$$

which associates to any map $f: X \rightarrow$ Fred the index bundle of $f$ in $\mathrm{K}^{0}(X)$. Let

$$
u \in Z^{\text {even }}(\text { Fred; } \mathbb{R})
$$

be a cocycle of even degree which represents the Chern character of the universal bundle. Then for any map $f: X \rightarrow$ Fred representing a complex vector bundle $E \rightarrow X$, the pullback $f^{*} u$ is a representative of $\operatorname{ch}(E)$ in $\mathrm{H}^{\text {even }}(X ; \mathbb{R})$.

The differential K-theory group $\check{\mathrm{K}}^{0}(X)$ is defined to be the set of triples $(c, h, \omega)$, where $c: X \rightarrow$ Fred, $\omega$ is a closed differential form in $\Omega_{\mathrm{cl}}^{\mathrm{even}}(X ; \mathbb{R})$, and $h$ is a cochain in $C^{\text {even-1 }}(X ; \mathbb{R})$ satisfying

$$
\delta h=\omega-c^{*} u .
$$

The cochain $h$ in Eq. (5.4) is precisely the isomorphism refered to in Sect. 5.1 above, which is invisible in the cohomology groups, and in this equation the closed differential form $\omega$ is regarded as a cochain under the de Rham map $\omega \mapsto \int_{(-)} \omega$. Two triples $\left(c_{0}, h_{0}, \omega_{0}\right)$ and $\left(c_{1}, h_{1}, \omega_{1}\right)$ are equivalent if there exists a triple $(c, h, \omega)$ on $X \times[0,1]$, with $\omega=\omega(t)$ constant along $t \in[0,1]$, such that

$$
\left.(c, h, \omega)\right|_{t=0}==\left(c_{0}, h_{0}, \omega_{0}\right) \quad \text { and }\left.\quad(c, h, \omega)\right|_{t=1}==\left(c_{1}, h_{1}, \omega_{1}\right) .
$$

The equivalence (5.5) can be rephrased [28] by requiring that there exists a map

$$
F: X \times[0,1] \longrightarrow \text { Fred }
$$

and a differential form $\sigma \in \Omega^{\text {even-2 }}(X ; \mathbb{R})$ such that

$$
\begin{aligned}
\left.F\right|_{t=0} & =c_{0}, \\
\left.F\right|_{t=1} & =c_{1}, \\
\omega_{1} & =\omega_{0}, \\
h_{1} & =h_{0}+\pi_{*} F^{*} u+\mathrm{d} \sigma,
\end{aligned}
$$

where $\pi: X \times[0,1] \rightarrow X$ is the natural projection. The relations (5.6) say that $c_{0}$ and $c_{1}$ are homotopic maps, hence they represent the same class in $\mathrm{K}^{0}(X)$, and that the 
cochains $h_{0}$ and $h_{1}$ are related by the homotopy that connects the maps $c_{0}$ and $c_{1}$. We also see that the closed form $\omega$ completely characterizes the triple $(c, h, \omega)$.

Borrowing terminology used in representing classes in the differential cohomology $\check{\mathrm{H}}^{2}(X)$ as principal $\mathrm{U}(1)$-bundles with connection, the class $[c] \in \mathrm{K}^{0}(X)$ is called the characteristic class, the closed differential form $\omega$ is called the curvature, while the cochain $h$ is called the holonomy of the triple. From the defining property of the universal cocycle $u$ and Eq. (5.4) it follows that

$$
\operatorname{ch}([c])=[\omega]_{\mathrm{dR}} .
$$

Thus the cohomology class represented by the curvature $\omega$ lies in the image of the (real) Chern character, which is a lattice of maximal rank inside the cohomology group with real coefficients.

Let us now define the differential K-theory group $\check{\mathrm{K}}^{-1}(X)$. Recall that the classifiying space for $\mathrm{K}^{-1}$ is the based loop space $\Omega$ Fred. Thus we need a cocycle

$$
u^{-1} \in Z^{\text {odd }}(\Omega \text { Fred; } \mathbb{R})
$$

which represents the universal odd Chern character. Consider the evaluation map

$$
\text { ev }: \Omega \text { Fred } \times \mathbb{S}^{1} \longrightarrow \text { Fred. }
$$

Then the cocycle $u^{-1}$ is defined by

$$
u^{-1}=\Pi_{*} \mathrm{ev}^{*} u
$$

where $\Pi: \Omega$ Fred $\times \mathbb{S}^{1} \rightarrow \Omega$ Fred is the natural projection. In fact, $u^{-1}$ can be defined as the slant product of $\mathrm{ev}^{*} u$ with the fundamental class of the circle $\mathbb{S}^{1}$, i.e., by integrating the cocycle $\mathrm{ev}^{*} u$ along $\mathbb{S}^{1}$. As above, a class in $\check{\mathrm{K}}^{-1}(X)$ is represented by a triple $(c, h, \omega)$, where $c: X \rightarrow \Omega$ Fred, $\omega$ is a closed differential form in $\Omega_{\mathrm{cl}}^{\text {even-1 }}(X ; \mathbb{R})$, and $h$ is a cocycle in $C^{\text {even-2 }}(X ; \mathbb{R})$ satisfying

$$
\delta h=\omega-c^{*} u^{-1} .
$$

Two triples $\left(c_{0}, h_{0}, \omega_{0}\right)$ and $\left(c_{1}, h_{1}, \omega_{1}\right)$ are equivalent if a relation like that in Eq. (5.5) holds.

In an analogous way one can define the higher differential K-theory groups $\check{\mathrm{K}}^{-n}(X)$ for any positive integer $n$. One can prove that Bott periodicity in complex K-theory induces a periodicity in differential K-theory given by

$$
\check{\mathrm{K}}^{-n}(X) \cong \check{\mathrm{K}}^{-n-2}(X) \text {. }
$$

This periodicity enables one to define the higher differential K-theory groups in positive degrees. The group composition law on $\check{\mathrm{K}}^{-n}(X)$ is given by

$$
\left(c_{1}, h_{1}, \omega_{1}\right)+\left(c_{2}, h_{2}, \omega_{2}\right):=\left(c_{1} \cdot c_{2}, h_{1}+h_{2}, \omega_{1}+\omega_{2}\right),
$$

where the dot denotes pointwise multiplication. The identity element is given by the triple $(\underline{c}, 0,0)$, where throughout $\underline{c}$ denotes any map which is homotopic to the (constant) identity map. To allow for the presence of the characteristic class $\omega$ in the definition, the abelian groups $\check{\mathrm{K}}^{-n}(X)$ are generally infinite-dimensional. The definition of $\check{\mathrm{K}}^{-n}(X)$ 
depends, up to homotopy type and cohomology class, on the choice of classifying space and of universal cocycle $u$ [37].

A key property is the exact sequences which characterize the differential K-theory groups $\check{\mathrm{K}}^{-n}(X)$ for any $n \in \mathbb{Z}$ as extensions of topological $\mathrm{K}$-theory by certain groups of differential forms. In each case the differential K-theory group $\check{\mathrm{K}}^{\bullet}(X)$ is an extension of the setwise fibre product

$$
A_{\mathrm{K}}^{\bullet}(X)=\left\{(\xi, \omega) \in \mathrm{K}^{\bullet}(X) \times \Omega_{\mathrm{cl}}\left(X ; \mathbb{R} \otimes \pi_{-\bullet} \mathrm{K}\right)^{\bullet} \mid \operatorname{ch}(\xi)=[\omega]_{\mathrm{dR}}\right\}
$$

by the torus of topologically trivial flat fields given by

$$
0 \longrightarrow \mathrm{K}^{\bullet-1}(X) \otimes \mathbb{R} / \mathbb{Z} \longrightarrow \check{\mathrm{K}}^{\bullet}(X) \longrightarrow A_{\mathrm{K}}^{\bullet}(X) \longrightarrow 0 .
$$

This will be useful below when we define equivariant differential K-theory.

As in the case of topological K-theory, there are geometrical realizations of the groups $\check{\mathrm{K}}^{-n}(X)$ [28]. In particular, a class in $\check{\mathrm{K}}^{0}(X)$ can be represented by a complex vector bundle $E \rightarrow X$ equipped with a connection $\nabla^{E}$. To the pair $\left(E, \nabla^{E}\right)$ we can associate the triple $(f, \eta, \omega)$, where $f: X \rightarrow B U$ is a map which classifies the bundle $E$, $\omega=\operatorname{ch}\left(\nabla^{E}\right)$ is a Chern-Weil representative of the Chern character of $[E]$, and $\eta$ is a Chern-Simons form such that $\mathrm{d} \eta=f^{*} \omega_{B U}-\omega$ with $\omega_{B U}=\operatorname{ch}\left(\nabla_{B U}\right)$ the Chern character form of the universal bundle $E_{B U} \rightarrow B U$ with respect to the universal connection $\nabla_{B U}$ on $E_{B U}$.

In the following we will define abelian groups that can be thought of as a natural generalization of the differential K-theory of a manifold $X$ acted upon by a (finite) group $G$. In this case one cannot employ the powerful machinery developed in ref. [37], as the equivariant $\mathrm{K}$-theory $\mathrm{K}_{G}^{\bullet}(X)$ is not a cohomology theory defined on the category of manifolds. Instead, we will take as our starting point the explicit definition of the groups $\check{\mathrm{K}}^{-n}(X)$ given above, and naturally generalize it to groups $\check{\mathrm{K}}_{G}^{-n}(X)$ which accommodate the action of the group $G$ in such a way that when $G=e$ is trivial, one has $\check{\mathrm{K}}_{G}^{-n}(X) \cong \check{\mathrm{K}}^{-n}(X)$.

5.3. Orbifold differential forms. We want to generalize the commutative diagram (5.2) to the case in which our underlying cohomology theory $\mathrm{E}^{\bullet}(X)$ is the equivariant $\mathrm{K}$-theory $\mathrm{K}_{G}^{\bullet}(X)$. We first need a homomorphism $\varphi$ from equivariant $\mathrm{K}$-theory to a target cohomology theory which induces an isomorphism when tensored over the reals. For this, we will use the Chern character constructed in Sect. 2.4 with the target cohomology theory given by Bredon cohomology. Then we need a refinement of this cohomology which reduces to the de Rham complex when the group $G$ is trivial. This complex may be thought of as the complex of differential forms on the orbifold $X / G$. For this purpose, we will use the differential complex $\left(\Omega_{G}^{\bullet}(X ; \mathbb{R}), \mathrm{d}_{G}\right)$ defined in Sect. 4.1. Using the delocalization formula (4.5) one shows that this complex is a refinement for Bredon cohomology with real coefficients, in the case when $G$ is a finite group. It comes equipped with a natural product defined in Eq. (4.8). As a refinement for Bredon cohomology, the complex $\Omega_{G}^{\bullet}(X ; \mathbb{R})$ gives a well-defined map

$$
\omega \longmapsto[\omega]_{G-\mathrm{dR}} \in \mathrm{H}\left(\Omega_{G}^{\bullet}(X ; \mathbb{R}), \mathrm{d}_{G}\right) \cong \mathrm{H}_{G}^{\bullet}(X ; \mathbb{R} \otimes \underline{R}(-))
$$

and reduces to the usual de Rham complex of differential forms in the case $G=e$.

There is an alternative complex one could construct which is "manifestly" equivariant, in the sense that its functoriality property over the category of groups is transparent. 
It can also be generalized to the case in which $G$ is an infinite discrete group. However, it is not evident how to define a ring structure on this complex, and its physical relation to Ramond-Ramond fields is not clear. We include its definition here for completeness. ${ }^{9}$ See Appendix A and ref. [23] for the relevant definitions concerning modules over a functor category and their tensor products.

Starting from the real representation ring functor $\underline{\mathbb{R}}(-)=\mathbb{R} \otimes \underline{R}(-)$ over the orbit category $\operatorname{Or}(G)$, there is a natural map of real vector spaces

$$
\underline{\mathbb{R}}(-) \otimes_{\mathbb{R} O r}(G) \underline{C}^{\bullet}(X ; \mathbb{R}) \longrightarrow \operatorname{Hom}_{\mathbb{R} O r(G)}(\underline{C} \bullet(X ; \mathbb{R}), \underline{\mathbb{R}}(-)),
$$

where $\underline{C}^{\bullet}(X ; \mathbb{R})$ is the left $\mathbb{R} \operatorname{Or}(G)$-module obtained by dualizing the functor

$$
\underline{C} \bullet(X ; \mathbb{R}):=\mathbb{R} \otimes \underline{C} \bullet(X)
$$

defined in Sect. 2.2. Note that both $\underline{C} \bullet(X ; \mathbb{R})$ and $\underline{\mathbb{R}}(-)$, being contravariant functors, are right $\mathbb{R} \operatorname{Or}(G)$-modules. The map (5.8) is given on decomposable elements as

$$
\lambda \otimes f \longmapsto\left(\sigma \mapsto f(\sigma)_{*}(\lambda)\right)
$$

and it is an isomorphism of real vector spaces. ${ }^{10}$

Define the differential complex

$$
\Omega_{G}^{\bullet}(X ; \mathbb{R} \otimes \underline{R}(-)):=\underline{\mathbb{R}}(-) \otimes_{\mathbb{R} O r}(G) \underline{\Omega}^{\bullet}(X ; \mathbb{R}),
$$

where $\Omega^{\bullet}(X ; \mathbb{R})$ is the functor $\operatorname{Or}(G) \rightarrow \operatorname{Ab}$ given by $\underline{\Omega}^{\bullet}(X ; \mathbb{R}): G / H \mapsto \Omega^{\bullet}\left(X^{H} ; \mathbb{R}\right)$, and with derivation $\mathrm{d}_{\text {orb }}$ induced by the exterior derivative $\mathrm{d}$. Since the de Rham map induces a chain homotopy equivalence of left $\mathbb{R O r}(G)$-complexes $\underline{C^{\bullet}}(X ; \mathbb{R}) \rightarrow \underline{\Omega}^{\bullet}$ $(X ; \mathbb{R})$, there is a $G$-equivariant chain homotopy equivalence

$$
\underline{\mathbb{R}}(-) \otimes_{\mathbb{R O r}(G)} \underline{C}^{\bullet}(X ; \mathbb{R}) \longrightarrow \underline{\mathbb{R}}(-) \otimes_{\mathbb{R O r}(G)} \underline{\Omega}^{\bullet}(X ; \mathbb{R}) .
$$

Combined with the isomorphism (5.8) we can thus conclude

$$
\mathrm{H}_{G}^{\bullet}(X ; \mathbb{R} \otimes \underline{R}(-)) \cong \mathrm{H}\left(\Omega_{G}^{\bullet}(X ; \mathbb{R} \otimes \underline{R}(-)), \mathrm{d}_{\text {orb }}\right) .
$$

If one chooses to work with this complex, then the construction of orbifold differential K-theory groups given in Sect. 5.4 below can be carried through in exactly the same way. But since the two complexes $\Omega_{G}^{\bullet}(X ; \mathbb{R})$ and $\Omega_{G}^{\bullet}(X ; \mathbb{R} \otimes \underline{R}(-))$ are in general not isomorphic, the two differential cohomology theories obtained will be generically distinct.

5.4. Orbifold differential K-groups. Having sorted out all the ingredients necessary to make sense of a generalization of the diagram (5.2), we will now define the differential equivariant K-theory groups $\breve{\mathrm{K}}_{G}^{-n}(X)$. First, let us recall some further basic facts about equivariant K-theory. Similarly to ordinary K-theory, a model for the classifying space of the functor $\mathrm{K}_{G}^{0}$ is given by the $G$-algebra of Fredholm operators Fred ${ }_{G}$ acting on a separable Hilbert space which is a representation space for $G$ in which each irreducible representation occurs with infinite multiplicity [3]. Then there is an isomorphism

$$
\mathrm{K}_{G}^{0}(X) \cong\left[X, \text { Fred }_{G}\right]_{G},
$$

\footnotetext{
9 We are grateful to W. Lück for suggesting this construction to us.

10 In general, to have an isomorphism one has to require the $G$-manifold $X$ to be cocompact and proper.
} 
where $[-,-]_{G}$ denotes the set of equivalence classes of $G$-homotopic maps, and the isomorphism is given by taking the index bundle.

There is also a universal space $\operatorname{Vect}_{G}^{n}$, equipped with a universal $G$-bundle $\widetilde{E}_{G}^{n}$, such that $\left[X, \operatorname{Vect}_{G}^{n}\right]_{G}$ corresponds to the set of isomorphism classes of $n$-dimensional $G$-vector bundles over $X$ [46]. These spaces are constructed as follows. Let $\mathrm{E} G$ be the category whose objects are the elements of $G$ and with exactly one morphism between each pair of objects. The geometric realization (or nerve) of the set of isomorphism classes in $\mathrm{E} G$ is, as a simplicial space, the total space of the classifying principal $G$-bundle $E G$. With $\operatorname{Vect}^{n}(\mathrm{pt})$ the category of $n$-dimensional complex vector spaces $V \cong \mathbb{C}^{n}$, the universal space $\operatorname{Vect}_{G}^{n}$ is defined to be the geometric realization of the functor category $\left[\mathrm{E} G, \operatorname{Vect}^{n}(\mathrm{pt})\right]$ (see Appendix A). The universal $n$-dimensional $G$-vector bundle $\widetilde{E}_{G}^{n}$ is then defined as

$$
\widetilde{E}_{G}^{n}={\widetilde{\operatorname{Vect}_{G}^{n}}}^{\mathrm{GL}(n, \mathbb{C})} \mathbb{C}^{n} \longrightarrow \operatorname{Vect}_{G}^{n},
$$

where $\widetilde{\operatorname{Vect}}_{G}^{n}$ is the geometric realization of the functor category defined as above but with $\operatorname{Vect}^{n}(\mathrm{pt})$ replaced with the category consisting of objects $V$ in $\operatorname{Vect}^{n}(\mathrm{pt})$ together with an oriented basis of $V$.

We assume sufficient regularity conditions on the infinite-dimensional spaces Fred $_{G}$ and $\widetilde{E}_{G}^{n}$. Since Fred $_{G}$ and the group completion $\Omega B$ Vect $_{G}$ are both classifying spaces for equivariant K-theory, they are $G$-homotopic and we can thereby choose a cocycle

$$
u_{G} \in Z_{G}^{\text {even }}\left(\operatorname{Fred}_{G} ; \mathbb{R}\right)
$$

representing the equivariant Chern character of the universal $G$-bundle (5.9). Generally, the group $Z_{G}^{\text {even }}(X ; \mathbb{R})$ is the subgroup of closed cocycles in the complex

$$
C_{G}^{\text {even }}(X ; \mathbb{R}):=\bigoplus_{[g] \in G^{\vee}} C^{\text {even }}\left(X^{g} ; \mathbb{R}\right)^{Z_{G}(g)}
$$

which, by the results of Sect. 4.2, is a cochain model for the Bredon cohomology group $\mathrm{H}_{G}^{\text {even }-1}(X ; \mathbb{R} \otimes \underline{R}(-))$. The equivariant Chern character is understood to be composed with the delocalizing isomorphism of Sect. 4.2. Since it is a natural homomorphism, for any $G$-bundle $E \rightarrow X$ classified by a $G$-map $f: X \rightarrow$ Fred $_{G}$ one has

$$
\operatorname{ch}_{X}([E])=\left[f^{*} u_{G}\right] .
$$

Definition 5.1. The orbifold differential K-theory $\check{\mathbf{K}}_{G}^{0}(X)$ of the (global) orbifold $[X / G]$ is the group of triples $(c, h, \omega)$, where $c: X \rightarrow$ Fred $_{G}$ is a $G$-map, $\omega$ is an element in $\Omega_{G, \mathrm{cl}}^{\mathrm{even}}(X ; \mathbb{R})$, and $h$ is an element in $C_{G}^{\mathrm{even}-1}(X ; \mathbb{R})$ satisfying

$$
\delta h=\omega-c^{*} u_{G} .
$$

Two triples $\left(c_{0}, h_{0}, \omega_{0}\right)$ and $\left(c_{1}, h_{1}, \omega_{1}\right)$ are said to be equivalent if there exists a triple $(c, h, \omega)$ on $X \times[0,1]$, with the group $G$ acting trivially on the interval $[0,1]$ and with $\omega$ constant along $[0,1]$, such that

$$
\left.(c, h, \omega)\right|_{t=0}==\left(c_{0}, h_{0}, \omega_{0}\right) \quad \text { and }\left.\quad(c, h, \omega)\right|_{t=1}==\left(c_{1}, h_{1}, \omega_{1}\right) .
$$


In Eq. (5.11) the closed orbifold differential form $\omega$ is regarded as an orbifold cochain in the complex (5.10) by applying the de Rham map componentwise on the fixed point submanifolds $X^{g}, g \in G$. The higher orbifold differential K-theory groups $\check{\mathrm{K}}_{G}^{-n}(X)$ are defined analogously to those of Sect. 5.2 above. To confirm that this is an appropriate extension of the ordinary differential K-theory of $X$, we should show that the orbifold differential K-theory groups fit into exact sequences which reduce to those given by Eq. (5.7) when $G$ is taken to be the trivial group. For this, we define the group

$$
A_{\mathrm{K}_{G}}^{0}(X):=\left\{(\xi, \omega) \in \mathrm{K}_{G}^{0}(X) \times \Omega_{G, \mathrm{cl}}^{\mathrm{even}}(X ; \mathbb{R}) \mid \operatorname{ch}_{X}(\xi)=[\omega]_{G-\mathrm{dR}}\right\} .
$$

Theorem 5.2. The orbifold differential $K$-theory group $\check{\mathrm{K}}_{G}^{0}(X)$ satisfies the exact sequence

$$
0 \longrightarrow \frac{\mathrm{H}_{G}^{\text {even }-1}(X ; \mathbb{R} \otimes \underline{R}(-))}{\operatorname{ch}_{X}\left(\mathrm{~K}_{G}^{-1}(X)\right)} \longrightarrow \check{\mathrm{K}}_{G}^{0}(X) \longrightarrow A_{\mathrm{K}_{G}}^{0}(X) \longrightarrow 0 .
$$

Proof. Consider the subgroup of $\mathrm{H}_{G}^{\text {even-1}}(X ; \mathbb{R} \otimes \underline{R}(-))$ defined as the image of the equivariant $\mathrm{K}$-theory group $\mathrm{K}_{G}^{-1}(X)$ under the Chern character $\mathrm{ch}_{X}$. It consists of Bredon cohomology classes of the form $\left[\tilde{c}^{*} u_{G}^{-1}\right]$, where $\tilde{c}: X \rightarrow \Omega$ Fred $_{G}$. There is a surjective map

$$
\begin{aligned}
f: \check{\mathrm{K}}_{G}^{0}(X) & \longrightarrow A_{\mathrm{K}_{G}}^{0}(X), \\
{[(c, h, \omega)] } & \longmapsto([c], \omega),
\end{aligned}
$$

which is a well-defined homomorphism, i.e., it does not depend on the chosen representative of the orbifold differential K-theory class. By definition, the kernel of $f$ consists of triples of the form $(\underline{c}, h, 0)$. We also define the map

$$
\begin{aligned}
g: \mathrm{H}_{G}^{\mathrm{even}-1}(X ; \mathbb{R} \otimes \underline{R}(-)) & \longrightarrow \check{\mathrm{K}}_{G}^{0}(X) \\
{[h] } & \longmapsto[(\underline{c}, h, 0)],
\end{aligned}
$$

which is a well-defined homomorphism because the class $[(\underline{c}, h, 0)]$ depends only on the Bredon cohomology class $[h] \in \mathrm{H}_{G}^{\text {even-1 }}(X ; \mathbb{R} \otimes \underline{R}(-))$. Then by construction one has $\operatorname{im}(g)=\operatorname{ker}(f)$.

The homomorphism $g$ is not injective. To determine the kernel of $g$, we use the fact that the zero element in $\check{\mathrm{K}}_{G}^{0}(X)$ can be represented as

$$
[(\underline{c}, 0,0)]=\left[\left(\underline{c}, \pi_{*} F^{*} u_{G}+\mathrm{d}_{G} \sigma, 0\right)\right]
$$

with $F: X \times \mathbb{S}^{1} \rightarrow$ Fred $_{G}$ and $\sigma \in \Omega_{G}^{\text {even-2 }}(X ; \mathbb{R})$ (see Eq. (5.6)). To the map $F$ we can associate a map $\tilde{c}: X \rightarrow \Omega$ Fred $_{G}$ such that $F=\mathrm{ev} \circ\left(\tilde{c} \times \mathrm{id}_{\mathbb{S}^{1}}\right)$. This follows from the isomorphism

$$
\mathrm{K}_{G}^{-1}(X) \cong \operatorname{ker}\left(i^{*}: \mathrm{K}_{G}^{0}\left(X \times \mathbb{S}^{1}\right) \rightarrow \mathrm{K}_{G}^{0}(X)\right),
$$

where $i$ is the inclusion $i: X \hookrightarrow X \times \mathrm{pt} \subset X \times \mathbb{S}^{1}$. Now use the fact that at the level of (real) Bredon cohomology one has an equality

$$
\pi_{*}\left(\tilde{c} \times \mathrm{id}_{\mathbb{S}^{1}}\right)^{*}=\tilde{c}^{*} \Pi_{*},
$$


since the projection homomorphisms $\pi_{*}$ and $\Pi_{*}$ both correspond to integration (slant product) along the $\mathbb{S}^{1}$ fibre. Then one has the identity

$$
\left[\pi_{*} F^{*} u_{G}\right]==\left[\pi_{*}\left(\tilde{c} \times \operatorname{id}_{\mathbb{S}^{1}}\right)^{*} \mathrm{ev}^{*} u_{G}\right]==\left[\tilde{c}^{*} \Pi_{*} \mathrm{ev}^{*} u_{G}\right]==\left[\tilde{c}^{*} u_{G}^{-1}\right] .
$$

It follows that $\operatorname{ker}(g)$ is exactly the group $\operatorname{ch}_{X}\left(\mathrm{~K}_{G}^{-1}(X)\right)$, and putting everything together we arrive at Eq. (5.12).

The torus $\mathrm{H}_{G}^{\text {even-1 }}(X ; \mathbb{R} \otimes \underline{R}(-)) / \operatorname{ch}_{X}\left(\mathrm{~K}_{G}^{-1}(X)\right) \cong \mathrm{K}_{G}^{-1}(X) \otimes \mathbb{R} / \mathbb{Z}$ is called the group of topologically trivial flat fields (or of "orbifold Wilson lines"). We can rewrite the sequence (5.12) in various illuminating ways. Consider the characteristic class map

$$
\begin{aligned}
f_{\mathrm{cc}}: \check{\mathrm{K}}_{G}^{0}(X) & \longrightarrow \mathrm{K}_{G}^{0}(X) \\
{[(c, h, \omega)] } & \longmapsto[c]
\end{aligned}
$$

and the map

$$
\begin{aligned}
g_{\mathrm{cc}}: \Omega_{G}^{\mathrm{even}-1}(X ; \mathbb{R}) & \longrightarrow \check{\mathrm{K}}_{G}^{0}(X) \\
h & \longmapsto\left[\left(\underline{c}, h, \mathrm{~d}_{G} h\right)\right] .
\end{aligned}
$$

Let $\Omega_{\mathrm{K}_{G}}^{\text {even-1 }}(X ; \mathbb{R})$ be the subgroup of elements in $\Omega_{G, \mathrm{cl}}^{\text {even }-1}(X ; \mathbb{R})$ whose Bredon cohomology class lies in $\operatorname{ch}_{X}\left(\mathrm{~K}_{G}^{-1}(X)\right)$. Then by using arguments similar to those used in arriving at the sequence (5.12), one finds the

Corollary 5.3 (Characteristic class exact sequence). The orbifold differential $K$-theory group $\check{\mathrm{K}}_{G}^{0}(X)$ satisfies the exact sequence

$$
0 \longrightarrow \frac{\Omega_{G}^{\text {even-1}}(X ; \mathbb{R})}{\Omega_{\mathrm{K}_{G}}^{\text {even }-1}(X ; \mathbb{R})} \longrightarrow \check{\mathrm{K}}_{G}^{0}(X) \longrightarrow \mathrm{K}_{G}^{0}(X) \longrightarrow 0 .
$$

The quotient space of orbifold differential forms in the exact sequence (5.13) is called the group of topologically trivial fields. An element of this group is a globally defined (and hence topologically trivial) gauge potential on the orbifold $X / G$ up to large (quantized) gauge transformations, with $\omega$ the corresponding field strength. Finally, consider the field strength map

$$
\begin{aligned}
f_{\mathrm{fs}}: \check{\mathrm{K}}_{G}^{0}(X) & \longrightarrow \Omega_{G, \mathrm{cl}}^{\mathrm{even}}(X ; \mathbb{R}) \\
{[(c, h, \omega)] } & \longmapsto \omega .
\end{aligned}
$$

The kernel of the homomorphism $f_{\mathrm{fs}}$ is the group which classifies the flat fields (which are not necessarily topologically trivial) and is denoted $\mathrm{K}_{G}^{-1}(X ; \mathbb{R} / \mathbb{Z})$. This group will be described in more detail in the next section, where we shall also conjecture an essentially purely algebraic definition of $\mathrm{K}_{G}^{-1}(X ; \mathbb{R} / \mathbb{Z})$ which explains the notation. In any case, we have the

Corollary 5.4 (Field strength exact sequence). The orbifold differential $K$-theory group $\check{\mathrm{K}}_{G}^{0}(X)$ satisfies the exact sequence

$$
0 \longrightarrow \mathrm{K}_{G}^{-1}(X ; \mathbb{R} / \mathbb{Z}) \longrightarrow \check{\mathrm{K}}_{G}^{0}(X) \longrightarrow \Omega_{\mathrm{K}_{G}}^{\text {even }}(X ; \mathbb{R}) \longrightarrow 0 .
$$


Higher orbifold differential K-theory groups satisfy analogous exact sequences, with the appropriate degree shifts throughout. It is clear from our definition that one recovers the ordinary differential K-theory groups in the case of the trivial group $G=e$, and in this sense our orbifold differential K-theory may be regarded as its equivariant generalization. At this point we hasten to add that, although our groups are well-defined and satisfy desired properties which are useful for physical applications such as functoriality and the various exact sequences above, we have not proven that our orbifold theory is a differential cohomology theory. We have also not given a definition of what a generic orbifold (or equivariant) differential cohomology theory is. For instance, it would be interesting to define a ring structure and an integration on $\check{\mathrm{K}}_{G}^{\bullet}(X)$. In particular, the integration requires knowledge of a relative version of orbifold differential K-theory, which we have not developed in this paper.

We have also investigated the possibility that the group $\check{\mathrm{K}}_{G}^{\bullet}(X)$ reduces to the ordinary differential K-theory $\check{\mathrm{K}}^{\bullet}(X / G)$ in the case of a free $G$-action on $X$, and to $\check{\mathrm{K}}^{\bullet}(X) \otimes R(G)$ in the case of a trivial group action, as one might naively expect from the analogous results for equivariant topological K-theory (the equivariant excision theorem (1.4) with $N=G$ and Eq. (3.4), respectively) and for Bredon cohomology (Examples 2.2 and 2.3, respectively). On the contrary, these decompositions do not occur, because the corresponding isomorphisms in equivariant K-theory are estabilished via the induction maps and these usually do not lift at the "cochain level" as isomorphisms. Properties such as induction structures reflect homotopy invariance of topological cohomology groups, which is not possessed by differential cohomology groups due to their "local" dependence on the complex of differential forms. We will see an explicit example of this in the next section. With this in mind, it would be interesting then to define a suitable analog of the induction structures in an equivariant cohomology theory. These and various other interesting mathematical issues surrounding the orbifold differential K-theory groups that we have defined will not be pursued in this paper.

\section{Flux Quantization of Orbifold Ramond-Ramond Fields}

In this final section we will argue that the orbifold differential K-theory defined in the previous section can be used to describe Ramond-Ramond fields and their flux quantization condition in orbifolds of Type II superstring theory with vanishing $H$-flux. To formulate the self-duality property of orbifold Ramond-Ramond fields in equivariant K-theory, one needs an appropriate equivariant version of Pontrjagin duality [32]. This appears to be a very deep and complicated problem, and is beyond the scope of the present paper. Furthermore, to generalize the pairing of Sect. 4.4 to topologically non-trivial Ramond-Ramond fields, one needs to define an integration on the orbifold differential cohomology theory defined in Sect. 5.4, and regard the Ramond-Ramond fields properly as cocycles for it. In addition, one needs a graded ring structure and an appropriate groupoid representing the orbifold differential K-theory, whose objects are the Ramond-Ramond form gauge potentials $C$ and whose isomorphism classes are the gauge equivalence classes in $\check{\mathrm{K}}_{G}^{\bullet}(X)$. Lacking these ingredients, most of our analysis in this section will be essentially purely "topological". We shall study the somewhat simpler problem of the proper K-theory quantization of orbifold Ramond-Ramond fields, in particular due to their sourcing by fractional D-branes, in terms of the formulation provided by orbifold differential K-theory. 
6.1. Ramond-Ramond currents. We will begin by rephrasing the relation between the D-brane charge group and the group of Ramond-Ramond fluxes "measured at infinity" in the equivariant case, which is a statement about the K-theoretic classification of Ramond-Ramond fields on a global orbifold $[X / G]$. For this, we invoke an argument due to Moore and Witten [52] which will suggest that the equivariant Chern character $\mathrm{ch}_{X}$ constructed in Sect. 2 gives the right quantization rule for orbifold Ramond-Ramond fields. Suppose that our spacetime $X$ is a non-compact $G$-manifold. Suppose further that there are D-branes present in Type II superstring theory on $X / G$. Their RamondRamond charges are classified by the equivariant K-theory $\mathrm{K}_{G, \mathrm{cpt}}^{i}(X)$ with compact support, where $i=0$ in Type IIB theory and $i=-1$ in Type IIA theory.

We require that the brane be a source for the equation of motion for the total Ramond-Ramond field strength $\omega$. This means that it creates a Ramond-Ramond current $J$. If we require that the worldvolume $W$ be compact in equivariant K-cycles $(W, E, f) \in \mathrm{D}^{G}(X)$, then $J$ is supported in the interior ${ }_{X}^{\circ}$ of $X$. Let $X_{\infty}$ be the "boundary of $X$ at infinity", which we assume is preserved by the action of $G$. Then $\mathrm{K}_{G, \text { cpt }}^{\bullet}(X) \cong \mathrm{K}_{G}^{\bullet}\left(X, X_{\infty}\right)$. Since $J$ is trivialized by $\omega$ in $\stackrel{\circ}{X}$, the D-brane charge lives in the kernel of the natural forgetful homomorphism

$$
\mathfrak{f}^{\bullet}: \mathrm{K}_{G, \mathrm{cpt}}^{\bullet}(X) \longrightarrow \mathrm{K}_{G}^{\bullet}(X)
$$

induced by the inclusion $(X, \emptyset) \hookrightarrow\left(X, X_{\infty}\right)$. We denote by $i: X_{\infty} \hookrightarrow X$ the canonical inclusion.

The long exact sequence for the pair $\left(X, X_{\infty}\right)$ in equivariant K-theory truncates, by Bott periodicity, to the six-term exact sequence

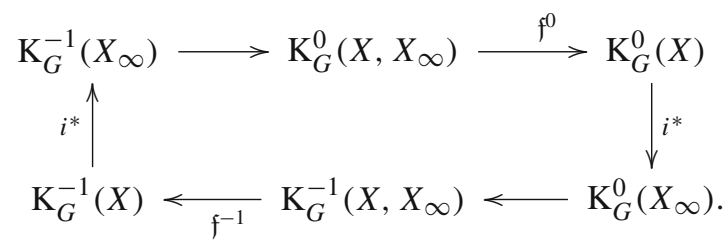

It follows that the charge groups are given by

$$
\operatorname{ker}\left(\mathfrak{f}^{0}\right) \cong \frac{\mathrm{K}_{G}^{-1}\left(X_{\infty}\right)}{i^{*}\left(\mathrm{~K}_{G}^{-1}(X)\right)} \quad \text { and } \quad \operatorname{ker}\left(\mathfrak{f}^{-1}\right) \cong \frac{\mathrm{K}_{G}^{0}\left(X_{\infty}\right)}{i^{*}\left(\mathrm{~K}_{G}^{0}(X)\right)}
$$

This formula means that the group of Type IIB (resp. Type IIA) brane charges is measured by the group $\mathrm{K}_{G}^{-1}\left(X_{\infty}\right)$ (resp. $\mathrm{K}_{G}^{0}\left(X_{\infty}\right)$ ) of "orbifold Ramond-Ramond fluxes at infinity" which cannot be extended to all of spacetime $X$. We may then interpret, for arbitrary spacetimes $X$, the group $\mathrm{K}_{G}^{-1}(X)$ (resp. $\left.\mathrm{K}_{G}^{0}(X)\right)$ as the group classifying Ramond-Ramond fields in the orbifold $X / G$ which are not sourced by branes in Type IIB (resp. Type IIA) string theory.

The Ramond-Ramond current can be described explicitly in the delocalized theory of Sect. 4. The Wess-Zumino pairing (4.9) between a topologically trivial, complex Ramond-Ramond potential and a D-brane represented by an equivariant K-cycle $(W, E, f) \in \mathrm{D}^{G}(X)$ contributes a source term to the Ramond-Ramond equations of motion, which is the class

$$
[Q(W, E, f)] \in \mathrm{H}_{G}^{\mathrm{even}}(X ; \mathbb{C} \otimes \underline{R}(-))
$$


represented by the pushforward

$$
Q(W, E, f)=f_{!}^{\mathrm{H}_{G}}\left(\operatorname{ch}^{\mathbb{C}}(E) \wedge_{G} \mathcal{R}(W, f)\right) .
$$

We now use the Riemann-Roch formula (4.17) and the fact that $f^{*}$ is right adjoint to $f_{!}^{\mathrm{H}_{G}}$, i.e., $f_{!}^{\mathrm{H}_{G}} \circ f^{*}=\mathrm{id}_{\mathrm{H}_{G}^{\bullet}}(X ; \underline{\mathbb{C}}(-))$. Using the explicit expression for the curvature form in Eq. (4.21), we can then rewrite this class as

$$
Q(W, E, f)=\operatorname{ch}^{\mathbb{C}}\left(f_{!}^{\mathrm{K}_{G}}(E)\right) \wedge_{G} \sqrt{\operatorname{Todd}_{G}\left(T_{X}\right) \wedge_{G} \Lambda_{G}(X)}
$$

This is the complex Bredon cohomology class of the Ramond-Ramond current $J$ created by the D-brane $(W, E, f)$. In the case $G=e$, the expression (6.2) reduces to the standard class of the current for Ramond-Ramond fields in Type II superstring theory on $X[19,49,52,54]$.

There is a natural extension of the current (6.2) which allows us to formally conclude, in analogy with the non-equivariant case, that the complex Bredon cohomology class associated to a class $\xi \in \mathrm{K}_{G}^{\bullet}(X) \otimes \mathbb{C}$ representing a Ramond-Ramond field is assigned by the equivariant Chern character. If the Ramond-Ramond field is determined by a differential form $C / 2 \pi \sqrt{\operatorname{Todd}_{G}\left(T_{X}\right) \wedge_{G} \Lambda_{G}(X)}$ with $C \in \Omega_{G}^{\bullet}(X ; \mathbb{C})$ and $\mathrm{d}_{G} C=\omega$, then this is the class $[\omega(\xi)]$ in $\mathrm{H}_{G}^{\bullet}(X ; \mathbb{C} \otimes \underline{R}(-))$ represented by the closed differential form

$$
\frac{\omega(\xi)}{2 \pi \sqrt{\operatorname{Todd}_{G}\left(T_{X}\right) \wedge_{G} \Lambda_{G}(X)}}=\operatorname{ch}^{\mathbb{C}}(\xi) .
$$

This is just the anticipated flux quantization condition from orbifold differential K-theory. The appearance of the additional gravitational terms in Eq. (6.3) is inconsequential to this identification. Given the canonical map (5.1) in a generalized cohomology theory $\mathrm{E}^{\bullet}$, any other $\operatorname{map} \mathrm{E}^{\bullet}(X) \rightarrow \mathrm{H}\left(X ; \mathbb{R} \otimes \pi_{-} \bullet \mathrm{E}^{\bullet}\right.$ with the same properties described in Sect. 5.1 is obtained by multiplying $\varphi$ with an invertible element in $\mathrm{H}\left(X ; \mathbb{R} \otimes \pi_{-\bullet} \mathrm{E}\right)^{0}$. In the case at hand, the characteristic class $\sqrt{\operatorname{Todd}_{G}\left(T_{X}\right) \wedge_{G} \Lambda_{G}(X)}$ is an invertible closed differential form which represents this element in $\mathrm{H}_{G}^{\text {even }}(X ; \mathbb{C} \otimes \underline{R}(-))$. This class reduces to the usual gravitational correction $\sqrt{\operatorname{Todd}\left(T_{X}\right)}$ when $G$ acts trivially on $X$.

We should stress that this analysis of the delocalized theory assumes the strong conditions spelled out in Sect. 4.7, which require a deep geometrical compatibility of the equivariant $\mathrm{K}$-cycle $(W, E, f)$ with the orbifold structure of $[X / G]$ (or else an explicit determination of the unknown characteristic class $\Lambda_{G}(W)$ correcting the Riemann-Roch formula as explained in Sect. 4.6). The example of the linear orbifolds considered in Sects. 3.3 and 4.5, and in Section 6.2 below, is simple enough to satisfy these conditions. It would be very interesting to find a geometrically non-trivial explicit example to test these requirements on. In any case, the results above suggest that the orbifold differential $\mathrm{K}$-theory (or more precisely a complex version of it) defined in the previous section is the natural framework in which to describe topologically non-trivial Ramond-Ramond fields on orbifolds. It would be highly desirable to determine the correct generalization of Eq. (6.2) to the orbifold differential K-theory group $\check{\mathrm{K}}_{G}^{\bullet}(X)$ of the previous section, and thereby extending the delocalized Ramond-Ramond currents to include effects such as torsion. 
6.2. Linear orbifolds. To understand certain aspects of the orbifold differential K-theory groups, it is instructive to study the K-theory classification of Ramond-Ramond fields on the linear orbifolds considered in Sects. 3.3 and 4.5. Since the $\mathbb{C}$-linear $G$-module $V$ is equivariantly contractible, one has $\mathrm{H}_{G}^{\text {odd }}(V ; \mathbb{R} \otimes \underline{R}(-))=0$ and $\mathrm{K}_{G}^{0}(V)=R(G)$. From Theorem 5.2 it then follows that

$$
\check{\mathrm{K}}_{G}^{0}(V) \cong A_{\mathrm{K}_{G}}^{0}(X) \cong\left\{(\gamma, \omega) \in R(G) \times \Omega_{G, \mathrm{cl}}^{\mathrm{even}}(V ; \mathbb{R}) \mid \operatorname{ch}_{G / G}(\gamma)=[\omega]_{G-\mathrm{dR}}\right\} .
$$

Since the equivariant Chern character $\operatorname{ch}_{G / H}: R(H) \rightarrow R(H)$ for $H \leq G$ is the identity map, the setwise fibre product truncates to the lattice of quantized orbifold differential forms and one has

$$
\check{\mathrm{K}}_{G}^{0}(V)=\Omega_{\mathrm{K}_{G}}^{\mathrm{even}}(V ; \mathbb{R}) .
$$

This is the group of Type IIA Ramond-Ramond form potentials on $V$. It naturally contains those fields which trivialize the Ramond-Ramond currents sourced by the stable fractional D0-branes of the Type IIA theory, corresponding to characteristic classes $[c]$ in the representation ring $R(G)$ as explained in Sect. 3.3.

This can be explicitly described as an extension of the group of topologically trivial Ramond-Ramond fields $C$ of odd degree by the equivariant K-theory of $V$, as implied by Corollary 5.3. Since $V$ is connected and $G$-contractible, one has $\Omega_{G, \mathrm{cl}}^{0}(V ; \mathbb{R})=$ $\mathbb{R} \otimes R(G)$ and the group (6.4) has a natural splitting

$$
\check{\mathrm{K}}_{G}^{0}(V)=R(G) \oplus\left(\bigoplus_{k=1}^{d} \Omega_{G, \mathrm{cl}}^{2 k}(V ; \mathbb{R})\right) .
$$

Any closed orbifold form $\omega$ on $V$ of positive degree is exact, $\omega=\mathrm{d}_{G} C$, with the gauge invariance $C \mapsto C+\mathrm{d}_{G} \xi$. It follows that there is a natural map

$$
\bigoplus_{k=1}^{d} \Omega_{G, \mathrm{cl}}^{2 k}(V ; \mathbb{R}) \longrightarrow \frac{\Omega_{G}^{\text {odd }}(V ; \mathbb{R})}{\Omega_{\mathrm{K}_{G}}^{\text {odd }}(V ; \mathbb{R})}
$$

which associates to the field strength $\omega$ the corresponding globally well-defined Ramond-Ramond potential $C$.

On the other hand, the orbifold differential K-theory group $\check{\mathrm{K}}_{G}^{-1}(V)$ of Type IIB Ramond-Ramond fields on $V$ can be computed by using the characteristic class exact sequence (5.13) with degree shifted by -1 . Using $\mathrm{K}_{G}^{-1}(V)=0$, one finds

$$
\check{\mathrm{K}}_{G}^{-1}(V)=\frac{\Omega_{G}^{\text {even }}(V ; \mathbb{R})}{\Omega_{\mathrm{K}_{G}}^{\text {even }}(V ; \mathbb{R})} .
$$

This result reflects the fact that the Type IIB theory has no stable fractional D0-branes. Hence there is no extension and the Ramond-Ramond fields are induced solely by the closed string background. Their field strengths $\omega=\mathrm{d}_{G} C$ are determined entirely by the potentials $C$, which are globally defined differential forms of even degree.

Note that for any $G$-homogeneous space $G / H$ one has $\mathrm{K}_{G}^{-1}(G / H)=0$ and

$$
\Omega_{G}^{\text {odd }}(G / H ; \mathbb{R})=0 .
$$


From the characteristic class exact sequence (5.13) one thus computes that the orbifold differential K-theory group

$$
\check{\mathrm{K}}_{G}^{0}(G / H) \cong \mathrm{K}_{G}^{0}(G / H) \cong R(H)
$$

is given by the characteristic classes (of fractional D0-branes), while Theorem 5.2 (with degree shifted by -1 ) implies that the orbifold differential K-theory group

$$
\check{\mathrm{K}}_{G}^{-1}(G / H) \cong \frac{\mathrm{H}_{G}^{\text {even }}(G / H ; \mathbb{R} \otimes \underline{R}(-))}{\operatorname{ch}_{G / H}\left(\mathrm{~K}_{G}^{0}(G / H)\right)} \cong R(H) \otimes \mathbb{R} / \mathbb{Z}
$$

is given by the topologically trivial flat fields. Setting $H=G$ in Eqs. (6.7) and (6.8) shows that the differential $\mathrm{K}_{G}$-theory groups of a point generically differ from the groups (6.4) and (6.6), even though $V$ is $G$-contractible. This exemplifies the $G$-homotopy non-invariance of the orbifold differential K-theory groups, required to capture the nonvanishing (but topologically trivial) gauge potentials on $V$.

6.3. Flat potentials. In Sect. 6.2 above we encountered some examples of topologically trivial Ramond-Ramond fields, corresponding to gauge equivalence classes with trivial K-theory flux $[c]=0$. They are the globally defined orbifold differential forms

$$
C \in \Omega_{G}^{\bullet}(X ; \mathbb{R})
$$

with the gauge symmetry $C \rightarrow C+\xi$, where $\mathrm{d}_{G} \xi=0$ and $\xi \in \Omega_{\mathrm{K}_{G}}^{\bullet}(X ; \mathbb{R})$, and field strength

$$
\omega=\mathrm{d}_{G} C .
$$

The flat Ramond-Ramond fields are instead classified by the abelian group $\mathrm{K}_{G}^{i}(X ; \mathbb{R} / \mathbb{Z})$, where $i=0$ for Type IIB theory and $i=-1$ for Type IIA theory. In the previous section this group was defined to be the subgroup of orbifold differential K-theory with vanishing curvature. In the following we will conjecture a very natural algebraic definition of these groups which ties them somewhat more directly to equivariant K-theory groups.

To motivate this conjecture, we first compute the groups $\mathrm{K}_{G}^{\bullet}(V ; \mathbb{R} / \mathbb{Z})$ for the linear orbifolds of Sect. 6.2 above, wherein the associated differential K-theory groups were determined explicitly. Using the field strength exact sequence (5.15), by definition one has

$$
\mathrm{K}_{G}^{-1}(V ; \mathbb{R} / \mathbb{Z}) \cong \operatorname{ker}\left(f_{\mathrm{fs}}: \check{\mathrm{K}}_{G}^{0}(V) \rightarrow \Omega_{\mathrm{K}_{G}}^{\mathrm{even}}(V ; \mathbb{R})\right)
$$

which from the natural isomorphism (6.4) trivially gives

$$
\mathrm{K}_{G}^{-1}(V ; \mathbb{R} / \mathbb{Z})=0 .
$$

Similarly, using $\mathrm{K}_{G}^{-1}(V)=0$ one has

$$
\mathrm{K}_{G}^{0}(V ; \mathbb{R} / \mathbb{Z}) \cong \operatorname{ker}\left(f_{\mathrm{fs}}: \check{\mathrm{K}}_{G}^{-1}(V) \rightarrow \Omega_{G, \mathrm{cl}}^{\mathrm{odd}}(V ; \mathbb{R})\right)
$$

Using the natural isomorphism (6.6), the field strength map is

$$
f_{\mathrm{fs}}([C])=\mathrm{d}_{G} C \quad \text { for } C \in \Omega_{G}^{\mathrm{even}}(V ; \mathbb{R}),
$$


giving

$$
\mathrm{K}_{G}^{0}(V ; \mathbb{R} / \mathbb{Z}) \cong \frac{\Omega_{G, \mathrm{cl}}^{\text {even }}(V ; \mathbb{R})}{\Omega_{\mathrm{K}_{G}}^{\text {even }}(V ; \mathbb{R})} .
$$

Similarly to Eq. (6.5), there is a natural splitting of the vector space of closed orbifold differential forms given by

$$
\Omega_{G, \mathrm{cl}}^{\mathrm{even}}(V ; \mathbb{R})=(R(G) \otimes \mathbb{R}) \oplus\left(\bigoplus_{k=1}^{d} \Omega_{G, \mathrm{cl}}^{2 k}(V ; \mathbb{R})\right)
$$

and we arrive finally at

$$
\mathrm{K}_{G}^{0}(V ; \mathbb{R} / \mathbb{Z})=R(G) \otimes \mathbb{R} / \mathbb{Z} .
$$

These results of course simply follow from the fact that $V$ is $G$-contractible, so that every $\mathrm{d}_{G}$-closed Ramond-Ramond field is trivial, except in degree zero where the gauge equivalence classes are naturally parametrized by the twisted sectors of the string theory in Eq. (6.10). Note that both groups of flat fields (6.9) and (6.10) are unchanged by (equivariant) contraction of the $G$-module $V$ to a point, as an analogous (but simpler) calculation shows. This suggests that the groups $\mathrm{K}_{G}^{\bullet}(X ; \mathbb{R} / \mathbb{Z})$ have at least some $G$-homotopy invariance properties, unlike the differential $\mathrm{K}_{G}$-theory groups. This motivates the following conjectural algebraic framework for describing these groups.

We will propose that the group $\mathrm{K}_{G}^{\bullet}(X ; \mathbb{R} / \mathbb{Z})$ is an extension of the torus of topologically trivial flat orbifold Ramond-Ramond fields by the torsion elements in $\mathrm{K}_{G}^{\bullet+1}(X)$, as they have vanishing image under the equivariant Chern character $\operatorname{ch}_{X}$. The resulting group may be called the "equivariant K-theory with coefficients in $\mathbb{R} / \mathbb{Z}$ ". The short exact sequence of coefficient groups

$$
0 \longrightarrow \mathbb{Z} \longrightarrow \mathbb{R} \longrightarrow \mathbb{R} / \mathbb{Z} \longrightarrow 0
$$

induces a long exact sequence of equivariant K-theory groups which, by Bott periodicity, truncates to the six-term exact sequence

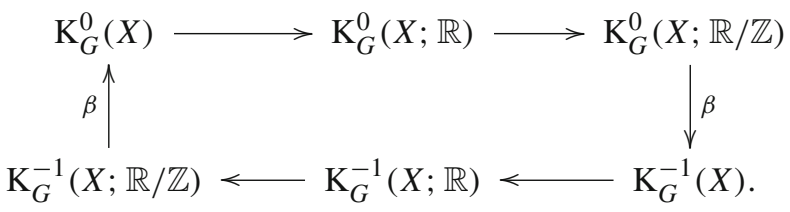

The connecting homomorphism $\beta$ is a suitable variant of the usual Bockstein homomorphism. We assume that the equivariant K-theory with real coefficients is defined simply by the $\mathbb{Z}_{2}$-graded ring

$$
\mathrm{K}_{G}^{\bullet}(X ; \mathbb{R})==\mathrm{K}_{G}^{\bullet}(X) \otimes \mathbb{R} \cong \mathrm{H}_{G}^{\bullet}(X ; \mathbb{R} \otimes \underline{R}(-)),
$$

where we have used Theorem 2.5. The maps to real K-theory in Eq. (6.11) may then be identified with the equivariant Chern character $\mathrm{ch}_{X}$, whose image is a full lattice in the Bredon cohomology group $\mathrm{H}_{G}^{\bullet}(X ; \mathbb{R} \otimes \underline{R}(-))$. Then the abelian group $\mathrm{K}_{G}^{\bullet}(X ; \mathbb{R} / \mathbb{Z})$ sits in the exact sequence

$$
0 \longrightarrow \mathrm{K}_{G}^{\bullet}(X) \otimes \mathbb{R} / \mathbb{Z} \longrightarrow \mathrm{K}_{G}^{\bullet}(X ; \mathbb{R} / \mathbb{Z}) \stackrel{\beta}{\rightarrow} \text { Tor }\left(\mathrm{K}_{G}^{\bullet+1}(X)\right) \longrightarrow 0 .
$$


When $G=e$, Eq. (6.11) is the usual Bockstein exact sequence for K-theory. In this case, an explicit geometric realization of the groups $\mathrm{K}^{\bullet}(X ; \mathbb{R} / \mathbb{Z})$ in terms of bundles with connection has been given by Lott [43]. Moreover, in ref. [37] a geometric construction of the map $\mathrm{K}^{-1}(X ; \mathbb{R} / \mathbb{Z}) \rightarrow \check{\mathrm{K}}^{0}(X)$ in the field strength exact sequence is given. Unfortunately, no such geometrical description is immediately available for our equivariant differential K-theory, due to the lack of a Chern-Weil theory for the homotopy theoretic equivariant Chern character of Sect. 2.4. Our conjectural definition (6.12) is satisfied by the linear orbifold groups (6.9) and (6.10).

In ref. [14] a very different definition of the groups $\mathrm{K}_{G}^{\bullet}(X ; \mathbb{R} / \mathbb{Z})$ is given, by defining both equivariant $\mathrm{K}$-theory and cohomology using the Borel construction of Example 1.3. Then the Bockstein exact sequence (6.11) is written for the ordinary K-theory groups of the homotopy quotient $X_{G}=E G \times_{G} X$. While these groups reduce, like ours, to the usual K-theory groups of flat fields when $G=e$, they do not obey the exact sequence (6.12). The reason is that the equivariant Chern character used is not an isomorphism over the reals, as explained in Sect. 2.1 (see also ref. [47] for a description of $\mathrm{K}^{\bullet}\left(X_{G}\right)$ as the completion of $\mathrm{K}_{G}^{\bullet}(X)$ with respect to a certain ideal). Moreover, an associated differential K-theory construction would directly involve differential forms on the infinite-dimensional space $X_{G}$ which is only homotopic to the finite-dimensional $\mathrm{CW}$-complex $X / G$. The physical interpretation of such fields is not clear. Even in the simple case of the linear orbifolds $V$ studied above, this description predicts an infinite set of equivariant fluxes of arbitrarily high dimension on the infinite-dimensional classifying space $B G$, and one must perform some non-canonical quotients in order to try to isolate the physical fluxes. The differences between the equivariant K-theory and Borel cohomology groups of $V$ also require postulating certain effects of fractional branes on the orbifold, as in ref. [12]. In contrast, with our constructions the relation between orbifold flux groups and Bredon cohomology is much more natural, and it involves only finitely-many orbifold Ramond-Ramond fields.

6.4. Consistency conditions. As we have stressed throughout this paper, the usage of Borel cohomology as a companion to equivariant K-theory in the topological classification of D-branes and Ramond-Ramond fluxes on orbifolds has various undesirable features, most notably the fact that it involves torsion classes substantially, especially when finite group cohomology is involved. In our applications to string geometry, it is more convenient to use an equivariant cohomology theory with substantial torsion-free information. Bredon cohomology naturally accomplishes this, as instead of group cohomology the basic object is a representation ring. In fact, as we now demonstrate, the formulation of topological consistency conditions for orbifold Ramond-Ramond fields and D-branes within the framework of equivariant K-theory naturally necessitates the use of classes in Bredon cohomology.

Given a Bredon cohomology class $\lambda \in \mathrm{H}_{G}^{\bullet}(X ; \underline{R}(-))$, let us ask if there exists a Ramond-Ramond field for which $\omega=\lambda$ in the sense of Eq. (6.3). For this, we must find an equivariant $\mathrm{K}$-theory lift $\xi \in \mathrm{K}_{G}^{\bullet}(X)$ of $\lambda$. As in the non-equivariant case $[22,48]$, the obstructions to such a lift can be determined via a suitable spectral sequence. For equivariant K-theory the appropriate spectral sequence is described in refs. [20,50] (see also ref. [58]) using the skeletal filtration $\left(X_{n}\right)$ of Sect. 1.1. We will now briefly explain the construction of this spectral sequence and its natural relationship with the obstruction theory for Ramond-Ramond fluxes in Bredon cohomology. 
The $\mathrm{E}_{1}$-term of the spectral sequence is the relative $G$-equivariant $\mathrm{K}$-theory group

$$
\mathrm{E}_{1}^{p, q}=\mathrm{K}_{G}^{p+q}\left(X_{p}, X_{p-1}\right)
$$

with differential

$$
\mathrm{d}_{1}^{p, q}: \mathrm{E}_{1}^{p, q} \longrightarrow \mathrm{E}_{1}^{p+1, q}
$$

induced by the long exact sequence of the triple $\left(X_{p+1}, X_{p}, X_{p-1}\right)$ in equivariant $\mathrm{K}$ theory, i.e., $\mathrm{d}_{1}^{p, q}$ is the composition of the map $i^{*}$ induced by the inclusion $i:\left(X_{p}, \emptyset\right) \hookrightarrow$ $\left(X_{p}, X_{p-1}\right)$ with the cellular coboundary operator of the pair $\left(X_{p+1}, X_{p}\right)$. From Eq. (1.1) it follows that there is a homeomorphism $\coprod_{j \in J_{p}}\left(\stackrel{\circ}{\mathbb{B}}_{j}^{p} \times G / K_{j}\right) \rightarrow X_{p} \backslash X_{p-1}$, and hence

$$
\mathrm{E}_{1}^{p, q} \cong \bigoplus_{j \in J_{p}} \mathrm{~K}_{G}^{p+q}\left(\stackrel{\circ}{B}_{j}^{p} \times G / K_{j}\right) \cong \bigoplus_{j \in J_{p}} \mathrm{~K}_{G}^{q}\left(G / K_{j}\right) .
$$

Thus $\mathrm{E}_{1}^{p, q}=0$ for $q$ odd, while for $q$ even the group $\mathrm{E}_{1}^{p, q}$ is a direct sum of representation rings $R\left(K_{j}\right)$ over all isotropy subgroups of $p$-cells of orbit type $G / K_{j}$. It parametrizes equivariant $\mathrm{K}$-theory classes defined on the $p$-skeleton of $X$ which are trivial on the $(p-1)$-skeleton, and gives the supports of $p$-form fields and charges on the orbifold which carry no lower or higher degree fluxes.

The $E_{2}$-term of the spectral sequence is the cohomology of the differential $d_{1}$. The cohomology of the cochain complex assembled from such terms is the equivariant cohomology with coefficient system $\underline{R}(-)$ on $\operatorname{Or}\left(G, \mathfrak{F}\left(X_{p}\right)\right)$ for $q=0$, and thus a necessary condition for a $p$-form Ramond-Ramond field to lift to $\mathrm{K}_{G}^{\bullet}(X)$ is that it define a non-trivial cocycle in Bredon cohomology. This is consistent with Definition 5.1. The resulting Atiyah-Hirzebruch spectral sequence may then be written

$$
\mathrm{E}_{2}^{p, q}=\mathrm{H}_{G}^{p}\left(X ; \underline{\pi-q}_{G}(-)\right) \Longrightarrow \mathrm{K}_{G}^{p+q}(X)
$$

and it lives in the first and fourth quadrants of the $(p, q)$-plane. On the $r^{\text {th }}$ terms $\mathrm{E}_{r}^{p, q}$, the differential $\mathrm{d}_{r}^{p, q}$ has bidegree $(r,-r+1)$, and $\mathrm{E}_{r+1}^{p, q}$ is the corresponding cohomology group. Note that $\mathrm{d}_{r}^{p, q}=0$ for all $r$ even, since then either its source or its target vanishes (as $\mathrm{K}^{q}(\mathbb{C}[H])=0$ for all $q$ odd and $\left.H \leq G\right)$. The $\mathrm{E}_{\infty}$-term is the inductive limit

$$
\mathrm{E}_{\infty}^{p, q}=\underset{r}{\lim } \mathrm{E}_{r}^{p, q} \text {. }
$$

For a finite-dimensional manifold $X$, one has $\mathrm{E}_{r}^{p, q}=\mathrm{E}_{\infty}^{p, q}$ for all $r>\operatorname{dim}(X)$ and the spectral sequence converges to $\mathrm{K}_{G}^{p+q}(X)$. This means that the $\mathrm{E}_{\infty}$-term is the associated graded group of a decreasing finite filtration filt ${ }_{p, q} \mathrm{~K}_{G}^{p+q}(X) \subset$ filt $_{p-1, q+1} \mathrm{~K}_{G}^{p+q}(X)$, $0 \leq p \leq \operatorname{dim}(X)$ with $\mathrm{K}_{G}^{q}(X)=$ filt $_{0, q} \mathrm{~K}_{G}^{q}(X)$ and

$$
\frac{\text { filt }_{p, q} \mathrm{~K}_{G}^{p+q}(X)}{\text { filt }_{p+1, q-1} \mathrm{~K}_{G}^{p+q}(X)} \cong \mathrm{E}_{\infty}^{p, q} .
$$

Explicitly, if $\iota: X_{p-1} \hookrightarrow X$ denotes the inclusion of the $(p-1)$-skeleton in $X$, then the filtration groups

$$
\text { filt }_{p, q} \mathrm{~K}_{G}^{p+q}(X):=\operatorname{ker}\left(\iota^{*}: \mathrm{K}_{G}^{p+q}(X) \rightarrow \mathrm{K}_{G}^{p+q}\left(X_{p-1}\right)\right)
$$


consist of Ramond-Ramond fluxes where the field strength $\omega$ is a form of degree $\geq p$, while the extension groups (6.13) consist of $p$-form fluxes with vanishing higher and lower degree fluxes. By Theorem 2.5, the equivariant Chern character $\operatorname{ch}_{X}$ determines an isomorphism from the limit of the spectral sequence to its $\mathrm{E}_{2}$-term. Thus the spectral sequence collapses rationally, and so the images of all higher differentials $\mathrm{d}_{r}^{p, q}, r>2$ in the spectral sequence consist of torsion classes.

It follows that the next non-trivial obstruction to extending a Ramond-Ramond field is given by a "cohomology operation"

$$
\mathrm{d}_{3}^{p, 0}: \mathrm{H}_{G}^{p}(X ; \underline{R}(-)) \longrightarrow \mathrm{H}_{G}^{p+3}(X ; \underline{R}(-)) .
$$

Thus a necessary condition for a Bredon cohomology class $\lambda \in \mathrm{H}_{G}^{p}(X ; \underline{R}(-))$ to survive to $\mathrm{E}_{\infty}^{p, 0}$ is given by

$$
\mathrm{d}_{3}^{p, 0}(\lambda)=0
$$

We interpret the condition (6.15) as a (partial) requirement of global worldsheet anomaly cancellation for Ramond-Ramond fluxes and, dually, the worldvolume homology cycles that they pair with. This is the orbifold generalization of the Freed-Witten condition $[22,30,48]$ formulated in terms of obstruction classes in Bredon cohomology. It is a necessary condition for the existence of a fractional D-brane whose lowest non-vanishing Ramond-Ramond charge is $\lambda \in \mathrm{H}_{G}^{p}(X ; \underline{R}(-))$. On the other hand, in computing the $E_{3}$-term as the cohomology of the differential (6.14), we must also take the quotient by the image of $\mathrm{d}_{3}^{p-3,0}$. This means that a class $\lambda$ satisfying Eq. (6.15) must be further subjected to the identifications

$$
\lambda \sim \lambda+\mathrm{d}_{3}^{p-3,0}\left(\lambda^{\prime}\right)
$$

in $\mathrm{E}_{3}^{p, 0}$, for any class $\lambda^{\prime} \in \mathrm{H}_{G}^{p-3}(X ; \underline{R}(-))$. We interpret the condition (6.16) as accounting for Ramond-Ramond charge violation due to D-instanton effects in the orbifold background, as explained in ref. [48] for the non-equivariant case. It means that while there exists a fractional brane whose lowest Ramond-Ramond charge is $\mathrm{d}_{3}^{p-3,0}\left(\lambda^{\prime}\right)$, this D-brane is unstable.

The passage from the limit (6.13) with $q=0$ to the actual equivariant K-theory group $\mathrm{K}_{G}^{p}(X)$ requires solving a typically non-trivial extension problem. Even when the spectral sequence collapses at the $\mathrm{E}_{2}$-term, the extension can lead to important torsion corrections which distinguish the classifications of Ramond-Ramond fields based on Bredon cohomology and on equivariant K-theory. The extension problem changes the additive structure on the K-theory group of fluxes from that of the equivariant cohomology classes. This corresponds physically to non-trivial correlations between Ramond-Ramond fields of different degrees, when represented by orbifold differential forms. This torsion enhancement in equivariant K-theory compared to Bredon cohomology can shift the Dirac charge quantization condition on the Ramond-Ramond fields by fractional units and can play an important role near the orbifold points $[12,13]$.

In the non-equivariant case $G=e$, the differential $\mathrm{d}_{3}^{p, 0}$ is known to be given by the Steenrod square cohomology operation $\mathrm{Sq}^{3}$. The vanishing condition $\operatorname{Sq}^{3}(\lambda)=0$ implies the vanishing of the third integer Stieffel-Whitney class of the Poincaré dual cycle to $\lambda \in \mathrm{H}^{p}(X ; \mathbb{Z})$, which is just the condition guaranteeing that the corresponding brane worldvolume is a $\operatorname{spin}^{c}$ submanifold of $X$. Unfortunately, for $G \neq e$ the differential 
$\mathrm{d}_{3}^{p, 0}$ is not known and the geometrical meaning of the condition (6.15) is unclear. It would be interesting to understand this requirement in terms of an obstruction theory for Bredon cohomology, analogously to the non-equivariant case, as this would open up interesting new consistency conditions for D-branes and Ramond-Ramond fields on global orbifolds $[X / G]$. However, we are not aware of any characteristic class theory underlying the Bredon cohomology groups $\mathrm{H}_{G}^{p}(X ; \underline{R}(-))$.

\section{Appendix A. Linear Algebra in Functor Categories}

In this appendix we will summarize some notions about algebra in functor categories that were used in the main text of the paper. They generalize the more commonly used concepts for modules over a ring. For further details see ref. [23].

Let $R$ be a commutative ring, and denote the category of (left) $R$-modules by $R$-Mod. Let $\Gamma$ be a small category, i.e., its class of objects $\operatorname{Obj}(\Gamma)$ is a set. If $\mathcal{C}$ is another category, then one denotes by

$$
[\Gamma, \mathrm{C}]
$$

the functor category of (covariant) functors $\Gamma \rightarrow \mathcal{C}$. The objects of $[\Gamma, \mathrm{C}]$ are (covariant) functors $\phi: \Gamma \rightarrow \mathcal{C}$ and a morphism from $\phi_{1}$ to $\phi_{2}$ is a natural transformation $\alpha: \phi_{1} \rightarrow \phi_{2}$ between functors.

In particular, in the main text we used the functor category

$$
R \Gamma-\operatorname{Mod}:=[\Gamma, R-\operatorname{Mod}]
$$

whose objects are called left $R \Gamma$-modules. If one denotes with $\Gamma^{\mathrm{op}}$ the dual category to $\Gamma$, then there is also the functor category

$$
\operatorname{Mod}-R \Gamma:=\left[\Gamma^{\mathrm{op}}, R-\mathrm{Mod}\right]
$$

of contravariant functors $\Gamma \rightarrow R$-Mod, whose objects are called right $R \Gamma$-modules. As an example, let $G$ be a discrete group regarded as a category with a single object and a morphism for each element of $G$. A covariant functor $G \rightarrow R-$ Mod is then the same thing as a left module over the group ring $R[G]$ of $G$ over $R$.

As the name itself suggests, all standard definitions from the linear algebra of modules have extensions to this more general setting. For instance, the notions of submodule, kernel, cokernel, direct sum, coproduct, etc. can be naturally defined objectwise. If $M$ and $N$ are $R \Gamma$-modules, then $\operatorname{Hom}_{R \Gamma}(M, N)$ is the $R$-module of all natural transformations $M \rightarrow N$. This notation should not be confused with the one used for the set of all morphisms between two objects in $\Gamma$, and usually it is clear from the context.

If $M$ is a right $R \Gamma$-module and $N$ is a left $R \Gamma$-module, then one can define their categorical tensor product

$$
M \otimes_{R \Gamma} N
$$

in the following way. It is the $R$-module given by first forming the direct sum

$$
F=\bigoplus_{\lambda \in \operatorname{Obj}(\Gamma)} M(\lambda) \otimes_{R} N(\lambda)
$$

and then quotienting $F$ by the $R$-submodule generated by all relations of the form

$$
f^{*}(m) \otimes n-m \otimes f_{*}(n)=0,
$$


where $(f: \lambda \rightarrow \rho) \in \operatorname{Mor}(\Gamma), m \in M(\rho), n \in N(\lambda)$ and $f^{*}(m)=M(f)(m), f_{*}(n)=$ $N(f)(n)$. This tensor product commutes with coproducts. If $M$ and $N$ are functors from $\Gamma$ to the category of vector spaces over a field $\mathbb{K}$, then their tensor product is naturally equipped with the structure of a vector space over $\mathbb{K}$. When $\Gamma$ is the orbit category $\operatorname{Or}(G)$ and $R=\mathbb{Z}$, the tensor product has precise limiting cases. For an arbitrary contravariant module $M$ and the constant covariant module $N$, the categorical product $M \otimes_{\mathbb{Z O r}(G)} N$ is the tensor product of the right $\mathbb{Z}[G]$-module $M(G / e)$ with the constant left $\mathbb{Z}[G]$-module $N(G / e), M(G / e) \otimes_{\mathbb{Z}[G]} N(G / e)$. On the other hand, if the contravariant module $M$ is constant and the covariant module $N$ is arbitrary, then $M \otimes_{\mathbb{Z O r}(G)} N$ is just $N(G / G)$.

\section{Appendix B. Equivariant K-Homology}

This appendix is devoted to explaining in more detail some of the definitions and technical constructions in equivariant K-homology theories that were used in the main text to describe states of D-branes in orbifolds.

B.1. Spectral definition. A natural way to define the equivariant homology theory $\mathrm{K}_{\bullet}^{G}$ is by means of a spectrum for equivariant topological K-theory $\mathrm{K}_{G}^{\bullet}$, which within the context of Sect. 2 is a particular covariant functor $\operatorname{Vect}^{G}(-)$ from the orbit category $\operatorname{Or}(G)$ to the tensor category Spec of spectra [20]. Given any $G$-complex $X$, the corresponding pointed $G$-space is $X_{+}=X \amalg$ pt and one defines the loop spectrum $X_{+} \otimes_{G} \underline{\operatorname{Vect}}^{G}(-)$ by

$$
X_{+} \otimes_{G} \underline{\operatorname{Vect}}^{G}(-)=\coprod_{G / H \in \operatorname{Or}(G)}\left(X_{+}^{H} \wedge \underline{\operatorname{Vect}}^{G}(G / H)\right) / \sim,
$$

where the equivalence relation $\sim$ is generated by the identifications $f^{*}(x) \wedge s \sim x \wedge f_{*}(s)$ with $(f: G / K \rightarrow G / H) \in \operatorname{Mor}(\operatorname{Or}(G)), x \in X_{+}^{H}$, and $s \in \underline{\operatorname{Vect}}^{G}(G / K)$.. One then puts

$$
\mathrm{K}_{\bullet}^{G}(X):=\pi_{\bullet}\left(X_{+} \otimes_{G} \underline{\operatorname{Vect}}^{G}(-)\right)
$$

By using various $G$-homotopy equivalences of the loop spectra (B.1), one shows that this definition of equivariant K-homology comes with a natural induction structure in the sense of Sect. 1.2. For the trivial group it reduces to the ordinary $\mathrm{K}$-homology $\mathrm{K}_{\bullet}^{e}=\mathrm{K}$. given by the Bott spectrum $B U$. If $G$ is a finite group, any finite-dimensional representation of $G$ naturally extends to a complex representation of the group ring $\mathbb{C}[G]$. Then there is an analytic assembly map

$$
\text { ass }: \mathrm{K}_{\bullet}^{G}(X) \longrightarrow \mathrm{K}_{\bullet}(\mathbb{C}[G])
$$

to the K-theory of the ring $\mathbb{C}[G]$, induced by the collapsing map $X \rightarrow$ pt and the isomorphisms

$$
\mathrm{K}_{\bullet}(\mathbb{C}[H]) \cong \pi_{\bullet}\left(\underline{\operatorname{Vect}}^{G}(G / H)\right) \cong \mathrm{K}_{\bullet}^{G}(G / H) \cong R(H)
$$

for any subgroup $H \leq G$. In the following we will give two concrete realizations of the homotopy groups (B.2). 
B.2. Analytic definition. The simplest realization of the equivariant K-homology group $\mathrm{K}_{\bullet}^{G}(X)$ is within the framework of an equivariant version of Kasparov's KK-theory $\mathrm{KK}_{\bullet}^{G}$. Let $\mathcal{A}$ be a $G$-algebra, i.e., a $C^{*}$-algebra $\mathcal{A}$ together with a group homomorphism

$$
\lambda: G \longrightarrow \operatorname{Aut}(\mathcal{A}) \text {. }
$$

By a Hilbert $(G, \mathcal{A})$-module we mean a Hilbert $\mathcal{A}$-module $\mathcal{E}$ together with a $G$-action given by a homomorphism $\Lambda: G \rightarrow \operatorname{GL}(\mathcal{E})$ such that

$$
\Lambda_{g}(\varepsilon \cdot a)=\Lambda_{g}\left(\varepsilon \cdot \lambda_{g}(a)\right)
$$

for all $g \in G, \varepsilon \in \mathcal{E}$ and $a \in \mathcal{A}$. Let $\mathcal{L}(\mathcal{E})$ denote the $*$-algebra of $\mathcal{A}$-linear maps $T: \mathcal{E} \rightarrow \mathcal{E}$ admitting an adjoint with respect to the $\mathcal{A}$-valued inner product on $\mathcal{E}$. The induced $G$-action on $\mathcal{L}(\mathcal{E})$ is given by $g \cdot T:=\Lambda_{g} \circ T \circ \Lambda_{g^{-1}}$. Let $\mathcal{K}(\mathcal{E})$ be the subalgebra of $\mathcal{L}(\mathcal{E})$ consisting of generalized compact operators.

Given a pair $(\mathcal{A}, \mathcal{B})$ of $G$-algebras, let $\mathrm{D}^{G}(\mathcal{A}, \mathcal{B})$ be the set of triples $(\mathcal{E}, \phi, T)$, where $\mathcal{E}$ is a countably generated Hilbert $(G, \mathcal{B})$-module, $\phi: \mathcal{A} \rightarrow \mathcal{L}(\mathcal{E})$ is a $*$-homomorphism which commutes with the $G$-action,

$$
\phi\left(\lambda_{g}(a)\right)=\Lambda_{g} \circ \phi(a) \circ \Lambda_{g^{-1}}
$$

for all $g \in G$ and $a \in \mathcal{A}$, and $T \in \mathcal{L}(\mathcal{E})$ such that

1) $[T, \phi(a)] \in \mathcal{K}(\mathcal{E})$ for all $a \in \mathcal{A}$; and

2) $\phi(a)\left(T-T^{*}\right), \phi(a)\left(T^{2}-1\right), \phi(a)(g \cdot T-T) \in \mathcal{K}(\mathcal{E})$ for all $a \in \mathcal{A}$ and $g \in G$.

The standard equivalence relations of KK-theory are now analogously defined. The set of equivalence classes in $\mathrm{D}^{G}(\mathcal{A}, \mathcal{B})$ defines the equivariant $\mathrm{KK}$-theory groups $\operatorname{KK}_{\bullet}^{G}(\mathcal{A}, \mathcal{B})$.

If $X$ is a smooth proper $G$-manifold without boundary, and $G$ acts on $X$ by diffeomorphisms, then the algebra $\mathcal{A}=\mathrm{C}_{0}(X)$ of continuous functions on $X$ vanishing at infinity is a $G$-algebra with automorphism $\lambda_{g}$ on $\mathcal{A}$ given by

$$
\lambda_{g}(f)(x):=\left(g^{*} f\right)(x)=f\left(g^{-1} \cdot x\right),
$$

where $g^{*}$ denotes the pullback of the $G$-action on $X$ by left translation by $g^{-1} \in G$. We define

$$
\mathrm{K}_{\bullet}^{G}(X):=\mathrm{KK}_{\bullet}^{G}\left(\mathrm{C}_{0}(X), \mathbb{C}\right)
$$

with $G$ acting trivially on $\mathbb{C}$. The conditions (B.3) and (B.4) naturally capture the physical requirements that physical orbifold string states are $G$-invariant and also that the worldvolume fields on a fractional D-brane carry a "covariant representation" of the orbifold group [26].

B.3. The equivariant Dirac class. We can determine a canonical class in the abelian group (B.5) as follows. Let $\operatorname{dim}(X)=2 n$, and let $G$ be a finite subgroup of the rotation group $\mathrm{SO}(2 n) .{ }^{11}$ Let

$$
\operatorname{Cliff}(2 n)=\operatorname{Cliff}^{+}(2 n) \oplus \operatorname{Cliff}^{-}(2 n)
$$

\footnotetext{
11 Throughout the extension to $\mathrm{K}_{1}^{G}$ or $\mathrm{K}_{G}^{-1}$ and $\operatorname{dim}(X)$ odd can be described in the same way as in degree zero by replacing $X$ with $X \times \mathbb{S}^{1}$.
} 
denote the complex $\mathbb{Z}_{2}$-graded euclidean Clifford algebra on $n$ generators $e_{1}, \ldots, e_{n}$ with the relations

$$
e_{i} e_{j}+e_{j} e_{i}=-2 \delta_{i j}
$$

A choice of a complete $G$-invariant Riemannian metric on $X$ defines a $G$-bundle of Clifford algebras

$$
\text { Cliff }=\operatorname{Cliff}\left(T_{X}^{*}\right):=\operatorname{Fr}^{*} \times \operatorname{SO}(2 n) \operatorname{Cliff}(2 n)
$$

which is an associated bundle to the metric coframe bundle over $X$, the principal $\mathrm{SO}(2 n)$ bundle $\mathrm{Fr}^{*}=\operatorname{Fr}\left(T_{X}^{*}\right)$ of oriented orthonormal frames on the cotangent bundle $T_{X}^{*}=$ $\mathrm{Fr}^{*} \times \mathrm{SO}(2 n) \mathbb{R}^{2 n}$. The action of $\mathrm{SO}(2 n)$ on the Clifford algebra is through the spin group $\operatorname{Spin}(2 n) \subset \operatorname{Cliff}(2 n)$. The Lie group $\operatorname{Spin}^{c}(2 n) \subset \operatorname{Cliff}(2 n)$ is a central extension of $\mathrm{SO}(2 n)$ by the circle group $\mathrm{U}(1)$,

$$
1 \longrightarrow \mathrm{U}(1) \longrightarrow \operatorname{Spin}^{c}(2 n) \longrightarrow \mathrm{SO}(2 n) \longrightarrow 1
$$

where the quotient map in Eq. (B.6) is consistent with the double covering of $\mathrm{SO}(2 n)$ by $\operatorname{Spin}(2 n)$ so that

$$
\operatorname{Spin}^{c}(2 n)=\operatorname{Spin}(2 n) \times_{\mathbb{Z}_{2}} U(1) .
$$

The $G$-manifold $X$ is said to have a $G$-spinc structure or to be $\mathrm{K}_{G}$-oriented if there is an extension of the coframe bundle to a principal $\operatorname{Spin}^{c}(2 n)$-bundle $\operatorname{Fr}_{L}^{*}$ over $X$ which is compatible with the $G$-action. The extension $\mathrm{Fr}_{L}^{*}$ may be regarded as a principal circle bundle over $\mathrm{Fr}^{*}$,

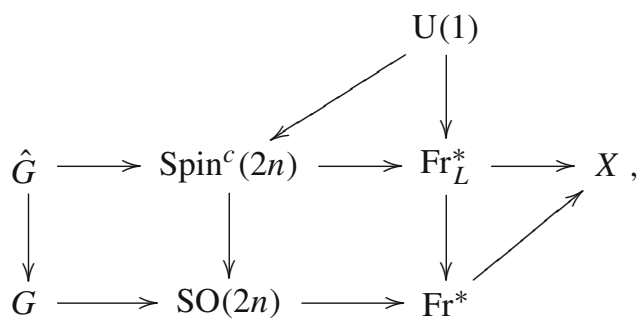

where the pullback square on the bottom left defines the required covering of the orbifold group $G<\operatorname{SO}(2 n)$ by a subgroup of the $\operatorname{spin}^{c}$ group $\hat{G}<\operatorname{Spin}^{c}(2 n)$. This lift is also necessary in order to account for the spacetime fermions present in string theory. The kernel of the homomorphism $\hat{G} \rightarrow G$ is identified with the circle group $\mathrm{U}(1)<\operatorname{Spin}^{c}(2 n)$ in the Clifford algebra Cliff $(2 n)$. We fix a choice of lift and hence assume that $G$ is a discrete subgroup of the $\operatorname{spin}^{c}$ group. $\mathbb{Z}_{2}$-graded Clifford modules are likewise extended to representations of $\mathbb{C}[G] \otimes \operatorname{Cliff}(2 n)$, with $\mathbb{C}[G]$ the group ring of $G$, called $G$-Clifford modules. The topological obstruction to the existence of a $G$-spin ${ }^{c}$ structure on $X$ is the equivariant third integral Stiefel-Whitney class $\left(W_{3}\right)_{G}\left(T_{X}^{*}\right) \in \mathrm{H}_{G}^{3}(X ; \mathbb{Z})$ of the cotangent bundle $T_{X}^{*}$ in Borel cohomology.

The associated bundles of half-spinors on $X$ are defined as

$$
S^{ \pm}=S\left(T_{X}^{*}\right)^{ \pm}:=\operatorname{Fr}_{L}^{*} \times \operatorname{Spin}^{c}(2 n) \Delta^{ \pm},
$$

where $\Delta^{ \pm}$are the irreducible half-spin representations of $\operatorname{SO}(2 n)$. Since $G$ lifts to $\hat{G}$ in the $\operatorname{spin}^{c}$ group, the half-spin representations $\Delta^{ \pm}$restrict to representations of $G$ and 
the half-spinor bundles (B.7) are $G$-bundles. The $G$-invariant Levi-Civita connection determines a connection one-form on $\mathrm{Fr}^{*}$, and together with a choice of $G$-invariant connection one-form on the principal $\mathrm{U}(1)$-bundle $\mathrm{Fr}_{L}^{*} \rightarrow \mathrm{Fr}^{*}$, they determine a connection one-form on the principal $\operatorname{Spin}^{c}(2 n)$-bundle $\operatorname{Fr}_{L}^{*} \rightarrow X$ which is $G$-invariant. This determines an invariant connection

$$
\nabla^{S \otimes E}:=\nabla^{S} \otimes 1+1 \otimes \nabla^{E}: C^{\infty}\left(X, S^{+} \otimes E\right) \longrightarrow C^{\infty}\left(X, T_{X}^{*} \otimes S^{+} \otimes E\right),
$$

where $\nabla^{E}$ is a $G$-invariant connection on a $G$-bundle $E \rightarrow X$. The contraction given by Clifford multiplication defines a map

$$
\mathrm{C} \ell: \mathrm{C}^{\infty}\left(X, T_{X}^{*} \otimes S^{+} \otimes E\right) \longrightarrow \mathrm{C}^{\infty}\left(X, S^{-} \otimes E\right)
$$

which graded commutes with the $G$-action, and the $G$-invariant $\operatorname{spin}^{c}$ Dirac operator on $X$ with coefficients in $E$ is defined as the composition

$$
\not D_{E}^{X}=\mathrm{C} \ell \circ \nabla^{S \otimes E} \text {. }
$$

We will view the operator (B.8) as an operator on $\mathrm{L}^{2}$-spaces

$$
\not D_{E}^{X}: \mathrm{L}^{2}\left(X, S^{+} \otimes E\right) \longrightarrow \mathrm{L}^{2}\left(X, S^{-} \otimes E\right) \text {. }
$$

It induces a class $\left[D_{E}^{X}\right] \in \mathrm{K}_{0}^{G}(X)$ as follows. The $G$-algebra $\mathrm{C}_{0}(X)$ acts on the $\mathbb{Z}_{2^{-}}$ graded $G$-Hilbert space $\mathcal{E}:=\mathrm{L}^{2}(X, S \otimes E)$ by multiplication. Define the bounded $G$-invariant operator $T:=D_{E}^{X}\left(\left(\not D_{E}^{X}\right)^{2}+1\right)^{-1 / 2} \in \operatorname{Fred}_{G}$. Then $\left[D_{E}^{X}\right]$ is represented by the $G$-equivariant Fredholm module $(\mathcal{E}, T)$.

B.4. Geometric definition. The natural geometric description of D-branes in an orbifold space is provided by the topological version of the groups $\mathrm{K}_{\bullet}^{G}(X)$ due to Baum, Connes and Douglas $[8,7]$. This can be defined for an arbitrary discrete, countable group $G$ on the category of proper, finite $G$-complexes $X$ and proven to be isomorphic to analytic equivariant K-homology [10]. Recall that the topological equivariant K-theory $\mathrm{K}_{G}^{\bullet}(X)$ is defined by applying the Grothendieck functor $\mathrm{K}^{\bullet}$ to the additive category $\operatorname{Vect}_{G}^{\mathbb{C}}(X)$ whose objects are complex $G$-vector bundles over $X$, i.e., $\mathrm{K}_{G}^{\bullet}(X):=\mathrm{K}^{\bullet}\left(\operatorname{Vect}_{G}^{\mathbb{C}}(X)\right)$. In the homological setting, the relevant category is instead the additive category of $G$-equivariant $K$-cycles $\mathrm{D}^{G}(X)$, whose objects are triples $(W, E, f)$, where

(a) $W$ is a manifold without boundary with a smooth proper cocompact $G$-action and $G$-spin ${ }^{c}$ structure;

(b) $E$ is an object in $\operatorname{Vect}_{G}^{\mathbb{C}}(W)$; and

(c) $f: W \rightarrow X$ is a $G$-map.

Two $G$-equivariant K-cycles $(W, E, f)$ and $\left(W^{\prime}, E^{\prime}, f^{\prime}\right)$ are said to be isomorphic if there is a $G$-equivariant diffeomorphism $h: W \rightarrow W^{\prime}$ preserving the $G$-spin ${ }^{c}$ structures on $W, W^{\prime}$ such that $h^{*}\left(E^{\prime}\right) \cong E$ and $f^{\prime} \circ h=f$.

Define an equivalence relation $\sim$ on the category $\mathrm{D}^{G}(X)$ generated by the operations of 
- Bordism: $\left(W_{i}, E_{i}, f_{i}\right) \in \mathrm{D}^{G}(X), i=0,1$ are bordant if there is a triple $(M, E, f)$ where $M$ is a manifold with boundary $\partial M$, with a smooth proper cocompact $G$-action and $G$-spin ${ }^{c}$ structure, $E \rightarrow M$ is a complex $G$-vector bundle, and $f: M \rightarrow X$ is a $G$-map such that $\left(\partial M,\left.E\right|_{\partial M},\left.f\right|_{\partial M}\right) \cong\left(W_{0}, E_{0}, f_{0}\right) \amalg\left(-W_{1}, E_{1}, f_{1}\right)$. Here $-W_{1}$ denotes $W_{1}$ with the reversed $G$-spin ${ }^{c}$ structure;

- Direct sum: If $(W, E, f) \in \mathrm{D}^{G}(X)$ and $E=E_{0} \oplus E_{1}$, then

$$
(W, E, f) \cong\left(W, E_{0}, f\right) \amalg\left(W, E_{1}, f\right) ;
$$

and

- Vector bundle modification: Let $(W, E, f) \in \mathrm{D}^{G}(X)$ and $H$ be an even-dimensional $G$-spin ${ }^{c}$ vector bundle over $W$. Let $\widehat{W}=\mathbb{S}(H \oplus \mathbb{1})$ denote the sphere bundle of $H \oplus \mathbb{1}$, which is canonically a $G$-spin ${ }^{c}$ manifold, with $G$-bundle projection $\pi: \widehat{W} \rightarrow W$. Let

$$
S(H)=S(H)^{+} \oplus S(H)^{-}
$$

denote the $\mathbb{Z}_{2}$-graded $G$-bundle over $W$ of spinors on $H$. Set $\widehat{E}=\pi^{*}\left(\left(S(H)^{+}\right)^{\vee} \otimes E\right)$ and $\widehat{f}=f \circ \pi$. Then $(\widehat{W}, \widehat{E}, \widehat{f}) \in \mathrm{D}^{G}(X)$ is the vector bundle modification of $(W, E, f)$ by $H$.

We set

$$
\mathrm{K}_{0,1}^{G}(X)=\mathrm{D}_{\text {even,odd }}^{G}(X) / \sim,
$$

where the parity refers to the dimension of the K-cycle, which is preserved by $\sim$.

Using the equivariant Dirac class, one can construct a homomorphism from the geometric to the analytic K-homology group. On K-cycles we define $(W, E, f) \mapsto f_{*}\left[D_{E}^{W}\right]$ and extend linearly. This map can be used to express $G$-index theorems within this homological framework and it extends to give an isomorphism between the two equivariant K-homology groups [10]. (See also ref. [57] for a related construction in the non-equivariant case.)

\section{Appendix C. D-Brane charges of Equivariant K-Cycles}

In this appendix we will review the construction of the equivariant Gysin homomorphism and how it shows that D-brane charges on the orbifold $[X / G]$ take values in the equivariant K-theory $\mathrm{K}_{G}^{\bullet}(X)$. Let $X$ and $W$ be smooth compact $G$-manifolds, and $f: W \rightarrow X$ a smooth proper $G$-map. We begin by dealing with the non-equivariant setting $G=e$. Assume that the $\mathbb{Z}_{2}$-graded bundle $v$ of Eq. (4.10) is of even rank $r=2 n$ and endowed with a $\operatorname{spin}^{c}$ structure. We will generalize the construction $[49,54,62]$, establishing that the charge of a D-brane supported on $W$ with Chan-Paton gauge bundle $E \rightarrow W$ in Type II superstring theory without $H$-flux takes values in the complex K-theory of spacetime $X$, to D-branes represented by generic topological $\mathrm{K}$-cycles $(W, E, f)$, i.e., including those D-branes which are not representable as wrapping embedded cycles in $X$. It is based on the diagram

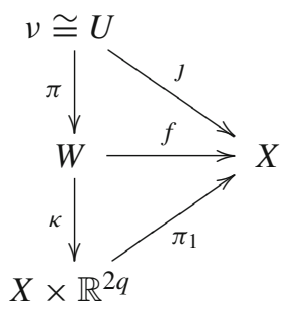


over the brane immersion $f$, which we explain momentarily. The $\operatorname{spin}^{c}$ condition on the bundle $v$ is the appropriate generalization of the Freed-Witten anomaly cancellation condition [30] to this situation. It amounts to a choice of line bundle $L \rightarrow W$ whose first Chern class

$$
c_{1}(L) \in \mathrm{H}^{2}(W ; \mathbb{Z})
$$

obeys $c_{1}(L) \equiv f^{*} w_{2}\left(T_{X}\right)-w_{2}\left(T_{W}\right) \bmod 2$, where $w_{2}\left(T_{X}\right)$ and $w_{2}\left(T_{W}\right)$ are the second Stiefel-Whitney classes of the tangent bundles of $X$ and $W$. The set of all such $\mathrm{K}$-orientations is an affine space modelled on $2 \mathrm{H}^{2}(W ; \mathbb{Z})$.

Consider first the usual case where $f: W \hookrightarrow X$ is a smoothly embedded cycle. Then the virtual bundle $v$ can be identified (in KO-theory) with the normal bundle to $W$ with respect to $f$, which is the quotient bundle $\pi: f^{*}\left(T_{X}\right) / T_{W} \rightarrow W$. Upon choosing a Riemannian metric on $X$, we can identify $v$ with a tubular neighbourhood $U$ of $f(W)$ via a diffeomorphism from the open embedding $J: U \hookrightarrow X$ onto a neighbourhood of the zero section embedding $W \hookrightarrow v$. Let $\left[\pi^{*} S(v)^{+}, \pi^{*} S(v)^{-} ; c(v)\right]$ be the AtiyahBott-Shapiro representative of the Thom class Thom $(v)$, in the K-theory with compact vertical support

$$
\mathrm{K}_{\mathrm{cpt}}^{r}(v):=\mathrm{K}^{r}(v, v \backslash W)
$$

which restricts to the Bott class $u^{-n} \in \mathrm{K}^{-r}$ (pt) on each fibre of $v$. Here

$$
S(v)^{ \pm} \longrightarrow W
$$

are the half-spinor bundles associated to $v$ and the morphism $c(v): \pi^{*} S(v)^{+} \rightarrow$ $\pi^{*} S(v)^{-}$is given by Clifford multiplication by the tautological section $v$ of the bundle $\pi^{*} v \rightarrow v$ which assigns to a vector in $v$ the same vector in $\pi^{*} v$.

Then one can define the Gysin homomorphism in ordinary K-theory

$$
f_{!}^{\mathrm{K}}: \mathrm{K}^{\bullet}(W) \longrightarrow \mathrm{K}^{\bullet}(X) \text {. }
$$

It is defined as the composition of the Thom isomorphism

$$
\begin{aligned}
\mathrm{K}^{\bullet}(W) & \stackrel{\approx}{\rightarrow} \mathrm{K}_{\mathrm{cpt}}^{\bullet}(v) \\
\xi & \longmapsto \pi^{*}(\xi) \otimes \operatorname{Thom}(v)
\end{aligned}
$$

with the natural "extension by zero" homomorphism $J: \mathrm{K}_{\mathrm{cpt}}^{\bullet}(v) \rightarrow \mathrm{K}^{\bullet}(X)$ given by composing $\mathrm{K}^{\bullet}(U, U \backslash W) \rightarrow \mathrm{K}^{\bullet}(X, X \backslash W) \rightarrow \mathrm{K}^{\bullet}(X)$, where the first map is the excision isomorphism and the second map is induced by the inclusion $(X, \mathrm{pt}) \hookrightarrow(X, W)$. For a general smooth proper map $f: W \rightarrow X$, we use the fact that every smooth compact manifold $W$ can be smoothly embedded in $\mathbb{R}^{2 q}$ for $q$ sufficiently large to define a parametrized version that yields an embedding

$$
\kappa: W \longrightarrow X \times \mathbb{R}^{2 q},
$$

whose normal bundle is $\operatorname{spin}^{c}$. The corresponding Gysin map is a homomorphism

$$
\kappa_{!}^{\mathrm{K}}: \mathrm{K}^{\bullet}(W) \longrightarrow \mathrm{K}_{\mathrm{cpt}}^{\bullet}\left(X \times \mathbb{R}^{2 q}\right) \text {. }
$$


The Gysin homomorphism $f_{!}^{\mathrm{K}}: \mathrm{K}^{\bullet}(W) \rightarrow \mathrm{K}^{\bullet}(X)$ is then defined as the composition of $\kappa_{!}^{\mathrm{K}}$ with the inverse Thom isomorphism $\mathrm{K}_{\mathrm{cpt}}^{\bullet}\left(X \times \mathbb{R}^{2 q}\right) \cong \mathrm{K}^{\bullet}(X)$ for the trivial $\operatorname{spin}^{c}$ bundle

$$
\pi_{1}: X \times \mathbb{R}^{2 q} \longrightarrow X .
$$

By homotopy invariance of K-theory and functoriality for pushforward maps, the map $f_{!}^{\mathrm{K}}$ is independent of the choice of identification of the normal bundle with a tubular neighbourhood and of Whitney embedding $W \hookrightarrow \mathbb{R}^{2 q}$.

Let us now consider the $G$-actions on $W$ and on $X$. In a similar way as in ordinary $\mathrm{K}$-theory, if $v$ is $\mathrm{K}_{G}$-oriented then one has the equivariant Thom isomorphism

$$
\begin{aligned}
\mathrm{K}_{G}^{\bullet}(W) & \stackrel{\approx}{\rightarrow} \mathrm{K}_{G, \mathrm{cpt}}^{\bullet}(v) \\
\xi & \longmapsto \pi^{*}(\xi) \otimes \operatorname{Thom}_{G}(v),
\end{aligned}
$$

where the equivariant Thom class $\operatorname{Thom}_{G}(\nu) \in \mathrm{K}_{G \text {,cpt }}^{r}(\nu)$ is defined in the same way as above using the $G$-spin ${ }^{c}$ structure on $v$ and the equivariant version of the AtiyahBott-Shapiro construction [42]. The associated Gysin homomorphism, constructed as above via a choice of $G$-invariant Riemannian metric on $X$ and of $G$-invariant Whitney embedding $W \hookrightarrow \mathbb{R}^{2 q}$ with $G$ acting trivially on $\mathbb{R}^{2 q}$, is the pushforward map $f_{!}^{\mathrm{K}_{G}}: \mathrm{K}_{G}^{\bullet}(W) \rightarrow \mathrm{K}_{G}^{\bullet}(X)$. This establishes that the charge of a fractional D-brane in the Type II spacetime orbifold $[X / G]$, associated to a generic $G$-equivariant K-cycle $(W, E, f) \in \mathrm{D}^{G}(X)$ on the covering space $X$, takes values $f_{!}^{\mathrm{K}_{G}}([E]) \in \mathrm{K}_{G}^{\bullet}(X)$ in the equivariant $\mathrm{K}$-theory of $X$.

Acknowlegements. We are grateful to J. Figueroa-O'Farrill, D. Freed, J. Greenlees, J. Howie, A. Konechny, W. Lück, M. Lawson, R. Reis, P. Turner and S. Willerton for helpful discussions and correspondence. This work was supported in part by the Marie Curie Research Training Network Grant ForcesUniverse (contract no. MRTN-CT-2004-005104) from the European Community's Sixth Framework Programme. The work of A.V. was supported by the School of Mathematical and Computer Sciences at Heriot-Watt University, and in part by the German Research Foundation (Deutsche Forschungsgemeinschaft (DFG)) through the Institutional Strategy of the University of Göttingen.

\section{References}

1. Adem, A., Ruan, Y.: Twisted orbifold K-theory. Commun. Math. Phys. 237, 533-556 (2003)

2. Asakawa, T., Sugimoto, S., Terashima, S.: D-branes, matrix theory and K-homology. J. High Energy Phys. 0203, 034 (2002)

3. Atiyah, M.F.: K-Theory NewYork: Benjamin, 1967

4. Atiyah, M.F., Segal, G.B.: Equivariant K-theory and completion. J. Diff. Geom. 3, 1-18 (1969)

5. Atiyah, M.F., Segal, G.B.: On equivariant Euler characteristics. J. Geom. Phys. 6, 399-405 (1989)

6. Baum, P., Connes, A.: Chern character for discrete groups. In: A Fête of Topology, Amsterdam: North Holland, 1987, pp. 163-232

7. Baum, P., Connes, A.: Geometric K-theory for Lie groups and foliations. Enseign. Math. 46, 3-42 (2000)

8. Baum, P., Douglas, R.G.: K-homology and index theory. Proc. Symp. Pure Math. 38, 117-173 (1982)

9. Baum, P., Brylinksi, J.-L., MacPherson, R.: Cohomologie equivariante délocalisée. C. R. Acad. Sci. Paris Ser. I Math. 300, 605-608 (1985)

10. Baum, P., Higson, N., Schick, T.: On the equivalence of geometric and analytic K-homology. Pure Appl. Math. Quart. 3, 1-24 (2007)

11. Belov, D.M., Moore, G.W.: Type II actions from 11-dimensional Chern-Simons theories. Preprint. http:// arxiv.org/abs/hep-th/0611020v1, 2006

12. Bergman, O., Gimon, E.G., Kol, B.: Strings on orbifold lines. J. High Energy Phys. 0105, 019 (2001) 
13. Bergman, O., Gimon, E.G., Sugimoto, S.: Orientifolds, RR torsion and K-theory. J. High Energy Phys. 0105, 047 (2001)

14. de Boer, J., Dijkgraaf, R., Hori, K., Keurentjes, A., Morgan, J., Morrison, D.R., Sethi, S.: Triples, fluxes and strings. Adv. Theor. Math. Phys. 4, 995-1186 (2002)

15. Block, J., Getzler, E.: Equivariant cyclic cohomology and equivariant differential forms. Ann. Sci. ENS 27, 493-527 (1994)

16. Bredon, G.E.: Equivariant Cohomology Theories. Berlin-Heidelberg-NewYork: Springer, 1967

17. Bunke, U.: Orbifold index and equivariant K-homology. Math. Ann. 339, 175-194 (2007)

18. Carey, A.L., Mickelsson, J., Wang, B.-L.: Differential twisted K-theory and applications. Preprint. http:// arxiv:0708.3114v2[math.KT], 2007

19. Cheung, Y.-K.E., Yin, Z.: Anomalies, branes and currents. Nucl. Phys. B 517, 69-91 (1998)

20. Davis, J.F., Lück, W.: Spaces over a category and assembly maps in isomorphism conjectures in K- and L-theory. K-Theory 15, 201-252 (1998)

21. Diaconescu, D.-E., Gomis, J.: Fractional branes and boundary states in orbifold theories. J. High Energy Phys. 0010, 001 (2000)

22. Diaconescu, D.-E., Moore, G.W., Witten, E.: $E_{8}$ gauge theory and a derivation of K-theory from M-theory. Adv. Theor. Math. Phys. 6, 1031-1134 (2003)

23. tom Dieck, T.: Transformation Groups. Berlin: Walter de Gruyter, 1987

24. Dixon, L.J., Harvey, J.A., Vafa, C., Witten, E.: Strings on orbifolds. Nucl. Phys. B 261, 678-686 (1985)

25. Dixon, L.J., Harvey, J.A., Vafa, C., Witten, E.: Strings on orbifolds 2. Nucl. Phys. B 274, 285-314 (1986)

26. Douglas, M.R., Moore, G.W.: D-branes, quivers and ALE instantons. Preprint. http://arxiv:hep-th/ 9603167v1, 1996

27. Douglas, M.R., Greene, B.R., Morrison, D.R.: Orbifold resolution by D-branes. Nucl. Phys. B 506, 84-106 (1997)

28. Freed, D.S.: Dirac charge quantization and generalized differential cohomology. Surv. Diff. Geom. VII, 129-194 (2000)

29. Freed, D.S., Hopkins, M.J.: On Ramond-Ramond fields and K-theory. J. High Energy Phys. 0005, 044 (2000)

30. Freed, D.S., Witten, E.: Anomalies in string theory with D-branes. Asian J. Math. 3, 819-851 (1999)

31. Freed, D.S., Hopkins, M.J., Teleman, C.: Twisted equivariant K-theory with complex coefficients. Preprint. http://arxiv:math/0206257v4, 2002

32. Freed, D.S., Moore, G.W., Segal, G.B.: The uncertainty of fluxes. Commun. Math. Phys. 271, 247-274 (2007)

33. Freed, D.S., Moore, G.W., Segal, G.B.: Heisenberg groups and noncommutative fluxes. Ann. Phys. 322, 236-285 (2007)

34. García-Compeán, H.: D-branes in orbifold singularities and equivariant K-theory. Nucl. Phys. B 557, 480-504 (1999)

35. Green, M.B., Harvey, J.A., Moore, G.W.: I-brane inflow and anomalous couplings on D-branes. Class. Quant. Grav. 14, 47-52 (1997)

36. Harvey, J.A., Moore, G.W.: Noncommutative tachyons and K-theory. J. Math. Phys. 42, 2765-2780 (2001)

37. Hopkins, M.J., Singer, I.M.: Quadratic functions in geometry, topology and M-theory. J. Diff. Geom. 70, 329-452 (2005)

38. Illman, S.: Smooth equivariant triangulations of $G$-manifolds for $G$ a finite group. Math. Ann. 233, 199-220 (1978)

39. Illman, S.: The equivariant triangulation theorem for actions of compact Lie groups. Math. Ann. 262, 487-501 (1983)

40. Karoubi, M.: Equivariant K-theory of real vector spaces and real projective spaces. Topol. Appl. 122, 531-546 (2002)

41. Kriz, I., Pando Zayas, L.A., Quiroz, N.: Comments on D-branes on orbifolds and K-theory. Int. J. Mod. Phys. A23, 933-974 (2008)

42. Landweber, G.D.: Representation rings of Lie superalgebras. K-Theory 36, 115-168 (2005)

43. Lott, J.: $\mathbb{R} / \mathbb{Z}$ index theory. Comm. Anal. Geom. 2, 279-311 (1994)

44. Lück, W.: Chern characters for proper equivariant homology theories and applications to K- and L-theory. J. Reine Angew. Math. 543, 193-234 (2002)

45. Lück, W.: Equivariant cohomological Chern characters. Int. J. Alg. Comp. 15, 1025-1052 (2006)

46. Lück, W., Oliver, B.: Chern characters for the equivariant K-theory of proper $G$-CW-complexes. Progr. Math. 196, 217-248 (2001)

47. Lück, W., Oliver, B.: The completion theorem in K-theory for proper actions of a discrete group. Topology 40, 585-616 (2001)

48. Maldacena, J.M., Moore, G.W., Seiberg, N.: D-brane instantons and K-theory charges. J. High Energy Phys. 0111, 062 (2001) 
49. Minasian, R., Moore, G.W.: K-theory and Ramond-Ramond charge. J. High Energy Phys. 9711, 002 (1997)

50. Mislin, G., Valette, A.: Proper Group Actions and the Baum-Connes Conjecture. Basel-Boston: Birkhäuser Verlag, 2003

51. Moore, G.W., Parnachev, A.: Localized tachyons and the quantum McKay correspondence. J. High Energy Phys. 0411, 086 (2004)

52. Moore, G.W., Witten, E.: Self-duality, Ramond-Ramond fields and K-theory. J. High Energy Phys. 0005, 032 (2000)

53. Nori, M.V.: The Hirzebruch-Riemann-Roch theorem. Michigan Math. J. 48, 473-482 (2000)

54. Olsen, K., Szabo, R.J.: Constructing D-branes from K-theory. Adv. Theor. Math. Phys. 4, 889-1025 (2000)

55. Periwal, V.: D-brane charges and K-homology. J. High Energy Phys. 0007, 041 (2000)

56. Reis, R.M.G., Szabo, R.J.: Geometric K-homology of flat D-branes. Commun. Math. Phys. 266, 71-122 (2006)

57. Reis, R.M.G., Szabo, R.J., Valentino, A.: KO-homology and type I string theory. Rev. Math. Phys. 21, 1091-1143 (2009)

58. Segal, G.B.: Equivariant K-theory. Publ. Math. IHES 34, 129-151 (1968)

59. Słomińska, J.: On the equivariant Chern character homomorphism. Bull. Acad. Pol. Sci. 24, 909-913 (1976)

60. Szabo, R.J.: D-branes, tachyons and K-homology. Mod. Phys. Lett. A 17, 2297-2316 (2002)

61. Thomasson, R.W.: Une formule de Lefschetz en K-theories equivariante algebraique. Duke Math. J. 68, 447-462 (1992)

62. Witten, E.: D-branes and K-theory. J. High Energy Phys. 9812, 019 (1998)

Communicated by N.A. Nekrasov 